U.S. DEPARTMENT OF COMMERCE / National Bureau of Standards

\title{
Interactive FORTRAN Programs for Micro Computers to Calculate the Thermophysical Properties of Twelve Fluids [MIPROPS]
}

Robert D. McCarty 

basis for the nation's physical measurement system, (2) scientific and technological services for industry and government, (3) a technical basis for equity in trade, and (4) technical services to promote public safety. The Bureau's technical work is performed by the National Measurement Laboratory, the National Engineering Laboratory, the Institute for Computer Sciences and Technology, and the Institute for Materials Science and Engineering.

\section{The National Measurement Laboratory}

Provides the national system of physical and chemical measurement; coordinates the system with measurement systems of other nations and furnishes essential services leading to accurate and uniform physical and chemical measurement throughout the Nation's scientific community, industry, and commerce; provides advisory and research services to other Government agencies; conducts physical and chemical research; develops, produces, and distributes Standard Reference Materials; and provides calibration services. The Laboratory consists of the following centers:
- Basic Standards ${ }^{2}$

- Radiation Research

- Chemical Physics

- Analytical Chemistry

\section{The National Engineering Laboratory}

Provides technology and technical services to the public and private sectors to address national needs and to solve national problems; conducts research in engineering and applied science in support of these efforts; builds and maintains competence in the necessary disciplines required to carry out this research and technical service; develops engineering data and measurement capabilities; provides engineering measurement traceability services; develops test methods and proposes engineering standards and code changes; develops and proposes new engineering practices; and develops and improves mechanisms to transfer results of its research to the ultimate user. The Laboratory consists of the following centers:
- Applied Mathematics

- Electronics and Electrical Engineering ${ }^{2}$

- Manufacturing Engineering

- Building Technology

- Fire Research

- Chemical Engineering ${ }^{2}$

\section{The Institute for Computer Sciences and Technology}

Conducts research and provides scientific and technical services to aid Federal agencies in the selection, acquisition, application, and use of computer technology to improve effectiveness and economy in Government operations in accordance with Public Law 89-306 (40 U.S.C. 759), relevant Executive Orders, and other directives; carries out this mission by managing the Federal Information Processing Standards Program, developing Federal ADP standards guidelines, and managing Federal participation in ADP voluntary standardization activities; provides scientific and technological advisory services and assistance to Federal agencies; and provides the technical foundation for computer-related policies of the Federal Government. The Institute consists of the following centers:

\author{
- Programming Science and \\ Technology \\ - Computer Systems \\ Engineering
}

\section{The Institute for Materials Science and Engineering}

Conducts research and provides measurements, data, standards, reference materials, quantitative understanding and other technical information funda. mental to the processing, structure, properties and performance of materials addresses the scientific basis for new advanced materials technologies; plans research around cross-country scientific themes such as nondestructive evaluation and phase diagram development; oversees Bureau-wide technical programs in nuclear reactor radiation research and nondestructive evaluation; and broadly disseminates generic technical information resulting from its programs. The Institute consists of the following Divisions:
- Ceramics

- Fracture and Deformation ${ }^{3}$

- Polymers

- Metallurgy

- Reactor Radiation 


\section{Interactive FORTRAN Programs for Micro Computers to Calculate the Thermophysical Properties of Twelve Fluids [MIPROPS]}

\section{Robert D. McCarty}

Thermophysics Division

Center for Chemical Engineering

National Engineering Laboratory

National Bureau of Standards

Boulder, Colorado 80303

Sponsored in part by

National Aeronautics and Space Administration

Lyndon B. Johnson Space Center

Houston, Texas 77058

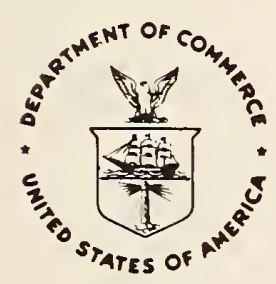

U.S. DEPARTMENT OF COMMERCE, Malcolm Baldrige, Secretary

NATIONAL BUREAU OF STANDARDS, Ernest Ambler, Director 
National Bureau of Standards Technical Note 1097

Natl. Bur. Stand. (U.S.), Tech Note 1097, 90 pages (May 1986)

CODEN: NBTNAE

\section{U.S. GOVERNMENT PRINTING OFFICE \\ WASHINGTON: 1986}

For sale by the Superintendent of Documents, U.S. Government Printing Office, Washington, DC 20402 


\section{CONTENTS}

Page

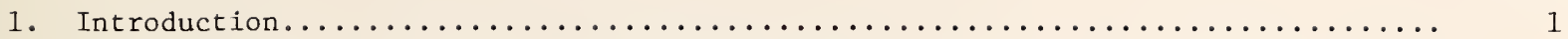

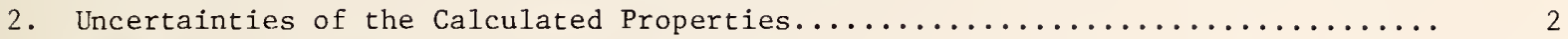

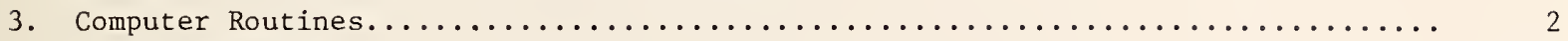

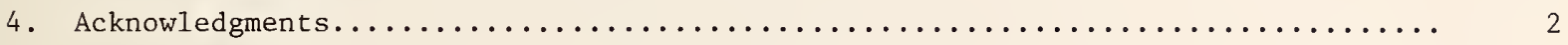

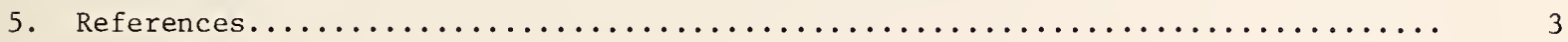

Appendix A. Description of Individual Fluids........................

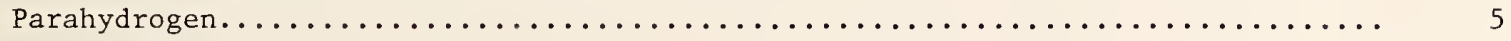

Helium. $\ldots \ldots \ldots \ldots \ldots \ldots \ldots \ldots \ldots \ldots \ldots \ldots \ldots \ldots \ldots \ldots \ldots \ldots \ldots \ldots \ldots \ldots \ldots \ldots \ldots$

Nitrogen $\ldots \ldots \ldots \ldots \ldots \ldots \ldots \ldots \ldots \ldots \ldots \ldots \ldots \ldots \ldots \ldots \ldots \ldots \ldots \ldots \ldots \ldots \ldots \ldots \ldots \ldots \ldots$

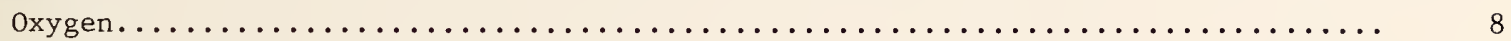

Argon $\ldots \ldots \ldots \ldots \ldots \ldots \ldots \ldots \ldots \ldots \ldots \ldots \ldots \ldots \ldots \ldots \ldots \ldots \ldots \ldots \ldots \ldots \ldots \ldots \ldots \ldots$

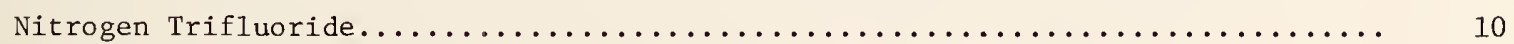

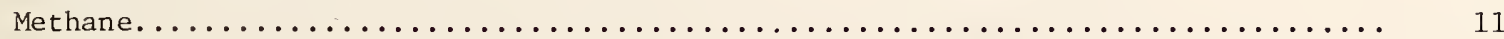

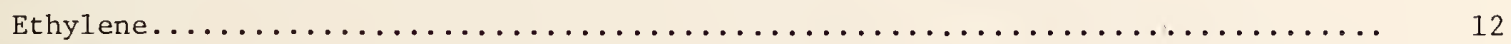

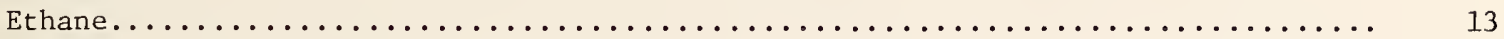

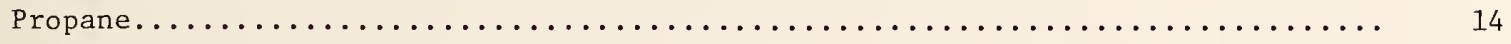

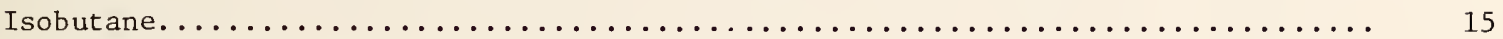

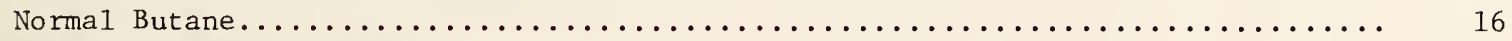

Appendix B. Sample Computer Sessions..............................

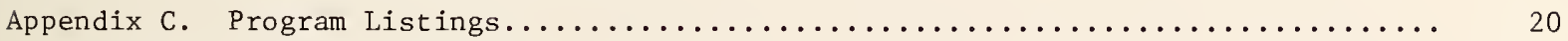

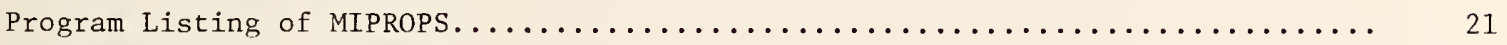

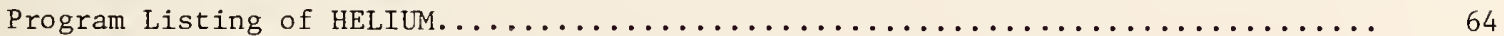



INTERACTIVE FORTRAN PROGRAMS FOR MICRO COMPUTERS TO CALCULATE THE

THERMOPHYSICAL PROPERTIES OF TWELVE FLUIDS [MIPROPS]

\author{
Robert D. McCarty \\ Thermophysics Division \\ National Engineering Laboratory \\ National Bureau of Standards \\ Boulder, Colorado 80303
}

The thermophysical and transport properties of selected fluids have been programmed in FORTRAN 77 which is available for micro computers. The input variables are any two of $\mathrm{P}, \rho, \mathrm{T}$ (pressure, density, and temperature) in the single phase regions, and either $\mathrm{P}$ or $\mathrm{T}$ for the saturated liquid or vapor states. The output is pressure, density, temperature, internal energy, enthalpy, entropy, specific heat capacities $\left(C_{p}\right.$ and $\left.C_{v}\right)$, speed of sound and, in most cases, viscosity, thermal conductivity and dielectric constant.

The fluids included are: helium, hydrogen, nitrogen, oxygen, argon, nitrogen trifluoride, methane, ethylene, ethane, propane, iso- and normal butane. The programs give properties in both the liquid and vapor states over a wide range of temperature and pressure. Copies of the program may be obtained from the office of Standard Reference Data, Room A320, Physics Building, National Bureau of Standards, Gaithersburg, MD 20899.

Key words: argon; computer programs; density; dielectric constant; enthalpy; entropy; equation of state; ethane, ethylene; heat capacity; helium; hydrogen; internal energy; isobutane; methane; nitrogen; nitrogen trifluoride; nornal butane; oxygen; pressure; speed of sound; temperature; thermal conductivity; viscosity.

\title{
1. Introduction
}

Recent technological advances in the field of personal computers have made it possible and practical to run large FORTRAN programs on these machines. The programs presented here will run satisfactorily on a wide variety of personal computers on the market today.

The programs are a direct translation of those developed by Younglove [1,2], with five additional fluids added [3]. The separate program for helium originally appeared in NBS Technical Note 1025 by McCarty [4] and was, in turn, derived from an earlier evaluation of helium data [5]. The programs in the above-mentioned documents were written as interactive FORTRAN IV programs intended to run on large main frames. The programs presented here are translations of the originals to FURTRAN 77 adapted to personal computers and reproduce the calculations of the originals exactly.

All of the fluids except helium utilize the same mathematical model of the equation of state which was originally proposed by Jacobsen [6]. The model for the heliurn thermodynamic surface is of a different form, and a separate progran was necessary for that fluid. The mathematical model of the transport properties of the fluids which have been added to those from NBS Technical Note 1048, has been improved and new routines are utilized for those fluids. 
The programs produce the transport properties for all of the fluids except hydrogen, nitrogen trifluoride, ethylene, ethane and propane. The dielectric constant is also provided for all of the fluids except argon, ethylene and nitrogen trifluoride.

There is a section devoted to each fluid in Appendix A and the reader is referred to those sections for further information on an individual fluid. Listings of the source code and sample calculations may be found in Appendices $B$ and $C$.

\section{Uncertainties of the Calculated Properties}

When calculating thermodynamic properties from an empirical equation of state, one should be aware of certain problem areas of the thermodynamic surface. In the critical region $\left(\rho_{c} \pm 0.2 \rho_{c}\right.$ and $\left.\mathrm{T}_{\mathrm{C}} \pm 0.05 \mathrm{~T}_{\mathrm{C}}\right)$, the calculated density may be in error by several percent, while the calculated pressure or temperature will not be as inaccurate. Values of specific heat capacity and thermal conductivity in the critical region become very large and no realistic estimate of the uncertainty may be made. Saturation boundaries, gas-liquid, and liquid-solid are potential areas of large uncertainties for derived thermodynamic properties, especially heat capacities. In the compressed liquid region, calculated pressures will have an uncertainty of several percent. This is a consequence of the nature of the surface and is in no way the fault of the equation of state.

In each of the sections on the individual fluids, uncertainty estimates are given for that particular fluid. These estimates do not reflect the potential large uncertainties of the problem areas outlined above.

\section{Computer Routines}

The thermophysical properties produced by the computer programs listed in Appendix $C$ are all calculated using a mathematical model of the equation of state of the fluid and classical thermodynamic relationships. The reader who is interested in the thermodynamics and mathematics of the problem is referred to McCarty [7].

In general eleven or twelve significant figures are needed in the property calculations to prevent round of errors. Sample calculations for each fluid are given in Appenidx A for the purpose of checking the performance of the programs on individual computers.

Upper and lower temperature and pressure limits for each fluid have been installed in the programs according to the range of validity as claimed by the original source document. All of the functions reduce to the ideal gas in the limit of zero pressure, but because of the very large exponents in some of the terms, the iterative solution for density fails at very low pressures. Because the models are empirical and cannot be trusted to give even reasonable results outside their ranges of pressure and temperature, the user is cautioned not to change the pressure or temperature limits originally set in the programs.

\section{Acknowledgments}

The author wishes to acknowledge the support of NASA, without which this work would not have been possible, and in particular the support and encouragement of Dr. Timothy Cleghorn at the Johnson space Center. 
5. References

[1] Younglove, B. A. Interactive fortran program to calculate thermophysical properties of six fluids. Nat. Bur. Stand. (U.S.) Tech. Note 1048; 1982.

[2] Younglove, B. A. Thermophysical Properties of Fluids. I. Argon, ethylene, parahydrogen, nitrogen, nitrogen trifluoride, and oxygen. J. Phys. Chem. Ref. Data Vol. 11, Supplement No. 1 ; 1982 .

[3] Ely, J. F. Thermophysical properties of methane, ethane, propane, normal and isobutane. to be published.

[4] McCarty, R. D. Interactive FORTRAN IV conputer programs for the thermodynamic and transport properties of selected cryogens [Fluids Pack], Nat. Bur. Stand. (U.S.) Tech. Note $1025 ; 1980$.

[5] McCarty, R. D. Thermodynamic Properties of Helium 4 and 2 to $1500 \mathrm{~K}$ at Pressures to $10^{8}$ Pa. J. Phys. Chem. Ref. Data Vol. 2, No. 4, 923-1042; 1973

[6] Jacobsen, R. T.; Stewart, R. B.; McCarty, R. D.; Hanley, H. J. M. Thermophysical properties of nitrogen from the fusion line to $3500 \mathrm{R}$ [1944 K] for pressures to 150,000 psia $\left[10342 \times 10^{5} \mathrm{~N} / \mathrm{m}^{2}\right]$. Nat. Bur. Stand. (U.S.) Tech. Note 648; 1973 Dec.

[7] McCarty, R. D. Determination of thermodynamic properties from the experimental P-V-T relationships. Vol. II, Experimental thermodynamics of non-reacting fluids. LeNeindre, B.; Vodar, B., eds. Butterworth and Co., Ltd., London, England; 1975.

[8] Roder, H. M.; McCarty, R. D. A modified Benedict-Webb-Rubin equation of state for parahydrogen-II. Nat. Bur. Stand. (U.S.) NBSIR 75-814; 1975.

[9] Stewart, J. W. Dielectric polarizability of fluid parahydrogen. J. Chem. Phys. 40: 3297-3306; 1964.

[10] Weber, L. A. A modified Benedict-Webb-Rubin equation of state for gaseous and 1 iquid oxygen. Nat. Bur. Stand. (U.S.) NBSIR 78-882; 1978.

[11] Ely, J. F.; Straty, G. C. Dielectric constants and molar polarizabilities of saturated and compressed fluid nitrogen. J. Chem. Phys. 61: 1480-1485; 1974.

[12] Younglove, B. A. Dielectric constant of compressed gaseous and liquid oxygen. J. Res. Nat. Bur. Stand. (U.S.) 76A: 37-40; 1972.

[13] Stewart, R. B.; Jacobsen, R. T.; Becker, J. H. A thermodynamic property formulation for argon using a 32 term pressure explicit equation of state. Center for Applied Thermodynamic Studies Report No. 81-3; University of Idaho, Moscow, ID; 1983 July.

[14] Goodwin, R. D.; Weber, L. A. Thermophysical properties of nitrogen trifluoride from 66 to $500 \mathrm{~K}$ at pressures to 500 bar. Nat. Bur. Stand. (U.S.) NBSIR 80-1632; 1980 Jul.

[15] McCarty, R. D.; Jacobsen, R. T. An equation of state for fluid ethylene. Nat. Bur. Stand. (U.S.) Tech. Note 1045; 1981. 
Appendix A

Description of Individual Fluids 
The source of the hydrogen equation of state is NBSIR $75-814$ by Roder and McCarty [8] and Stewart $[9]$.

Estimated Uncertainties (\%)

$$
\text { Property }
$$

Pressure

Density

Temperature

Enthapy \& Internal Energy

Entropy

$\mathrm{C}_{\mathrm{p}}$ and $\mathrm{C}_{\mathrm{V}}$

Speed of Sound

\section{Fixed Points}

Critical point

Triple Point Liquid

Triple Point Vapor

Liquid Below $\mathrm{T}_{\mathrm{C}}$
5.0
0.1
0.1
1.0
1.0
3.0
2.0

Pressure, MPa

1.2838

0.00743

0.00743
Gas Below $\mathrm{T}_{\mathrm{C}}$

0.25

0.25

0.25

3.0

1.0

2.0

1.0

Density, $M / L$

15.556

0.06323

38.2143
Fluid Above $\mathrm{T}_{\mathrm{C}}$

0.20

0.20

0.20

5.10

1.0

3.0

1.0

Temperature, $\mathrm{K}$ 32.933 13.80

Temperature Range $\quad 13.8$ to $400 \mathrm{~K}$

Pressure Range $\quad 0$ to $120 \mathrm{MPa}$

Sample Calculations

THE TEMPERATURE RANGE FOR HYDROGEN IS 13.8 TO $400 \mathrm{~K}$

$(-434.8$ TO 260 F) WITH PRESSURES TO 120 MPA (17404 PSIA)

FOR ENGINEERING UNITS ENTER " 0 ", FOR METRIC EITER " 1 "

1 FOR SATURATION PROPERTIES ENTER "O", FOR FLUID ENTER "1"

0

FOR A SINGLE POINT ENTER "0", FOR A TABLE ENTER "1"

0 FOR SATURATED LIQUID ENTER "O", FOR VAPOR ENTER "1"

0 TO ENTER WITH TEMPERATURE ENTER "0", FOR PRESSURE " 1 " 1 ENTER A PRESSURE(MPA) O RESTARTS THE PROGRAM

.1

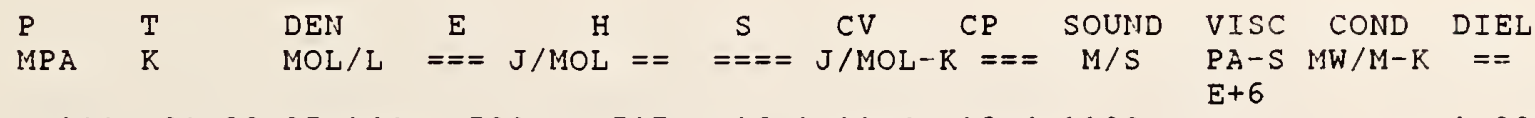
$\begin{array}{lllllllll}.100 & 20.23 & 35.144 & -520 . & -517 . & 16.1 & 11.4 & 19.4 & 1102 .\end{array}$ 1. 22999

ENTER A PRESSURE(MPA) O RESTARTS THE PROGRAM 
The source of the helium equation of state is McCarty [10].

Estimated Uncertainties $(\%)$

Property

Pressure

Density

Temperature

Enthapy \& Internal Energy

Entropy

$\mathrm{C}_{\mathrm{p}}$ and $\mathrm{C}_{\mathrm{V}}$

Speed of Sound

Thermal Conductivity

Viscosity

Fixed Points

Critical Point

Lambda Point Liquid

Lambda Point Vapor

Liquid Below $\mathrm{T}_{\mathrm{C}}$
10.0
0.5
0.5
2.0
2.0
2.0
2.0
10.0
8.0

Pressure, MPa

0.2275

0.00496

0.00496

Gas Below $\mathrm{T}_{\mathrm{C}}$
0.2
0.2
0.2
1.0
1.0
2.0
1.0
10.0
8.0

Density, $M / L$

17.399

36.5343

0.2904
Fluid Above $\mathrm{T}_{\mathrm{C}}$

0.2

0.2

0.2

1.0

1.0

0.5

0.5

10.0

10.0

Temperature, $\mathrm{K}$ 5.2014

2.172

2. 172

Temperature Range

$2-1500 \mathrm{~K}$

Pressure Range

$0-100 \mathrm{MPa}$

Sample Calculations

1

FOR SATURATION PROPERTIES ENTER "O", FOR FLUID ENTER "1"

0

FOR A SINGLE POINT ENTER "0", FOR A TABLE ENTER "१"

0

FOR SATURATED LIQUID ENTER "O", FOR VAPOR ENTER "1"

0

TO ENTER HITH TEMPERATURE ENTER "O", FOR PRESSURE "१"

1

ENTER A PRESSURE(MPA)

. 1

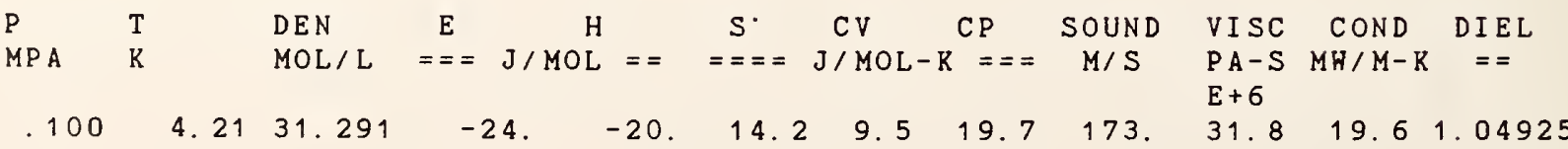


[NITROGEN]

The source of the nitrogen equation of state is Jacobsen, et al. [6] and Ely and Straty [11].

Estimated Uncertainties (\%)

Property

Pressure

Density

Temperature

Enthapy \& Internal Energy

Entropy

$\mathrm{C}_{\mathrm{p}}$ and $\mathrm{C}_{\mathrm{V}}$

Speed of Sound

Thermal Conductivity

Viscosity

\section{Fixed Points}

Critical Point

Triple Point Liquid

Triple Point Vapor

Liquid Below $\mathrm{T}_{\mathrm{C}}$
5.0
0.5
0.5
3.0
2.0
5.0
2.0
4.0
2.0

Pressure, $\mathrm{MPa}$

3.4100

0.1246

0.1246

Gas Below $\mathrm{T}_{\mathrm{C}}$
0.3
0.3
0.3
1.0
1.0
5.0
0.25
4.0
2.0

Density, $M / L$

11.21

30.977

0.2396
Fluid Above $\mathrm{T}_{\mathrm{C}}$

0.3

0.2

0.2

1.0

1.0

5.0

1.0

6.0

2. 0

Temperature, $\mathrm{K}$ 126.26

63.15

63.15

Temperature Range $\quad 63.15$ - $1900 \mathrm{~K}$

Pressure Range $\quad 0-1000 \mathrm{MPa}$

Sample Calculations

THE RANGE OF TEMPERATURE FOR NITROGEN IS 63.15 TO $1900 \mathrm{~K}$ WI TH PRESSURES TO 1000 MPA

FOR ENGINEERING UNITS ENTER " 0 ", FOR METRIC ENTER " 1 "

1 FOR SATURATION PROPERTIES ENTER " 0 ", FOR FLUID ENTER " 1 "

0 FOR A SINGLE POINT ENTER "0", FOR A TABLE ENTER "1" 0 FOR SATURATED LIQUID ENTER " 0 ", FOR VAPOR ENTER "1" 0 TO ENTER WITH TEMPERATURE ENTER " 0 ", FOR PRESSURE "1" 1 ENTER A PRESSURE (MPA)

1

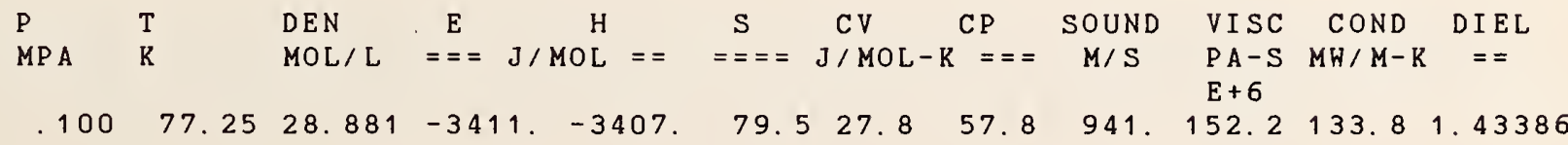




\section{[OXYGEN]}

The source of the oxygen equation of state is Weber [10] and Younglove [12].

Estimated Uncertainties (\%)

Property
Pressure
Density
Temperature
Enthapy \& Internal Energy
Entropy
$\mathrm{C}_{\mathrm{p}}$ and $\mathrm{C}_{\mathrm{V}}$
Speed of Sound
Thermal Conductivity
Viscosity

Liquid Below $\mathrm{T}_{\mathrm{C}}$
5.0
0.1
0.1
0.5
0.5
3.0
2.0
4.0
2.0

Gas Below $T_{C}$
0.25
0.25
0.20
0.25
0.25
5.0
0.50
4.0
2.0

Fluid Above $\mathrm{T}_{\mathrm{C}}$

0.15

0.15

0.10

0.50

0.50

3.0

0.50

6.0

2.0

\section{Fixed Points}

Critical Point

Pressure, MPa

Density, M/L

13.63

Temperature, $\mathrm{K}$

$$
\begin{aligned}
& 5.0422 \\
& 0.1490 \times 10^{-3} \\
& 0.1490 \times 10^{-3}
\end{aligned}
$$$$
40.820
$$$$
154.481
$$

40.820

54.359

Triple Point Vapor

$0.3275 \times 10^{-3}$

54.359

Temperature Range

54.359 to $400 \mathrm{~K}$

Pressure Range

$0-120 \mathrm{MPa}$

Sample Calculations

THE RANGE OF TEMPERATURE FOR OXYGEN IS 54.359 TO $400 \mathrm{~K}$

WITH PRESSURES TO 120 MPA

FOR ENGINEERING UNITS ENTER "O", FOR METRIC ENTER "1"

1

FOR SATURATION PROPERTIES ENTER " $0 "$, FOR FLUID ENTER "1"

0

FOR A SINGLE POINT ENTER " $0 "$, FOR A TABLE ENTER "1"

0

FOR SATURATED LIQUID ENTER "O", FOR VAPOR ENTER " 1 "

0

TO ENTER WITH TEMPERATURE ENTER "0", FOR PRESSURE " 1 "

1

ENTER A PRESSURE(MPA)

1

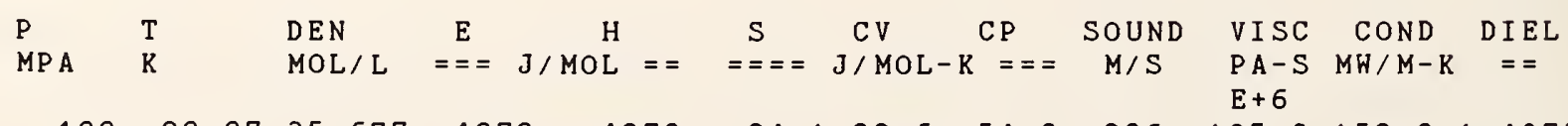

$94.129 .6 \quad 54.2 \quad 906.195 .3152 .0 \quad 1.48734$ 
The source of the argon equation of state is Stewart, et al. [13].

Estimated Uncertainties (\%)

\begin{tabular}{lccc}
\multicolumn{1}{c}{ Property } & Liquid Below $\mathrm{T}_{\mathrm{C}}$ & Gas Below $\mathrm{T}_{\mathrm{C}}$ & Fluid Above $\mathrm{T}_{\mathrm{C}}$ \\
Pressure & 10.0 & 0.3 & 0.3 \\
Density & 0.25 & 0.3 & 0.3 \\
Temperature & 0.25 & 0.3 & 0.3 \\
Enthapy \& Internal Energy & 2.0 & 1.0 & 1.5 \\
Entropy & 1.0 & 1.0 & 1.0 \\
$C_{\mathrm{p}}$ and C & 5.0 & 5.0 & 5.0 \\
Speed of Sound & 5.0 & 5.0 & 5.0 \\
Thermal Conductivity & 4.0 & 4.0 & 6.0 \\
Viscosity & 2.0 & 2.0 & 2.0
\end{tabular}

\section{Fixed Points}

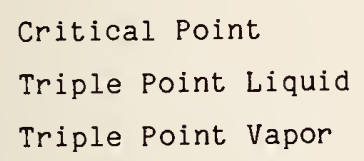

Density, M/L

$$
13.41
$$

35.40

$0.1015 \times 10^{-3}$
Temperature, $\mathrm{K}$ 150.86

83.8 83.8

Temperature Range

83.8 to $400 \mathrm{~K}$

Pressure Range

$0-100 \mathrm{MPa}$

Sample Calculations

THE TEMPERATURE RANGE FOR ARGON IS 83.8 TO $400 \mathrm{~K}$

WITH PRESSURES TO 100 MPA

FOR ENGINEERING UNITS ENTER " 0 ", FOR METRIC ENTER " 1 "

1

FOR SATURATION PROPERTIES ENTER " $0 "$, FOR FLUID ENTER "1"

0

FOR A SINGLE POINT ENTER "O", FOR A TABLE ENTER "1"

0

FOR SATURATED LIQUID ENTER "O", FOR VAPOR ENTER " 1 "

0

TO ENTER WITH TEMPERATURE ENTER "0", FOR PRESSURE "1"

1

ENTER A PRESSURE( MPA)

$\cdot 1$

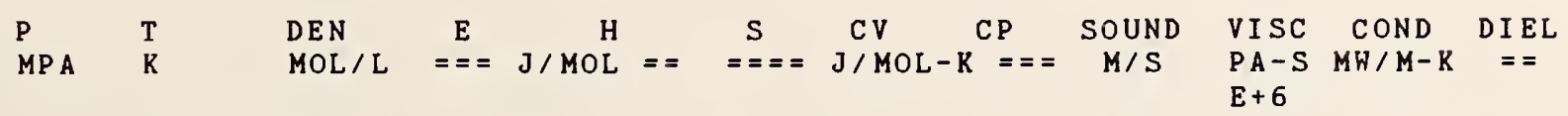

$\begin{array}{lllllllllll}.100 & 87.16 & 34.914 & -4714 . & -4711 . & 54.8 & 22.3 & 44.7 & 815 . & 261.2 & 128.5\end{array}$ 
The source of the nitrogen trifluoride equation of state is Goodwin and Weber [14].

Estimated Uncertainties $(\%)^{*}$

$\quad$ Property
Pressure
Density
Temperature
Enthapy \& Internal Energy
Entropy
$C_{p}$ and $C_{V}$
Speed of Sound

Liquid Below $T_{C}$
\[ \begin{array}{c}0.0 \\ 0.1 \\ 0.1 \\ 2.0 \\ 2.0 \\ 5.0 \\ 5.0\end{array} \]

Gas Below $\mathrm{T}_{C}$
0.2
0.2
0.1
2.0
2.0
5.0
5.0

Fluid Above $\mathrm{T}_{\mathrm{C}}$

0.1

0.1

0.1

2.0

2.0

5.0

5.0

Fixed Points

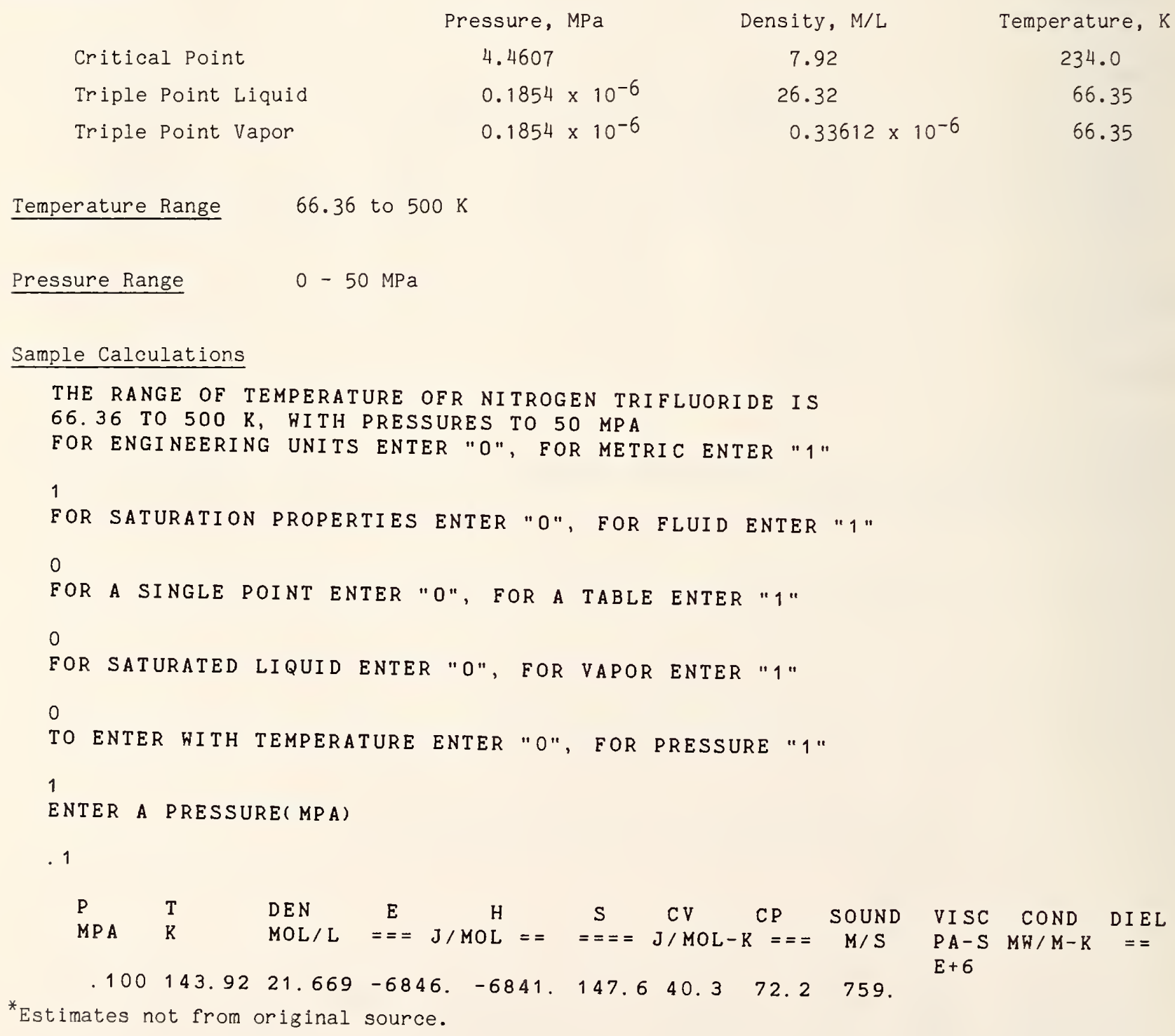


The source of the methane equation of state is Ely, et al. [3].

Estimated Uncertainties $(\%)^{*}$

Property

Pressure

Density

Temperature

Enthapy \& Internal Energy

Entropy

$\mathrm{C}_{\mathrm{p}}$ and $\mathrm{C}_{\mathrm{V}}$

Speed of Sound

Thermal Conductivity

Viscosity

\section{Fixed Points}

Critical Point

Triple Point Liquid

Triple Point Vapor

Liquid Below $\mathrm{T}_{\mathrm{C}}$
5.0
0.1
0.1
1.0
0.5
2.0
1.0
5.0
3.0

Pressure, MPa

$$
\begin{aligned}
& 4.599 \\
& 0.01174 \\
& 0.01174
\end{aligned}
$$

Gas Below $\mathrm{T}_{\mathrm{C}}$
0.25
0.25
0.25
0.5
0.5
5.0
0.3
5.0
3.0

Density, $M / L$

10.23

28. 1511

$0.01569 \times 10^{-6}$
Fluid Above $\mathrm{T}_{\mathrm{C}}$

0.25

0.25

0.25

0.50

0.50

2.00

0.3

4.0

2.0

Temperature, $\mathrm{K}$ 190.55 90.68 90.68

Temperature Range $\quad 90.68$ to $600 \mathrm{~K}$

Pressure Range

$$
0-200 \mathrm{MPa}
$$

Sample Calculations

THE RANGE OF TEMPERATURE FOR METHANE IS 90.68 TO $600 \mathrm{~K}$ HITH PRESSURES TO 200 MPA

FOR ENGINEERING UNITS ENTER " 0 ", FOR METRIC ENTER " 1 "

1

FOR SATURATION PROPERTIES ENTER " 0 ", FOR FLUID ENTER "1"

0

FOR A SINGLE POINT ENTER "O", FOR A TABLE ENTER "1"

0

FOR SATURATED LIQUID ENTER " $0 "$, FOR VAPOR ENTER " $1 "$

0

TO ENTER WITH TEMPERATURE ENTER "O", FOR PRESSURE " 1 "

ENTER A PRESSURE(MPA)

.1

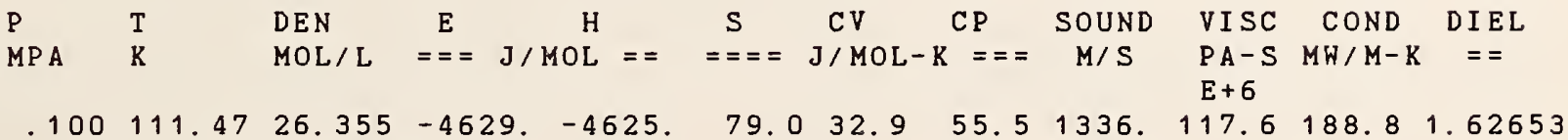

*Estimates not from original source. 
[ETHYLENE]

The source of the ethylene equation of state is McCarty and Jacobsen [15].

Estimated Uncertainties $(\%)^{*}$

$$
\text { Property }
$$

Pressure

Density

Temperature

Enthapy \& Internal Energy

Entropy

$\mathrm{C}_{\mathrm{p}}$ and $\mathrm{C}_{\mathrm{v}}$

Speed of Sound

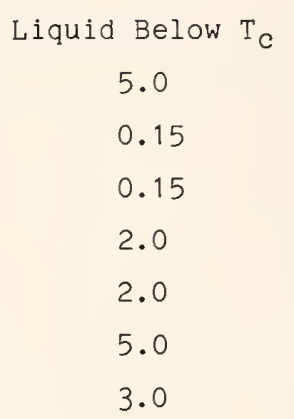

Pressure, $\mathrm{MPa}$

Critical Point

Triple Point Liquid

Triple Point Vapor

$$
\begin{aligned}
& 5.0403 \\
& 0.12 \times 10^{-3} \\
& 0.12 \times 10^{-3}
\end{aligned}
$$

Gas Below $T_{C}$
0.15
0.15
0.15
1.0
1.0
2.0
1.0

Density, M/L

7.634

23.389

$0.14 \times 10^{-3}$
Fluid Above $T_{C}$

0.15

0.15

0.15

1.0

1.0

2.0

1.0

Temperature, $\mathrm{K}$ 282.343 103.986

103.986

Temperature Range $\quad 104$ to $400 \mathrm{~K}$

Pressure Range $\quad 0-40 \mathrm{MPa}$

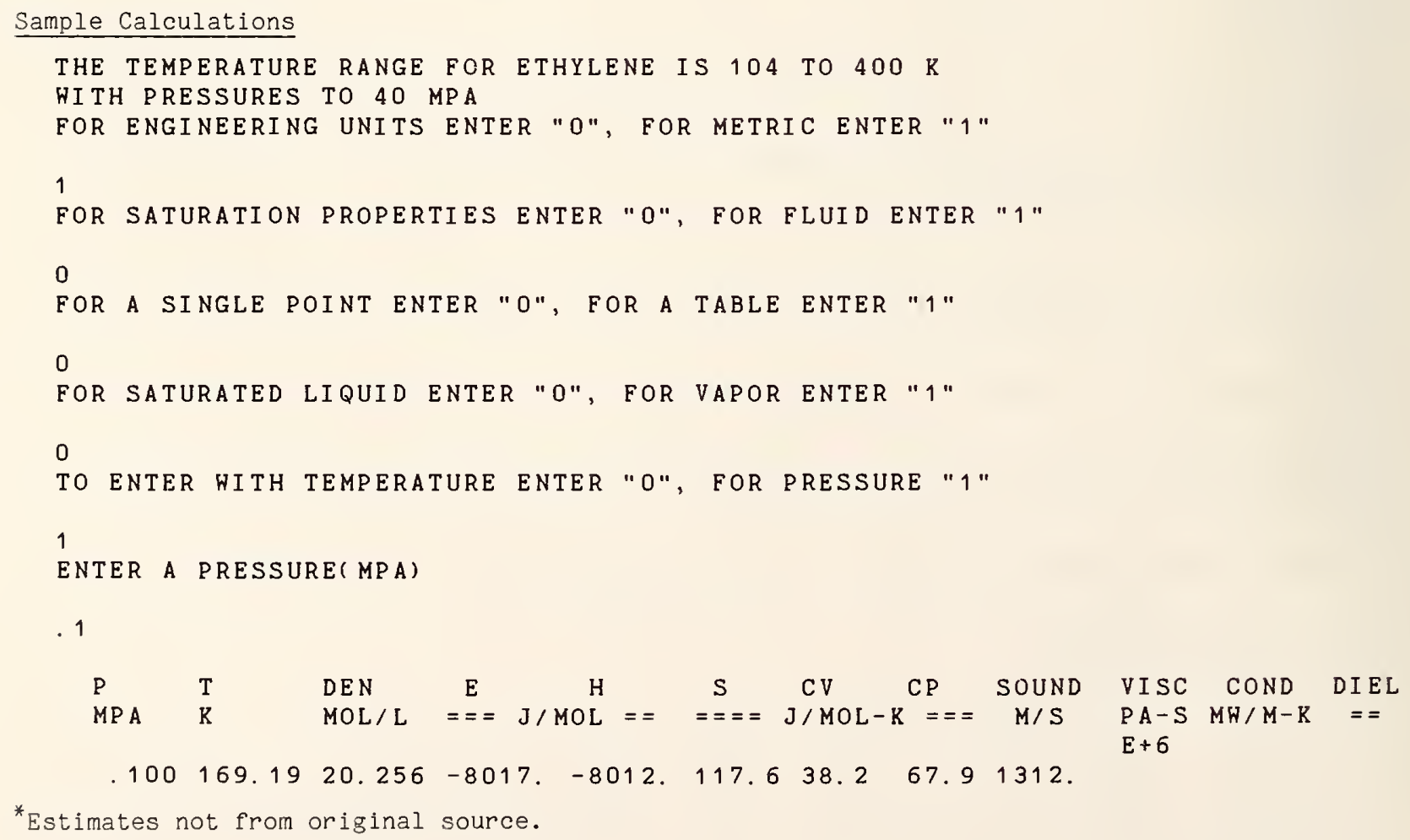


[PROPANE]

The source of the propane equation of state is Ely, et al. [3].

Estimated Uncertainties $(\%)^{*}$

Property

Pressure

Density

Temperature

Enthapy \& Internal Energy

Entropy

$\mathrm{C}_{\mathrm{p}}$ and $\mathrm{C}_{\mathrm{V}}$

Speed of Sound

Thermal Conductivity

viscosity

\section{Fixed Points}

Critical Point

Triple Point Liquid

Triple Point Vapor

Liquid Below $T_{C}$
5.0
0.1
0.1
1.0
1.0
5.0
5.0
5.0
3.0

$$
\begin{aligned}
& \text { Pressure, } \mathrm{MPa} \\
& 4.247 \\
& 0.1690 \times 10^{-9} \\
& 0.1690 \times 10^{-9}
\end{aligned}
$$

Gas Below T
0.25
0.25
0.25
1.0
1.0
5.0
5.0
5.0
3.0

Density, M/L

5.00

16.630

$0.2238 \times 10^{-9}$
Fluid Above $T_{C}$

0.15

0.15

0.15

1.5

1.5

5.0

5.0

5.0

3.0

Temperature Range $\quad 85.47$ to $600 \mathrm{~K}$

Pressure Range $\quad 0-100 \mathrm{MPa}$

Sample Calculations

THE RANGE OF TEMPERATURE FOR PROPANE IS 85.47 TO $600 \mathrm{~K}$

WITH PRESSURES TO 100 MPA

FOR ENGINEERING UNITS ENTER "O", FOR METRIC ENTER "1"

1

FOR SATURATION PROPERTIES ENTER " 0 ", FOR FLUID ENTER " $1 "$

0

FOR A SINGLE POINT ENTER "0", FOR A TABLE ENTER "1"

0

FOR SATURATED LIQUID ENTER " $0 "$, FOR VAPOR ENTER " $1 "$

0

TO ENTER HITH TEMPERATURE ENTER " 0 ", FOR PRESSURE " $1 "$

1

ENTER A PRESSURE( MPA)

.1

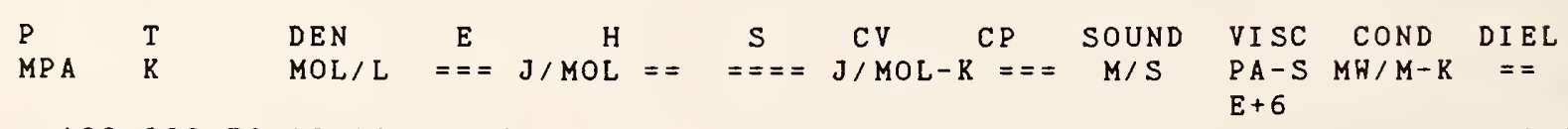

$.100230 .7813 .188-8826 . \quad-8818.171 .0 \quad 64.0 \quad 99.01160$.

1. 80350

* Estimates not from original source. 
The source of the isobutane equation of state is Ely, et al. [3].

Estimated Uncertainties $(\%)^{*}$

Property

Pressure
Density
Temperature
Enthapy \& Internal En
Entropy
$\mathrm{C}_{\mathrm{p}}$ and $\mathrm{C}_{\mathrm{v}}$
Speed of Sound
Thermal Conductivity
Viscosity

Fixed Points

Liquid Below $\mathrm{T}_{\mathrm{C}}$
$\begin{gathered}5.0 \\ 0.1 \\ 0.1 \\ 1.0 \\ 1.0 \\ 3.0 \\ 3.0 \\ 5.0 \\ 3.0\end{gathered}$

Pressure, MPa

$$
3.690
$$

$0.19481 \times 10^{-7}$

$0.19481 \times 10^{-7}$

Gas Below $\mathrm{T}_{\mathrm{C}}$
0.20
0.20
0.20
1.0
1.0
3.0
3.0
5.0
3.0

Density, $M / L$

3.86

12.755

$0.20633 \times 10^{-7}$
Fluid Above $\mathrm{T}_{\mathrm{C}}$

0.15

0.15

0.15

1.0

1.0

3.0

3.0

5.0

3.0

Triple Point Vapor

Temperature Range $\quad 113.55$ to $600 \mathrm{~K}$

Pressure Range $\quad 0-35 \mathrm{MPa}$

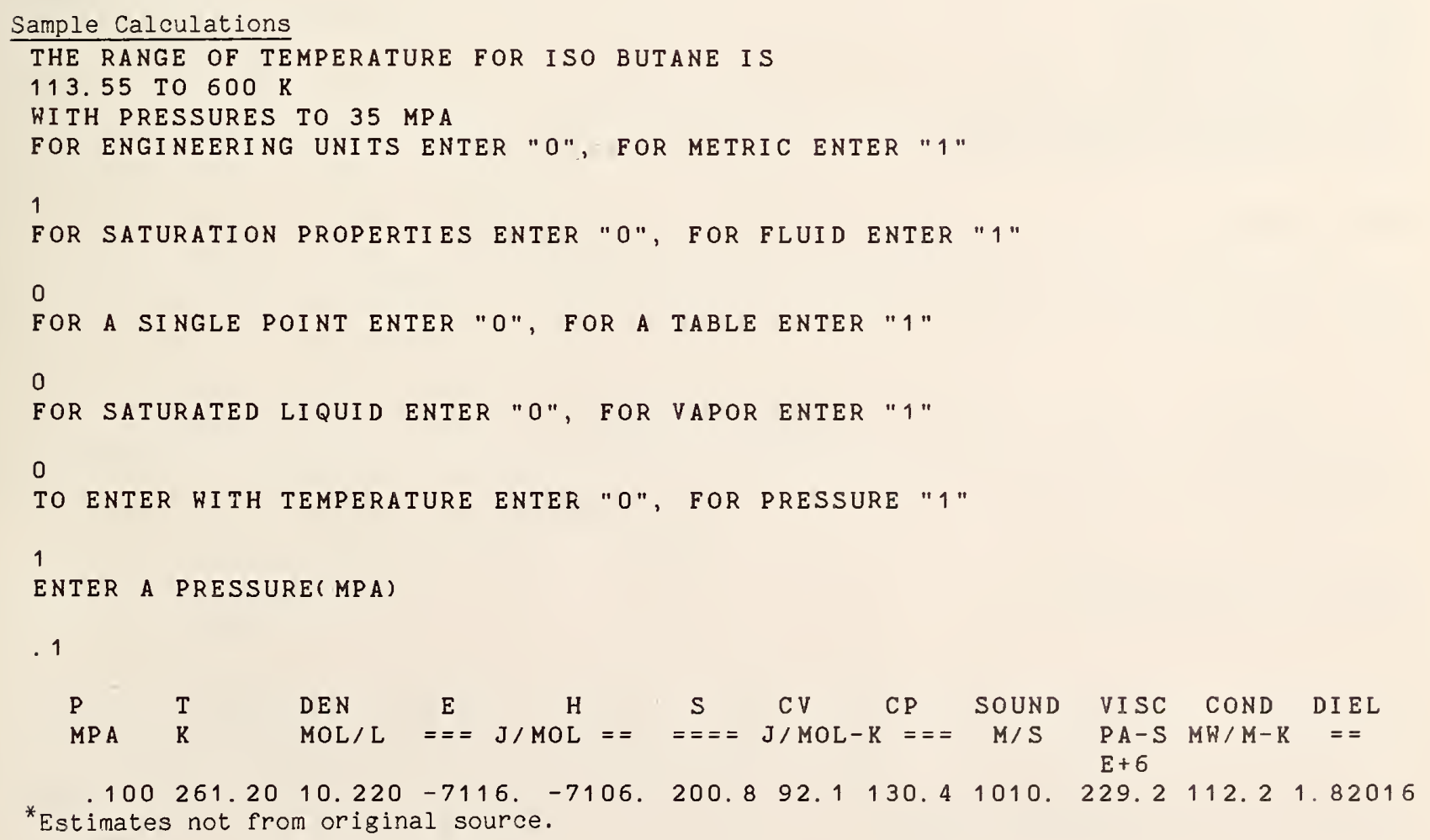


The source of the normal butane equation of state is Ely, et al. [3].

\section{Estimated Uncertainties $(\%)^{*}$}

Property

Pressure

Density

Temperature

Enthapy \& Internal Energy

Entropy

$\mathrm{C}_{\mathrm{p}}$ and $\mathrm{C}_{\mathrm{V}}$

Speed of Sound

Thermal Conductivity

viscosity

Liquid Below $\mathrm{T}_{\mathrm{C}}$
5.0
0.1
0.1
1.0
1.0
3.0
3.0
5.0
3.0

Pressure, $\mathrm{MPa}$

Critical Point

Triple Point Liquid

Triple Point Vapor
3.796
$0.6736 \times 10^{-6}$
$0.6736 \times 10^{-6}$

Gas Below $T_{C}$
0.20
0.20
0.20
1.0
1.0
3.0
3.0
5.0
3.0

Density, M/L

3.92

12.65

$0.60071 \times 10^{-6}$
Fluid Above $T_{C}$

0.15

0.15

0.15

1.0

1.0

3.0

3.0

5.0

3.0

Temperature, $\mathrm{K}$

425.16

134.86

134.86

Temperature Range $\quad 134.65$ to $500 \mathrm{~K}$

Pressure Range

$0-70 \mathrm{MPa}$

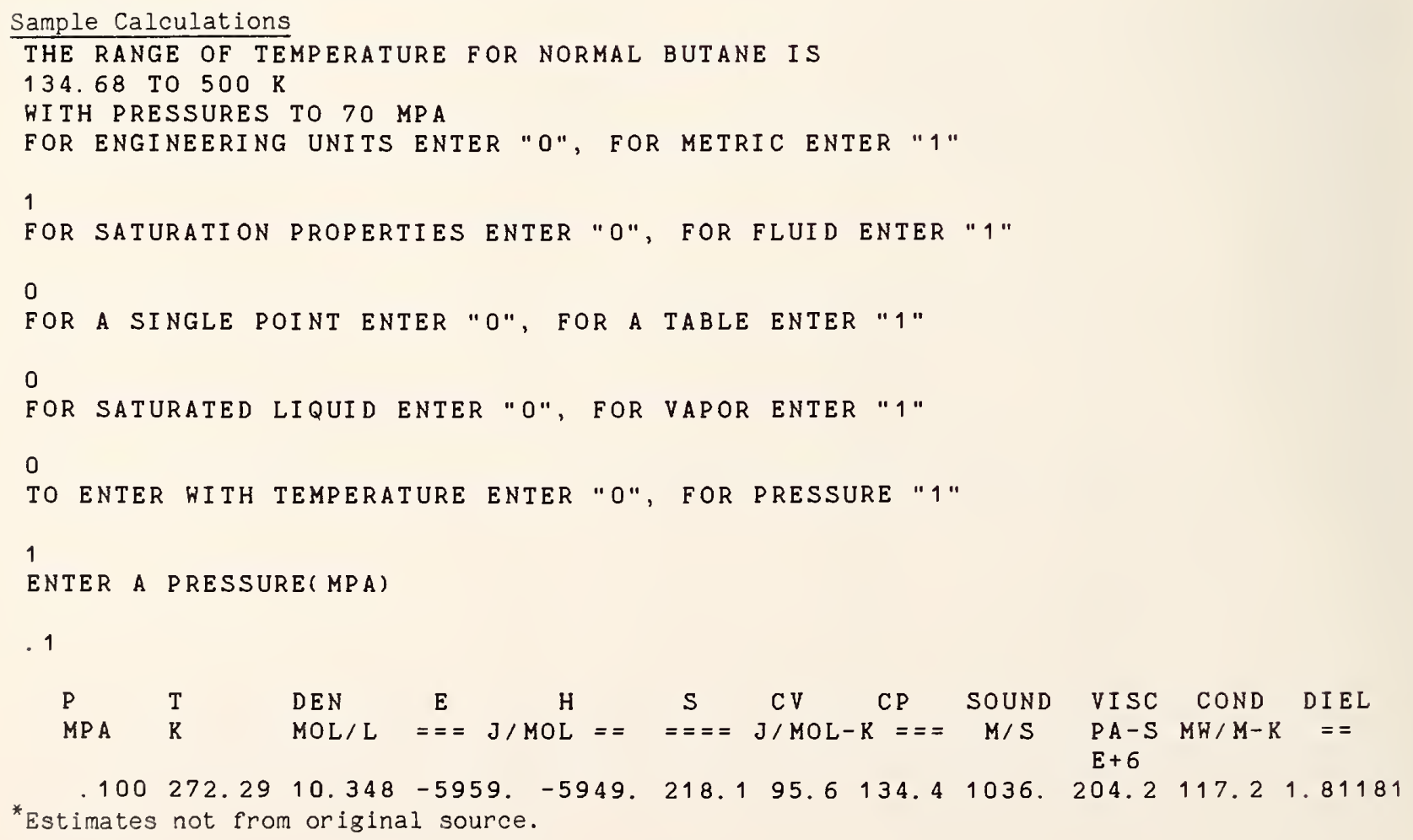


Appendix B

Sample Computer Sessions 
he 1 i um

THIS PROGRAM PROUIDES THE THERMODYNAMIC PROPERTIES OF HELIUM FROM 2 TO $1500 \mathrm{~K}$ WITH PRESSURES TO 100 MPA

WHEN THE PROGRAM ASKS FOR A PRESSURE, DENSITY AND TEMPERATURE, ENTER ANY TWO AND A ZERO FOR THE THIRD.

TO TERMINATE THE PROGRAM ENTER ZERO FOR ALL THREE.

FOR ENGINEERING UNITS ENTER "O", FOR METRIC ENTER " 1 "

1

FOR SATURATION PROPERTIES ENTER "O", FOR FLUID ENTER "1"

1

FOR A SINGLE POINT ENTER "O", FOR A TABLE ENTER "1"

1

ENTER A PRESSURE (MPA), A STARTING TEMPERATURE(K), A FINAL TEMPERATURE AND A TEMPERATURE INCREMENT AND IN THAT ORDER, TO RESTART PROGRAM ENTER "O,0,0,0"

$1,3,10,1$

\begin{tabular}{|c|c|c|c|c|c|c|c|c|c|c|c|}
\hline IPA & $\mathrm{T}$ & $\begin{array}{l}\text { DEN } \\
M O L / L\end{array}$ & $={ }^{E} \quad \mathrm{~J} / \mathrm{MOL}^{\mathrm{H}}$ & $-1=$ & $\begin{array}{l}S \\
===\end{array}$ & $\begin{array}{l}\mathrm{CV} \\
\mathrm{J} / \mathrm{MOL}-\mathrm{K}\end{array}$ & $\begin{array}{l}C P \\
===\end{array}$ & $\begin{array}{l}\text { SOUND } \\
M / S\end{array}$ & $\begin{array}{l}\text { UISC } \\
\text { PA-S } \\
F+6\end{array}$ & $\begin{array}{l}\text { COND } \\
M W / M-K\end{array}$ & $\begin{array}{l}\text { DIEL } \\
==\end{array}$ \\
\hline $\begin{array}{l}.100 \\
.100 \\
.100 \\
.100 \\
.100 \\
.100 \\
.100 \\
.100\end{array}$ & $\begin{array}{r}3.00 \\
4.00 \\
5.00 \\
6.00 \\
7.00 \\
8.00 \\
9.00 \\
10.00\end{array}$ & $\begin{array}{r}35.794 \\
32.477 \\
2.935 \\
2.252 \\
1.855 \\
1.586 \\
1.389 \\
1.238\end{array}$ & $\begin{array}{r}-39 . \\
-27 . \\
52 . \\
67 \\
81 . \\
94 . \\
107 . \\
120 .\end{array}$ & $\begin{array}{l}-36 . \\
-24 . \\
86 . \\
111 . \\
135 . \\
157 . \\
179 . \\
201 .\end{array}$ & $\begin{array}{l}9.8 \\
13.3 \\
39.1 \\
43.7 \\
47.3 \\
50.3 \\
52.9 \\
55.2\end{array}$ & $\begin{array}{r}7.6 \\
9.1 \\
12.7 \\
12.5 \\
12.5 \\
12.5 \\
12.5\end{array}$ & $\begin{array}{l}9.4 \\
16.3 \\
27.1 \\
24.0 \\
22.8 \\
22.2 \\
21.9 \\
21.7\end{array}$ & & & $\begin{array}{l}18.1 \\
19.6 \\
11.1 \\
12.4 \\
13.8 \\
15.1 \\
16.3 \\
17.5\end{array}$ & \\
\hline
\end{tabular}

ENTER A PRESSURE(MPA), A STARTING TEMPERATURE(K), A FINAL TEMPERATURE AND A TEMPERATURE INCREMENT AND IN THAT ORDER, TO RESTART PROGRAM ENTER " $0,0,0,0 "$

$0,0,0,0$

FOR MORE PROPERTIES ENTER O, TO TERMINATE ENTER 1 1

Stop - Program terminated. 
MI PROPS

FOR INFORMATION ON HOH TO USE THIS PROGRAM, ENTER "O" OTHERHISE, ENTER. " 1 "

0

ENTER A NUMBER TO SELECT A FLUID.

ANY ENTRY OTHER THAN A NUMBER HILL TERMINATE THE PROGRAM.

HHEN THE PROGRAM ASRS FOR A PRESSURE, DENSITY AND TEMPERATURE,

ENTER ANY THO AND A ZERO FOR THE THIRD. TO SELECT ANOTHER

FLUID ENTER ZERO FOR ALL THREE.

SELECT A FLUID BY ENTERING THE CORRESPONDING NUMBER

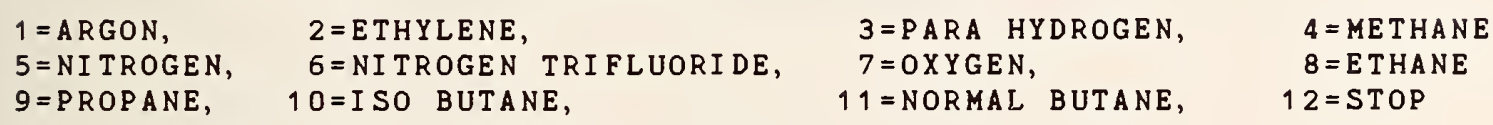

1

THE TEMPERATURE RANGE FOR ARGON IS 83.8 TO $400 \mathrm{~K}$

HITH PRESSURES TO 100 MPA

FOR ENGINEERING UNITS ENTER " 0 ", FOR METRIC ENTER " 1 "

1

FOR SATURATION PROPERTIES ENTER "O", FOR FLUID ENTER " 1 "

1

FOR A SINGLE POINT ENTER "O", FOR A TABLE ENTER "1"

1

ENTER A PRESSURE(MPA), A STARTING TEMPERATURE(K), A FINAL TEMPERATURE AND A

TEMPERATURE INCREMENT AND IN THAT ORDER, TO RESTART PROGRAM ENTER "0, $0,0,0 "$

$1,200,300,20$

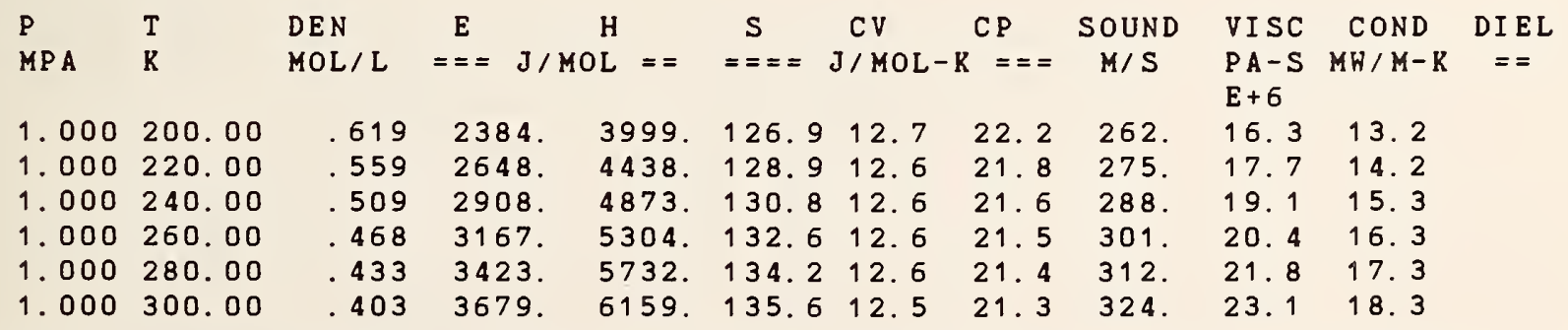

ENTER a PRESSURE( MPa), A STARTINg temperature( $R$ ), A Final temperature and a TEMPERATURE I NCREMENT AND IN THAT ORDER, TO RESTART PROGRAM ENTER " $0,0,0,0 "$ 
Appendix C

Program Listings 


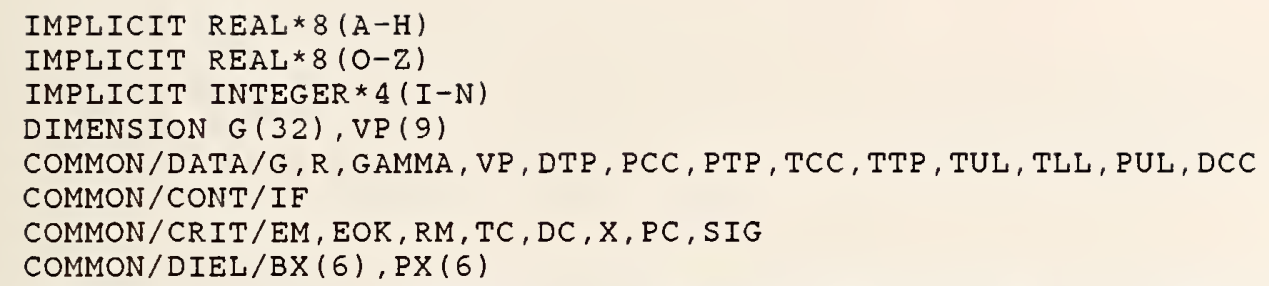

1201 FORMAT $/ / /$, THIS PROGRAM PROVIDES THE THERMODYNAMIC PROPERTIES OF 111 FLUIDS', $/$, PARA HYDROGEN, NITROGEN, OXYGEN, ARGON $2, \quad$ NITROGEN TRIFLOURIDE, METHANE, ETHANE, ETHYLENE' /

1000 FORMAT(//, FOR INFORMATION ON HOW TO USE THIS PROGRAM, ENTER "O" ' A ' OTHERWISE, ENTER " $1 " 1 / 1)$

WRITE $(*, 1000)$

$I P=3$

$\operatorname{READ}(*, 1010) I$

IF (I.EQ. O) CALL INFO

1010 FORMAT (I1)

110 WRITE $(*, 1020)$

WRITE $(*, 1030)$

1020 FORMAT(' SELECT A FLUID BY ENTERING THE CORRESPONDING NUMBER',/)

1030 FORMAT(' 1=PARA HYDROGEN, 2=NITROGEN, $3=O X Y G E N$,

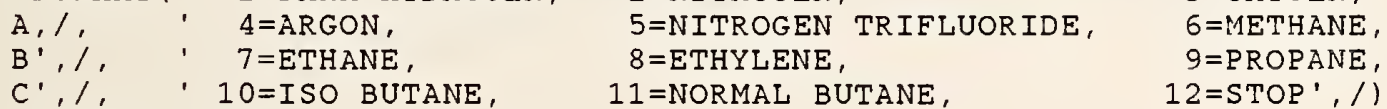

$$
\operatorname{READ}(*, *) I F
$$

$120 \operatorname{WRITE}(*, 1040)$

1040 FORMAT(" FOR ENGINEERING UNITS ENTER "0", FOR METRIC ENTER "1"',/) $\operatorname{READ}(*, 1010)$ IU WRITE $(*, 1050)$

1050 FORMAT(' FOR SATURATION PROPERTIES ENTER "O", FOR FLUID ENTER ' $A$ A " $1 ", /)$

$\operatorname{READ}(*, 1010)$ IC

WRITE $(*, 1060)$

1060 FORMAT(" FOR A SINGLE POINT ENTER "0", FOR A TABLE ENTER "1",,/) $\operatorname{READ}(*, 1010) \mathrm{IV}$

$160 \mathrm{IF}(\mathrm{IC} . \mathrm{EQ} .0)$ GO TO 240

IF (IV.EQ.1)GO TO 330

170 IF (IU.EQ.0) GO TO 180

WRITE $(*, 1080)$

$\operatorname{READ}(*, *) \mathrm{P}, \mathrm{D}, \mathrm{T}$

GO TO 190

180 WRITE $(*, 1070)$

1070 FORMAT(' ENTER PRESSURE(PSIA), DENSITY(LB/CU FT), AND TEMPERATURE' 


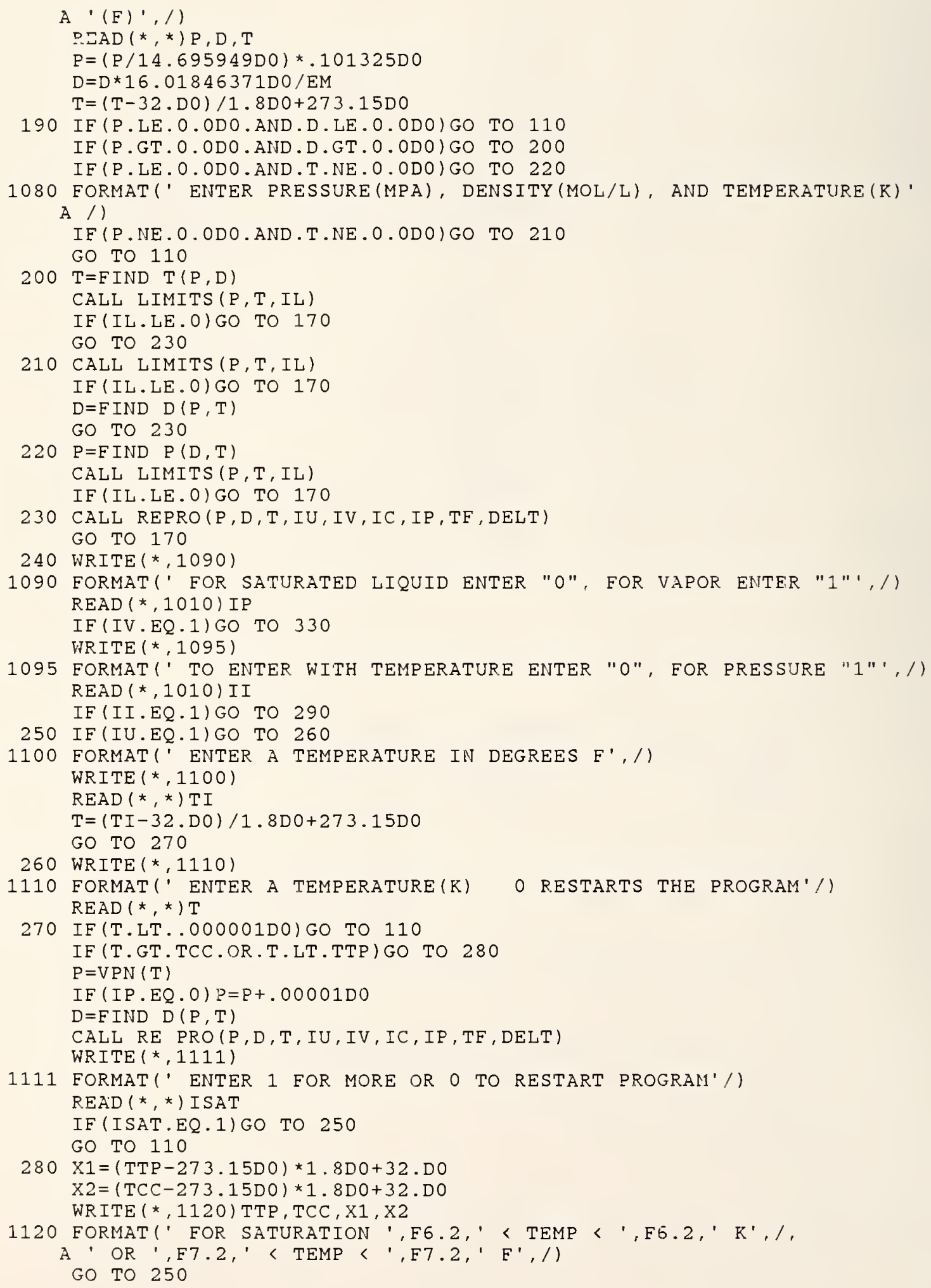


290 IF (IU.EQ.1)GO TO 300

WRITE $(*, 1130)$

1130 FORMAT(' ENTER A PRESSURE IN LB/SQ IN O RESTARTS THE PROGRAM'/)

$\operatorname{READ}(*, *) P I$

IF (PI.LE. O.ODO)GO TO 110

$\mathrm{P}=(\mathrm{PI} / 14.695949 \mathrm{DO}) * .101325 \mathrm{DO}$

GO TO 310

300 WRITE $(*, 1140)$

1140 FORMAT(' ENTER A PRESSURE(MPA) O RESTARTS THE PROGRAM'/)

$\operatorname{READ}(*, *) P I$

IF (PI.LE. O.ODO) GO TO 110

$\mathrm{P}=\mathrm{PI}$

310 IF (P.GT.PCC.OR.P.LT.PTP) GO TO 320

4000 FORMAT (D20.5)

$\mathrm{T}=\mathrm{F} I N D \quad \mathrm{TV}(\mathrm{P})$

$\mathrm{P}=\mathrm{VPN}(\mathrm{T})$

IF (IP.EQ.0) $P=P+.00001 D 0$

$D=F I N D \quad D(P, T)$

CALL RE PRO ( $P, D, T, I U, I V, I C, I P, T E, D E L T)$

GO TO 290

$320 \mathrm{PTPF}=\mathrm{PTP} * 14.695949 \mathrm{DO} / .101325 \mathrm{D} 0$

$\mathrm{PCCF}=\mathrm{PCC} \star 14.695949 \mathrm{DO} / .101325 \mathrm{DO}$

WRITE $(*, 1150)$ PTP, PCC, PTPF, PCCF

1150 FORMAT (' YOUR INPUT PRESSURE IS OUTSIDE THE RANGE OF SATURATION'

A ' PRESSURES' $/$ FOR THIS FLUID THE RANGE IS ', F6.5,' TO ', F6.3,

B ' MPA'/' OR ',F6.2,' TO ',F6.1,' PSIA'/' TRY AGÁN',/)

GO TO 290

330 IF (IC.EQ.1) GO TO 370

IF (IU.EQ.1) GO TO 340

WRITE $(*, 1160)$

1160 FORMAT (' ENTER A STARTING TEMPERATURE, A FINAL TEMPERATURE'

A /" AND A TEMPERATURE INCREMENT, IN DEGREES $F$ AND IN THAT ORDER"/)

$\operatorname{READ}(*, *) T S, T F, D E L T$

IF (DELT.LE. O.ODO) GO TO 110

$\mathrm{TS}=(\mathrm{TS}-32 . \mathrm{DO}) / 1.8 \mathrm{D} 0+273.15 \mathrm{D} 0$

$\mathrm{TF}=(\mathrm{TF}-32 . \mathrm{D} 0) / 1.8 \mathrm{D} 0+273.15 \mathrm{DO}$

DELT $=D E L T / 1.8 D 0$

IF (TS.LT.TTP.OR.TS.GT.TCC) GO TO 360

IF (TF.LT.TTP.OR.TF.GT.TCC) GO TO 360

GO TO 350

340 WRITE $(*, 1170)$

1170 FORMAT(' ENTER A STARTING TEMPERATURE, A FINAL TEMPERATURE'

1/' AND A TEMPERATURE INCREMENT IN KELVINS AND IN THAT ORDER',/)

$\operatorname{READ}(*, \star) T S, T F, D E L T$

IF (DELT.LE.O.ODO)GO TO 110

IF (TS.LT.TTP.OR.TS.GT.TCC) GO TO 360

IF (TF.LT.TTP.OR.TF.GT.TCC) GO TO 360

$350 \mathrm{~T}=\mathrm{TS}$

$P=V P N(T)$

IF (IP.EQ.0.0DO) $P=P+.00001 D 0$

$\mathrm{D}=\mathrm{F}$ IND $\mathrm{D}(\mathrm{P}, \mathrm{T})$

CALL RE PRO(P,D, T, IU, IV, IC, IP, TF, DELT)

GO TO 330

$360 \times 1=(T T P-273.15 D 0) * 1.8 D 0+32 . D 0$

$\mathrm{X} 2=(\mathrm{TCC}-273.15 \mathrm{D} 0) \star 1.8 \mathrm{D} 0+32 . \mathrm{D} 0$

WRITE $(*, 1180)$ TTP , TCC $, X 1, X 2$

1180 FORMAT(' FOR SATURATION, ',F6.2,'< 'TEMP<',F6.2,' $\mathrm{K}^{\prime}, 1$,

A , 13X, OR, ',F7.1,' ( TEMP<', F7.1,' F. TRY AGAIN.', /)

GO TO 330 


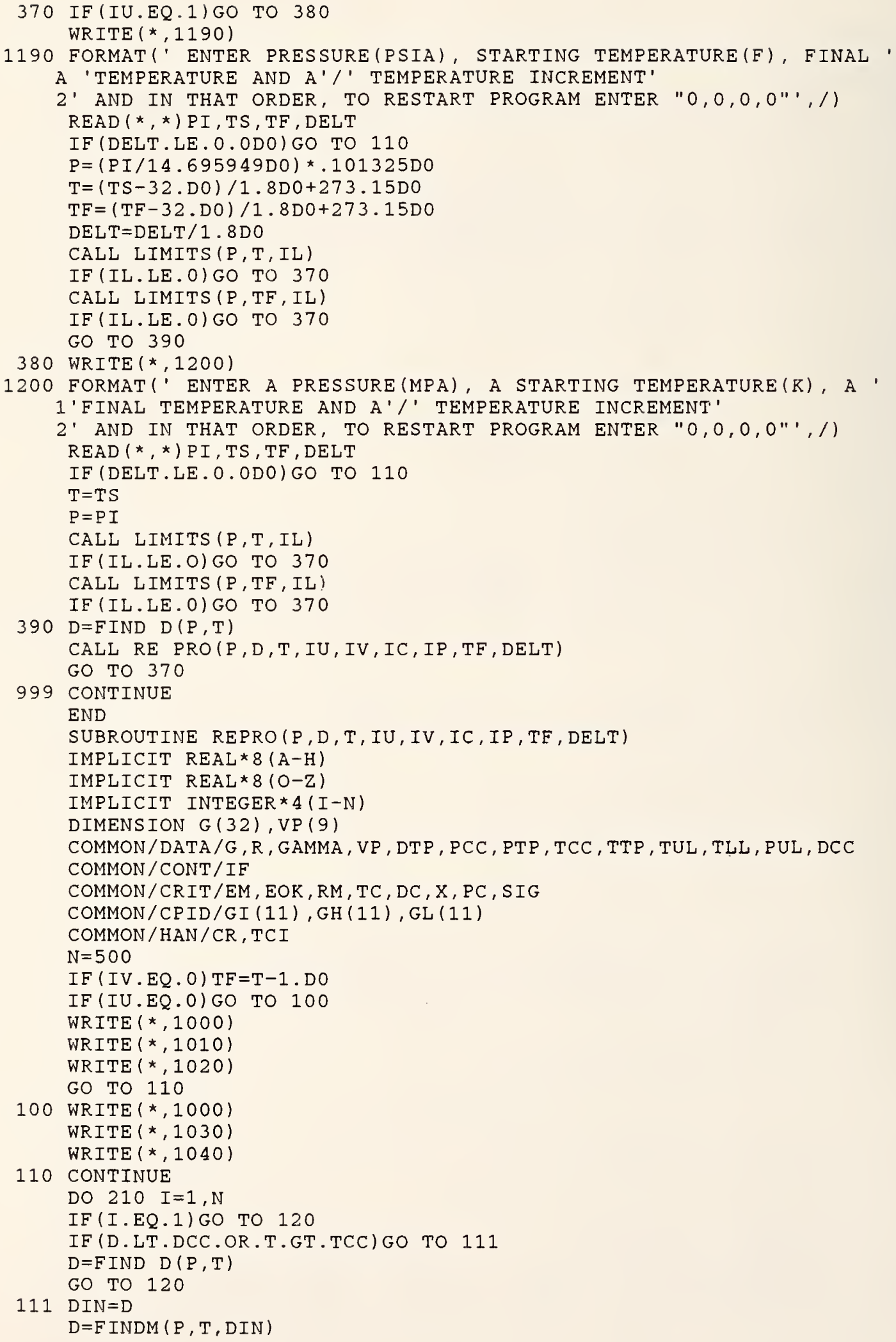


$120 \mathrm{H}=\mathrm{ENTHAL}(\mathrm{P}, \mathrm{D}, \mathrm{T})$

$\mathrm{E}=\mathrm{H}-1000 . \mathrm{DO} * \mathrm{P} / \mathrm{D}$

$S=E N T R O P(D, T)$

$W=\operatorname{SOUND}(D, T)$

$C P P=C P(D, T)$

$C V V=C V(D, T)$

IF (IU.EQ.0) GO TO 160

IF (IF.EQ.6.OR.IF.EQ.2.OR.IF.EQ.3) GO TO 150

IF (IF.GT.9)GO TO 150

IF (IF.EQ.4) GO TO 140

IF (IF.EQ.1.OR.IF.EQ.7.OR.IF.EQ.9) GO TO 130

WRITE $(\star, 2000) \quad P, T, D, E, H, S, C V V, C P P, W$

GO TO 200

130 EPS=FDIEL $(P, D, T)$

WRITE $(*, 2010) \quad P, T, D, E, H, S, C V V, C P P, W, E P S$

GO TO 200

$140 \mathrm{TH}=\mathrm{THERM}(\mathrm{D}, \mathrm{T}) * 1000 . \mathrm{D} 0$

$\mathrm{V}=\mathrm{VISC}(\mathrm{D}, \mathrm{T})$

WRITE (*, 2020) P, T, D, E , H, S, CVV , CPP , W , V , TH

GO TO 200

$150 \mathrm{~V}=\mathrm{VISC}(\mathrm{D}, \mathrm{T})$

$\mathrm{TH}=\mathrm{THERM}(\mathrm{D}, \mathrm{T}) \star 1000 . \mathrm{DO}$

EPS $=F D I E L(P, D, T)$

WRITE $(*, 2030) \quad P, T, D, E, H, S, C V V, C P P, W, V, T H, E P S$

GO TO 200

$160 \mathrm{H}=\mathrm{H} /(2.324445 \mathrm{DO} * \mathrm{EM})$

$E=E /(2.324445 D O * E M)$

$S=S /(4.184001 D 0 * E M)$

$C P P=C P P /(4.184001 D 0 * E M)$

$\mathrm{CVV}=\mathrm{CVV} /(4.184001 \mathrm{DO} * \mathrm{EM})$

$\mathrm{W}=\mathrm{W} * 3.280840 \mathrm{DO}$

$\mathrm{PO}=(\mathrm{P} / .101325 \mathrm{DO}) * 14.695949 \mathrm{DO}$

$\mathrm{DO}=\mathrm{D} * \mathrm{EM} / 16.01846371 \mathrm{D} 0$

$\mathrm{TO}=\mathrm{T} \star 1.8 \mathrm{D} 0-459.67 \mathrm{DO}$

IF (IF.EQ.6.OR.IF.EQ.2.OR.IF.EQ.3) GO TO 190

IF (IF.EQ.4) GO TO 180

IF (IF.EQ.1) GO TO 170

WRITE $(\star, 3000)$ PO, TO,DO,E,H, S, CVV, CPP,W

GO TO 200

170 EPS $=F D I E L(P, D, T)$

WRITE $(*, 3010)$ PO, TO,DO, E, H, S, CVV , CPP,W, EPS

GO TO 200

$180 \mathrm{~V}=\mathrm{VISC}(\mathrm{D}, \mathrm{T}) * .067196897 \mathrm{DO}$

$\mathrm{TH}=\mathrm{THERM}(\mathrm{D}, \mathrm{T}) * .578176 \mathrm{DO}$

WRITE (*, 3020) PO, TO,DO, E, H, S , CVV, CPP, W, V , TH

GO TO 200

$190 \mathrm{~V}=\mathrm{VISC}(\mathrm{D}, \mathrm{T}) * .067196897 \mathrm{DO}$

$\mathrm{TH}=\mathrm{THERM}(\mathrm{D}, \mathrm{T}) \star .578176 \mathrm{DO}$

$E P S=F D I E L(P, D, T)$

WRITE $(*, 3030)$ PO, TO,DO, E, H, S, CVV , CPP,W, V , TH, EPS

$200 \mathrm{~T}=\mathrm{T}+\mathrm{DELT}$

$I F(T . G T . T F+.01 D O) G O$ TO 220

$I F(I C . E Q \cdot 0) P=V P N(T)$

$I F(I P . E Q .0) P=P+.00001 D 0$

210 CONTINUE

220 CONTINUE

WRITE (*, 1000)

1000 FORMAT(' ')

RETURN 


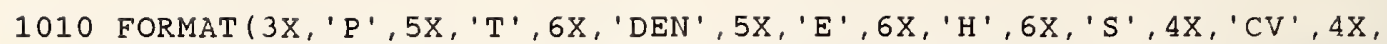

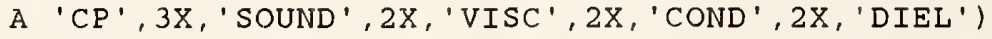

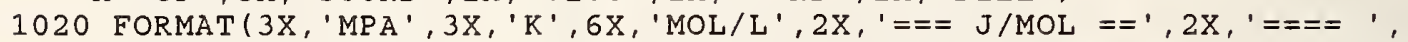

$\left.\mathrm{A}^{\prime} \mathrm{J} / \mathrm{MOL}-\mathrm{K}==={ }^{\prime}, 2 \mathrm{X},{ }^{\prime} \mathrm{M} / \mathrm{S}^{\prime}, 3 \mathrm{X},{ }^{\prime} \mathrm{PA}-\mathrm{S}^{\prime}, 1 \mathrm{X},{ }^{\prime} \mathrm{MW} / \mathrm{M}-\mathrm{K}^{\prime}, 2 \mathrm{X},{ }^{\prime}==\mathrm{\prime}^{\prime}, /, 61 \mathrm{X},{ }^{\prime} \mathrm{E}+6^{\prime}\right)$

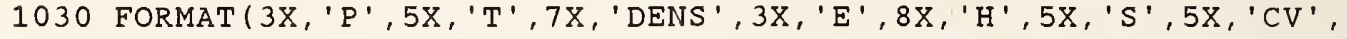

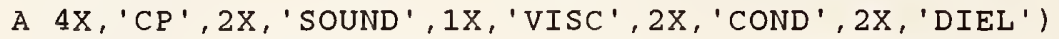

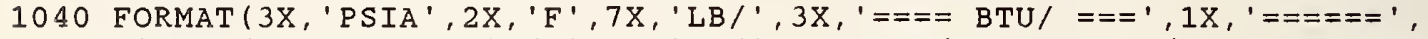

$\mathrm{A}{ }^{\prime} \mathrm{BTU} /===={ }^{\prime}, 2 \mathrm{X},{ }^{\prime} \mathrm{F} / \mathrm{S}^{\prime}, 3 \mathrm{X},{ }^{\prime} \mathrm{LB} /{ }^{\prime}, 2 \mathrm{X},{ }^{\prime} \mathrm{BTU} /{ }^{\prime}, 2 \mathrm{X},{ }^{\prime}===^{\prime}, /, 17 \mathrm{X},{ }^{\prime} \mathrm{CU} \mathrm{FT}^{\prime}$,

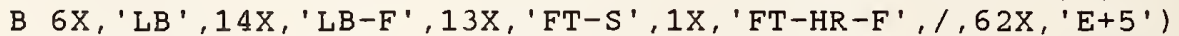

2000 FORMAT (F 8.3, F7.2, F7 .3, 2F7.0, F6.1, F5.1, F6.1, F6.0)

2010 FORMAT (F8.3,F7.2,F7.3,2F7.0,F6.1,F5.1,F6.1,F6.0,13X,F 8.5)

2020 FORMAT (F8.3,F7.2,F7.3,2F7.0,F6.1,F5.1,F6.1,F6.0,F6.1,F6.1)

2030 FORMAT (F8.3,F7.2,F7.3,2F7.0,F6.1,F5.1,F6.1,F6.0,F6.1, F6.1,F8.5)

3000 FORMAT (F8.1,F7.2, F7.3,2F7.1,3F6.3,F6.0)

3010 FORMAT (F 8.1,F7.2, F7 . 3, 2F 7.1,3F6.3, F6.0,11X, F 8.5)

3020 FORMAT (F8.1,F7.2,F7.3,2F7.1,3F6.3,F6.0,F5.2,F6.4)

3030 FORMAT (F8.1,F7.2,F7.3,2F7.1,3F6.3,F6.0,F5.2,F6.4,F8.5)

END

SUBROUTINE INFO

$\operatorname{WRITE}(*, 100)$

100 FORMAT (/' ENTER A NUMBER TO SELECT A FLUID.' $/$

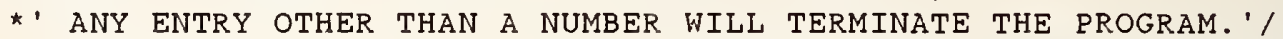

* WHEN THE PROGRAM ASKS FOR A PRESSURE, DENSITY AND TEMPERATURE, $1 /$

* ' ENTER ANY TWO AND A ZERO FOR THE THIRD. TO SELECT ANOTHER' $/$

*' FLUid ENTER ZERO FOR ALL THREE.')

RETURN

END

SUBROUTINE FDATA (IF)

IMPLICIT REAL*8 $(A-H)$

IMPLICIT REAL * $8(\mathrm{O}-\mathrm{Z})$

IMPLICIT INTEGER * $4(I-N)$

DIMENSION $\mathrm{G}(32), \mathrm{VP}(9), \mathrm{GV}(9), \mathrm{GT}(9), \mathrm{FV}(4), \mathrm{FT}(4), \mathrm{EV}(8), \mathrm{ET}(8), A(20)$

COMMON/DATA/G, R, GAMMA, VP , DTP , PCC , PTP , TCC , TTP , TUL , TLL , PUL , DCC

COMMON/SEN/BETA, XO,DELTA,E1,E2, AGAM

COMMON/CRIT/EM, EOK, RM, TC, DC , X, PC, SIG

COMMON/SATC/A, DTPV, EG

COMMON/CPID/GI (11), GH (11), GL (11)

COMMON/DATA1/GV, GT, FV, FT, EV, ET

COMMON/ISP / N, NW, NWW

COMMON/FIXPT/TO, SO, HO

COMMON/DIEL/BX (6), PX (6)

COMMON/B/GMW, SEOK, S S IG

COMMON/A/SE1, G1, B1, DE, BK, D1, XZ, ZZ, X1, X2, X3, X4

GO TO $(3,5,7,1,6,4,8,2,9,10,11,12)$, IF

1 WRITE $(*, 200)$

200 FORMAT(' THE TEMPERATURE RANGE FOR ARGON IS 83.8 TO $400 \mathrm{~K}$ '

$1 /^{\prime}(-308.8$ TO $260 \mathrm{~F})$ WITH PRESSURES TO $\left.100 \mathrm{MPA}(14504 \text { PSIA })^{\prime}\right)$

$\operatorname{OPEN}(5, F I L E=$ ' ARGON. COF')

$\mathrm{N}=0$

$\mathrm{NW}=0$

$\mathrm{NWW}=0$

GO TO 50

2 WRITE $(*, 201)$

201 FORMAT (' THE TEMPERATURE RANGE FOR ETHYLENE IS 104 TO $400 \mathrm{~K}$ '

$1{ }^{\prime}(-272.4$ TO $260 \mathrm{~F})$ WITH PRESSURES TO 40 MPA $\left.(5801 \text { PSIA })^{\prime \prime}\right)$ $\operatorname{OPEN}\left(5, \mathrm{FILE}={ }^{\prime} \mathrm{C} 2 \mathrm{H} 4 . \mathrm{COF}^{\prime}\right)$

$\mathrm{N}=0$

$\mathrm{NW}=0$

$\mathrm{NWW}=0$ 
$\mathrm{NWW}=0$

GO TO 50

3 HRITE $(\star, 202)$

202 FORMAT( ' THE TEMPERATURE RANGE FOR HYDROGEN IS 13.8 TO $400 \mathrm{~K}$ '

$1 /^{\prime}$ WITH PRESSURES TO $120 \mathrm{MPA}^{\prime}$ )

OPEN ( 5, FILE =' PH2. COF')

$N=1$

$\mathrm{NH}=0$

$\mathrm{NWW}=0$

GO TO 50

4 HRITE( $\star 2,203)$

203 FORMAT(' THE RANGE OF TEMPERATURE FOR METHANE IS 90.68 TO $600 \mathrm{~K}$ '

$1 /$ HITH PRESSURES TO 200 MPA')

OPEN( 5, FILE=' METH. COF')

$\mathrm{N}=0$

$\mathrm{NW}=1$

$\mathrm{NHW}=0$

GO TO 50

5 HRITE $(\star, 204)$

204 FORMAT(' THE RANGE OF TEMPERATURE FOR NITROGEN IS 63.15 TO $1900 \mathrm{~K}$ '

$1 /$. HITH PRESSURES TO 1000 MPA')

OPEN ( 5, FILE=' N2. COF')

$\mathrm{N}=0$

$\mathrm{NW}=0$

NWW $=0$

GO TO 50

6 WRITE $(\star, 205)$

205 FORMAT( ' THE RANGE OF TEMPERATURE OFR NITROGEN TRIFLUORIDE IS'

$1, l^{\prime} 66.36$ TO $500 \mathrm{~K}$, WITH PRESSURES TO $50 \mathrm{MPA}^{\prime}$ )

OPEN( 5, FILE =' NF 3. COF')

$\mathrm{N}=0$

$\mathrm{NH}=0$

$\mathrm{NWH}=0$

GO TO 50

7 HRITE $(\star, 206)$

206 FORMAT(' THE RANGE OF TEMPERATURE FOR OXYGEN IS 54.359 TO $400 \mathrm{~K}$ '

$1 /$ WITH PRESSURES TO $120 \mathrm{MPA}^{\prime}$ )

OPEN( $5, F I L E={ }^{\prime} 02$. COF' )

$\mathrm{N}=0$

$\mathrm{NH}=0$

$\mathrm{NHW}=0$

GO TO 50

8 HRITE $(\star, 207)$

207 FORMAT( ${ }^{\circ}$ THE RANGE OF TEMPERATURE FOR ETHANE IS 90.35 TO $600 \mathrm{~K}$ '

$1 /$ HITH PRESSURES TO 70 MPA')

OPEN( 5, FILE $=$ ' C2H6. COF')

$\mathrm{N}=0$

$N$ N $=1$

$N W W=0$

GO TO 50

9 HRITE $(*, 208)$

208 FORMAT(' THE RANGE OF TEMPERATURE FOR PROPANE IS 85.47 TO $600 \mathrm{~K}$ '

$1 /{ }^{\prime}$ WITH PRESSURES TO $100 \mathrm{MPA}^{\prime}$ )

OPEN ( 5, FILE $=$ ' C 3 H8. COF')

$\mathrm{N}=0$

$\mathrm{NH}=1$

$\mathrm{NHW}=0$

GO TO 50

10 HRITE $(*, 209)$

209 FORMAT( ' THE RANGE OF TEMPERATURE FOR ISO BUTANE IS'

$1 / 113.55$ TO $600 \mathrm{~K}$

$1 /$ WITH PRESSURES TO $35 \mathrm{MPA}^{\prime}$ )

OPEN ( 5, FILE =' ISOB. COF')

$\mathrm{N}=0$

$N \mathrm{~N}=1$

$N$ WW $=0$

GO TO 50

$11 \operatorname{HRITE}(*, 210)$ 


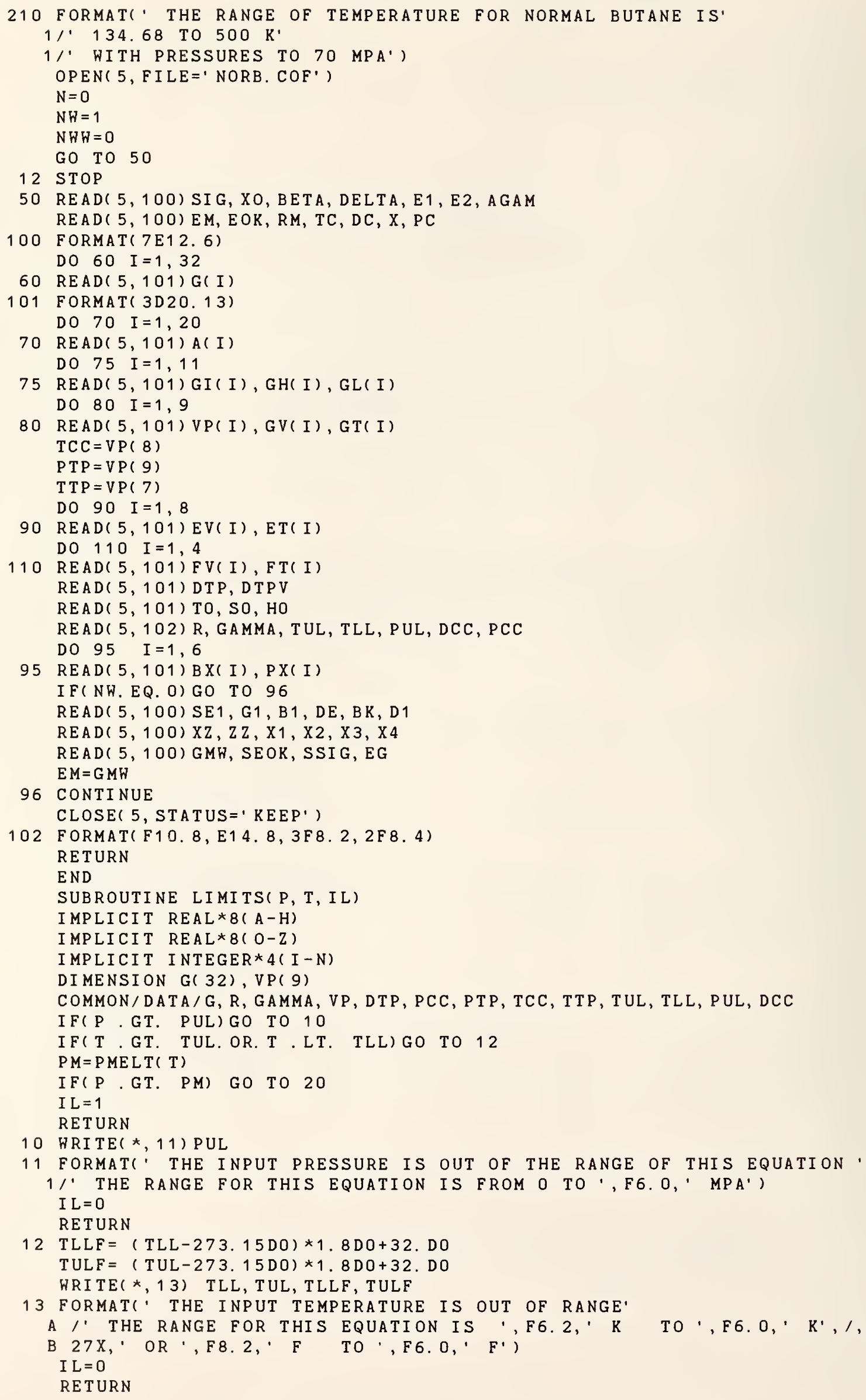


$20 \mathrm{TM}=\mathrm{TMELT}(\mathrm{P})$

$\mathrm{TF}=(\mathrm{TM}-273.15 \mathrm{D} 0) * 1.8 \mathrm{DO}+32 . \mathrm{DO}$

WRITE $(*, 22)$ TM, TF

22 FORMAT(' SOLID PHASE DETECTED. ',$/, '$ FOR THIS PRESSURE, TEMP'

$A^{\prime}$ SHOULD EXCEED ',F8. 3,' $\mathrm{K}$, OR', F9. 3,' $\mathrm{F}^{\prime}$ ')

I $L=0$

END

SUBROUTINE PROPS( PP, DD, TT, K)

IMPLICIT REAL*8( A-H)

I MPLICIT REAL*8( $0-Z)$

IMPLICIT INTEGER*4( I-N)

C THE 32 TERM EQUATION OF STATE, INPUT IS DENSITY(MOLES/L),

C TEMPERATURE( K), OUTPUT ( PP) IS PRESSURE(MPA), OR DP/DD IN

C LITER-MPA/MOLE OR DP/DT MPA/K OR $S, H, O R$ CV AT ONE LIMIT OF

C INTEGRATION

DIMENSI ON X( 33)

DIMENSION B( 33), G( 32$), \mathrm{VP}(9)$

EQUIVALENCE ( $B, X)$

COMMON/DATA/G, R, GAMMA, VP, DTP, PCC, PTP, TCC, TTP, TUL, TLL, PUL, DCC

DATA $M / 32 /$

$D=D D$

$P=P P$

$\mathrm{T}=\mathrm{T} \mathrm{T}$

$\mathrm{GM}=\mathrm{GAMMA}$

$\mathrm{D} 2=\mathrm{D} * \mathrm{D}$

$\mathrm{D} 3=\mathrm{D} 2 * \mathrm{D}$

$\mathrm{D} 4=\mathrm{D} 3 * \mathrm{D}$

$\mathrm{D} 5=\mathrm{D} 4 * \mathrm{D}$

$\mathrm{D} 6=\mathrm{D} 5 * \mathrm{D}$

$\mathrm{D} 7=\mathrm{D} 6 \star \mathrm{D}$

$\mathrm{D} 8=\mathrm{D} 7 * \mathrm{D}$

$\mathrm{D} 9=\mathrm{D} 8 * \mathrm{D}$

$\mathrm{D} 10=\mathrm{D} 9 * \mathrm{D}$

D $11=\mathrm{D} 10 * \mathrm{D}$

$\mathrm{D} 12=\mathrm{D} 11 * \mathrm{D}$

D $13=D 12 * D$

$\mathrm{TS}=\mathrm{DSQRT} \quad(\mathrm{T})$

$\mathrm{T} 2=\mathrm{T} \star \mathrm{T}$

$\mathrm{T} 3=\mathrm{T} 2 \star \mathrm{T}$

$\mathrm{T} 4=\mathrm{T} 3 * \mathrm{~T}$

$\mathrm{T} 5=\mathrm{T} 4 * \mathrm{~T}$

$\mathrm{F}=\mathrm{DEXP}(\mathrm{GM} * \mathrm{D} 2)$

C

GO TO $(100,200,300,400,500,600), \mathrm{K}$

ENTRY PRESS

$\mathrm{B}(1)=\mathrm{D} 2 * \mathrm{~T}$

$\mathrm{B}(2)=\mathrm{D} 2 * \mathrm{TS}$

$\mathrm{B}(3)=\mathrm{D} 2$

$\mathrm{B}(4)=\mathrm{D} 2 / \mathrm{T}$

$\mathrm{B}(5)=\mathrm{D} 2 / \mathrm{T} 2$

$\mathrm{B}(6)=\mathrm{D} 3 * \mathrm{~T}$

$B(7)=D 3$

$\mathrm{B}(8)=\mathrm{D} 3 / \mathrm{T}$

$\mathrm{B}(9)=\mathrm{D} 3 / \mathrm{T} 2$

$\mathrm{B}(10)=\mathrm{D} 4 \star \mathrm{T}$

$\mathrm{B}(11)=\mathrm{D} 4$

$\mathrm{B}(12)=\mathrm{D} 4 / \mathrm{T}$

$B(13)=D 5$

$\mathrm{B}(14)=\mathrm{D} 6 / \mathrm{T}$

$\mathrm{B}(15)=\mathrm{D} 6 / \mathrm{T} 2$

$\mathrm{B}(16)=\mathrm{D} 7 / \mathrm{T}$

$\mathrm{B}(17)=\mathrm{D} 8 / \mathrm{T}$

$\mathrm{B}(18)=\mathrm{D} 8 / \mathrm{T} 2$

$\mathrm{B}(19)=\mathrm{D} 9 / \mathrm{T} 2$

$\mathrm{B}(20)=\mathrm{D} 3 * \mathrm{~F} / \mathrm{T} 2$

$\mathrm{B}(21)=\mathrm{D} 3 * \mathrm{~F} / \mathrm{T} 3$

$\mathrm{B}(22)=\mathrm{D} 5 \star \mathrm{F} / \mathrm{T} 2$

$\mathrm{B}(23)=\mathrm{D} 5 \star \mathrm{F} / \mathrm{T} 4$

$\mathrm{B}(24)=\mathrm{D} 7 \star \mathrm{F} / \mathrm{T} 2$ 


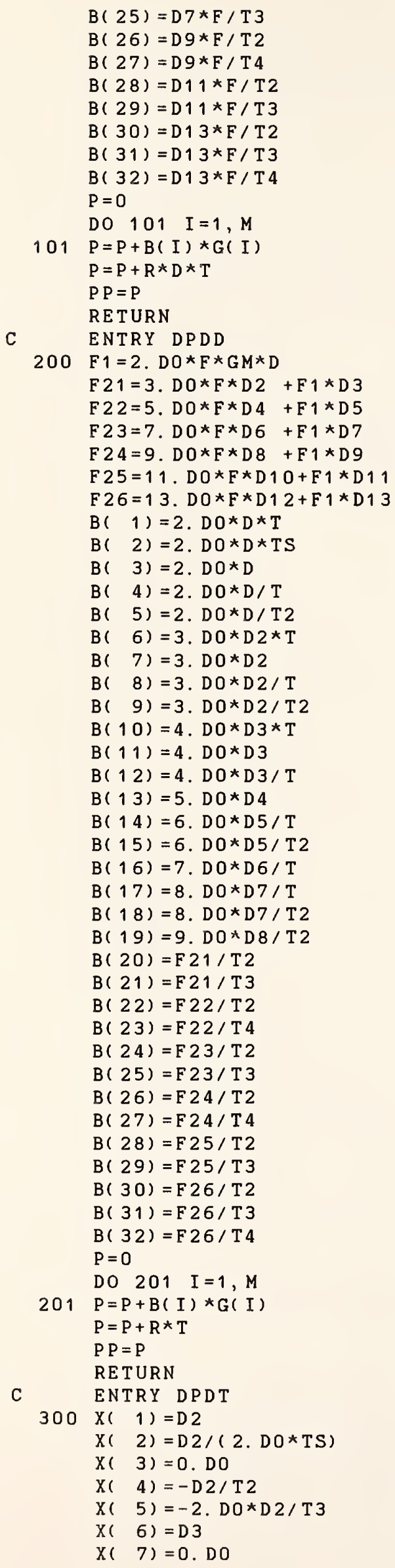




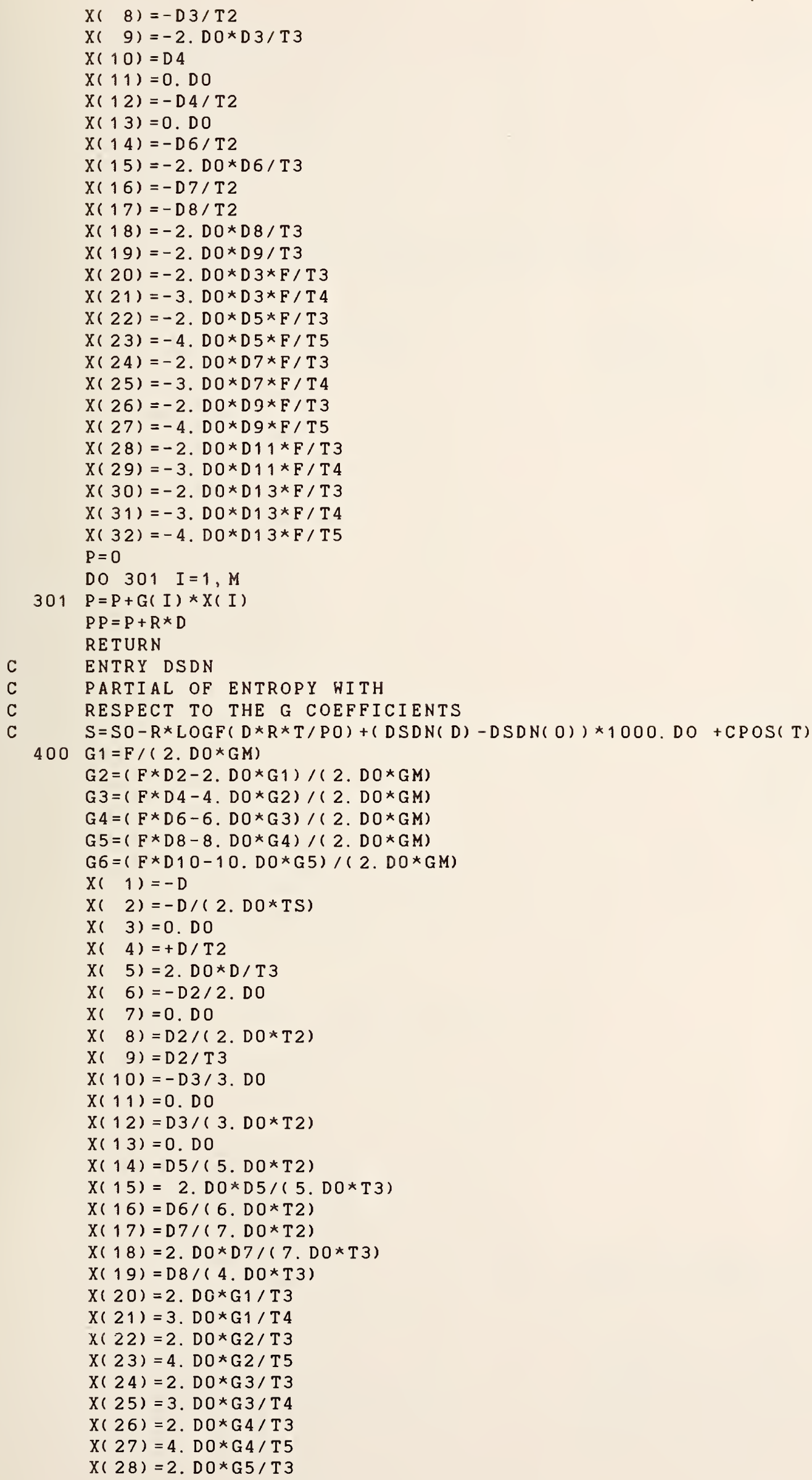




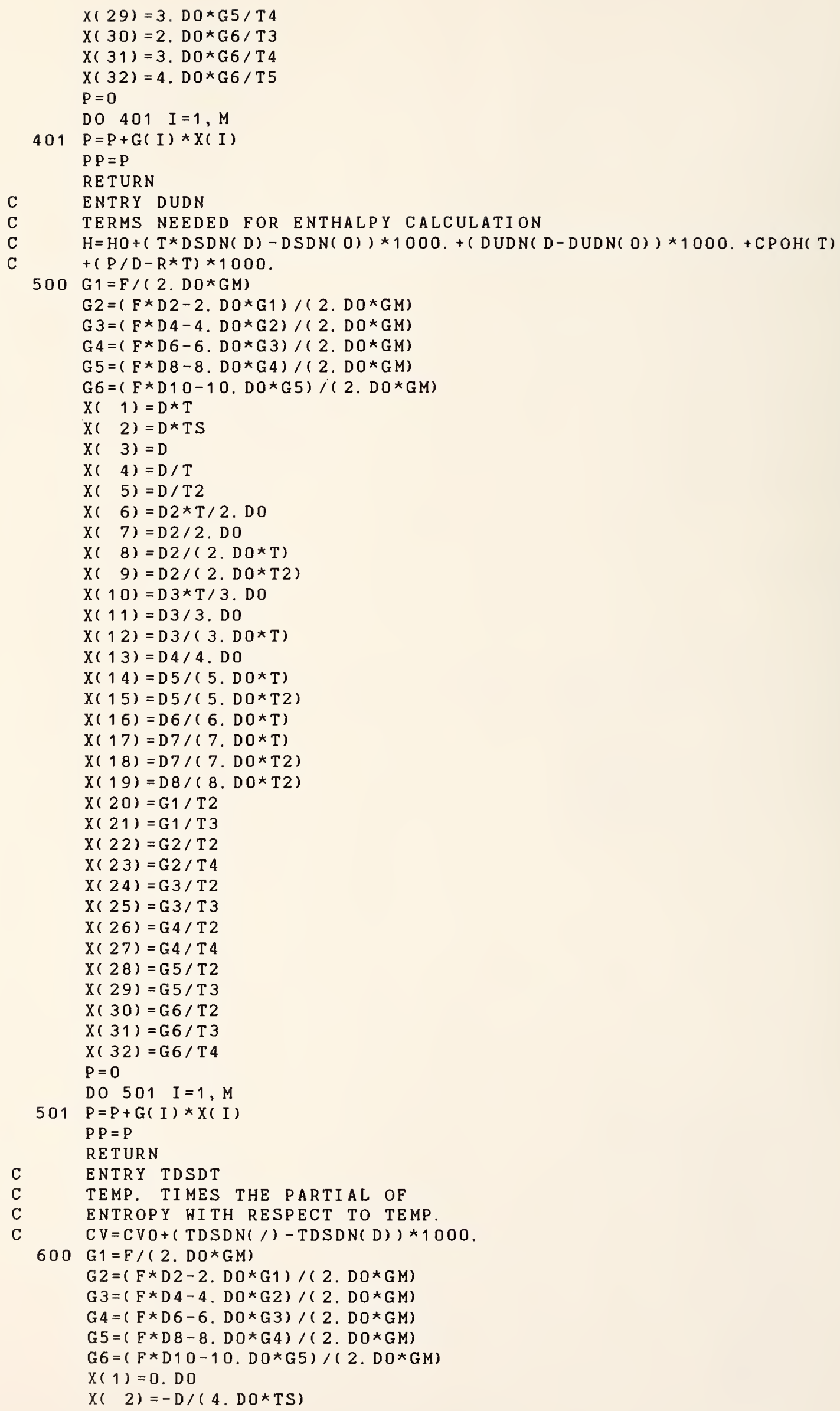


$X(3)=0 . D O$

$X(4)=2 . D 0 \star D / T 2$

$\mathrm{X}(5)=6 . \mathrm{DO} * \mathrm{D} / \mathrm{T} 3$

$X(6)=0 . D O$

$\mathrm{X}(7)=0 . \mathrm{DO}$

$\mathrm{X}(8)=\mathrm{D} 2 / \mathrm{T} 2$

$X(9)=3 . D 0 * D 2 / T 3$

$X(10)=0 . D O$

$\mathrm{X}(11)=0 . \mathrm{DO}$

$\mathrm{X}(12)=(2 . \mathrm{DO} \star \mathrm{D} 3) /(3 . \mathrm{D} 0 \star \mathrm{T} 2)$

$X(13)=0 . D 0$

$X(14)=(2 . D O * D 5) /(5 . D 0 * T 2)$

$X(15)=(6, D O \star D 5) /(5, D O * T 3)$

$\mathrm{X}(16)=\mathrm{D} 6 /(3 . \mathrm{D} 0 * \mathrm{~T} 2)$

$X(17)=(2 . D 0 * D 7) /(7 . D O \star T 2)$

$X(18)=(6 . D O \star D 7) /(7 . D O \star T 3)$

$X(19)=(3 . D 0 * D 8) /(4 . D 0 * T 3)$

$\mathrm{X}(20)=6 . \mathrm{DO} * \mathrm{G} 1 / \mathrm{T} 3$

$\mathrm{X}(21)=12 . \mathrm{DO} \star \mathrm{G} 1 / \mathrm{T} 4$

$X(22)=6 . D O * G 2 / \mathrm{T}^{3}$

$\mathrm{X}(23)=20 . \mathrm{DO} * \mathrm{G} 2 / \mathrm{T} 5$

$X(24)=6 . D 0 * G 3 / T 3$

$\mathrm{X}(25)=12 . \mathrm{DO} \star \mathrm{G} 3 / \mathrm{T} 4$

$\mathrm{X}(26)=6 . \mathrm{DO} * \mathrm{G} 4 / \mathrm{T} 3$

$\mathrm{X}(27)=20 . \mathrm{D} 0 * \mathrm{G} 4 / \mathrm{T} 5$

$X(28)=6 . D 0 * G 5 / T 3$

$\mathrm{X}(29)=12 . \mathrm{D} 0 * \mathrm{G} 5 / \mathrm{T} 4$

$\mathrm{X}(30)=6 . \mathrm{D} 0 * \mathrm{G} 6 / \mathrm{T} 3$

$\mathrm{X}(31)=12 . \mathrm{D} 0 * \mathrm{G} 6 / \mathrm{T} 4$

$\mathrm{X}(32)=20 . \mathrm{DO} \star \mathrm{G} 6 / \mathrm{T} 5$

$\mathrm{P}=0$

DO $601 \mathrm{I}=1, \mathrm{M}$

$601 P=P+G(I) * X(I)$

$\mathrm{PP}=\mathrm{P}$

END

DOUBLE PRECISION FUNCTION FINDTV(POBS)

IMPLICIT REAL*8( $A-H)$

IMPLICIT REAL*8( $0-\mathrm{Z})$

IMPLICIT INTEGER*4(I-N)

C ITERATES VAPOR PRESS EQN TO FIND TEMP(K), FOR INPUT OF PRESS(MPA).

C GIVEN AN INPUT PRESSURE( MPA)

COMMON/DATA/G, R, GAMMA, VP, DTP, PCC, PTP, TCC, TTP, TUL, TLL, PUL, DCC

DIMENSION G( 32 ), VP( 9 )

$\mathrm{T}=\mathrm{VP}(8)$

DO $7 \quad I=1,10$

$\mathrm{P}=\mathrm{VPN}(\mathrm{T})$

IF( DABS ( P-POBS) -. 000001DO*POBS) $8,8,6$

6 CONTINUE

$\mathrm{CORR}=(\mathrm{POBS}-\mathrm{P}) / \mathrm{DPDTVP}(\mathrm{T}, \mathrm{P})$

$7 \mathrm{~T}=\mathrm{T}+\mathrm{CORR}$

8 CONTINUE

FI NDT V $=$ T

END

DOUBLE PRECISION FUNCTION CV(D, T)

IMPLICIT REAL* $8(\mathrm{~A}-\mathrm{H})$

IMPLICIT REAL*8(O-Z)

IMPLICIT INTEGER* $4(\mathrm{I}-\mathrm{N})$

C CALCULATES CV $(\mathrm{J} ;(\mathrm{MOL} \star \mathrm{K}))$.

DATA R/8.31434DO/

$D D=D$

$\mathrm{T} T=\mathrm{T}$

CALL PROPS( CD, DD, TT, 6)

$\mathrm{DD}=0$. ODO

CALL PROPS( CO, DD, TT, 6)

$C V=C P I(T T, 1)+(C O-C D) * 1000 . D 0$

$\mathrm{CV}=\mathrm{C} \mathrm{V}-\mathrm{R}$

END

FUNCTION FINDD( P, T) 


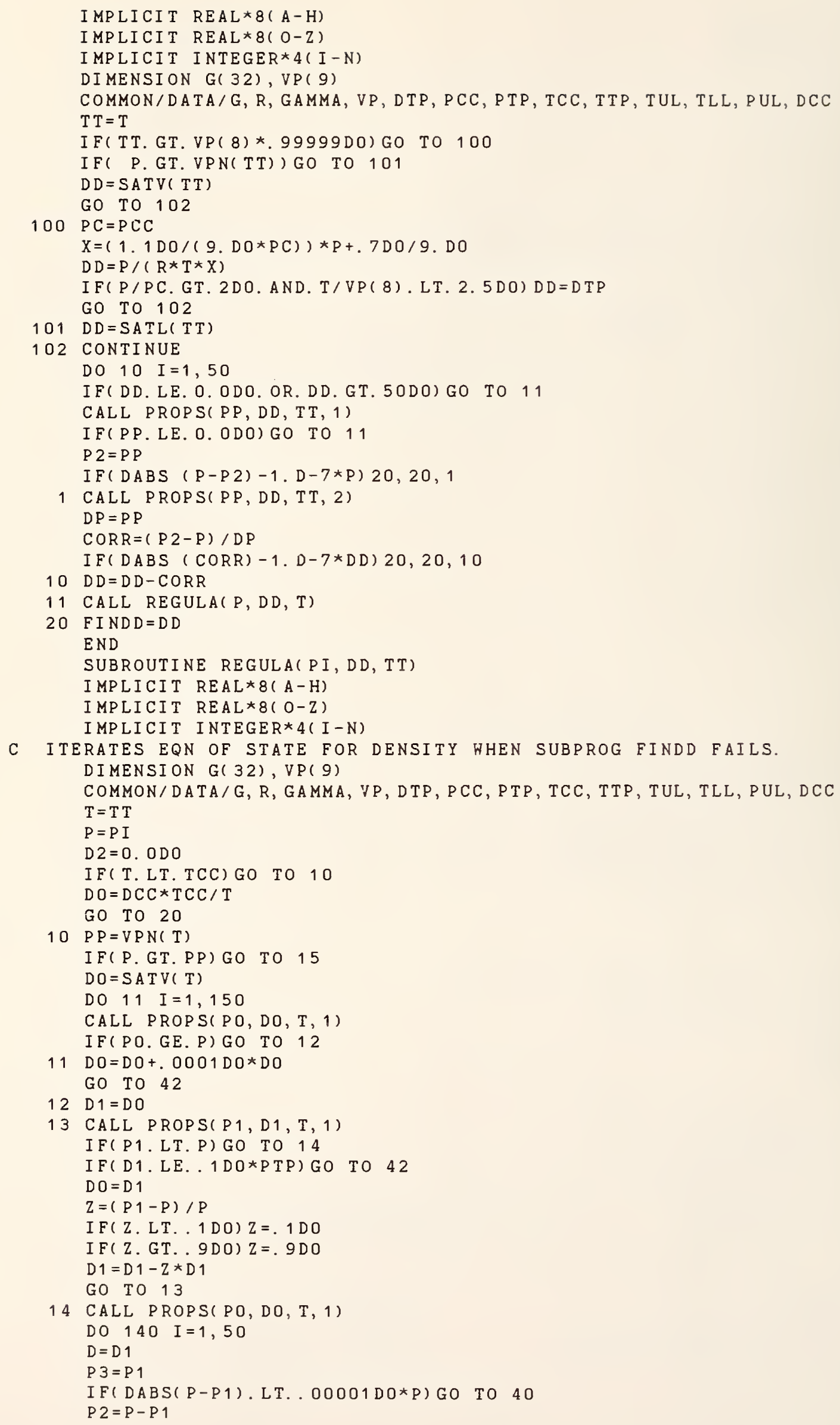




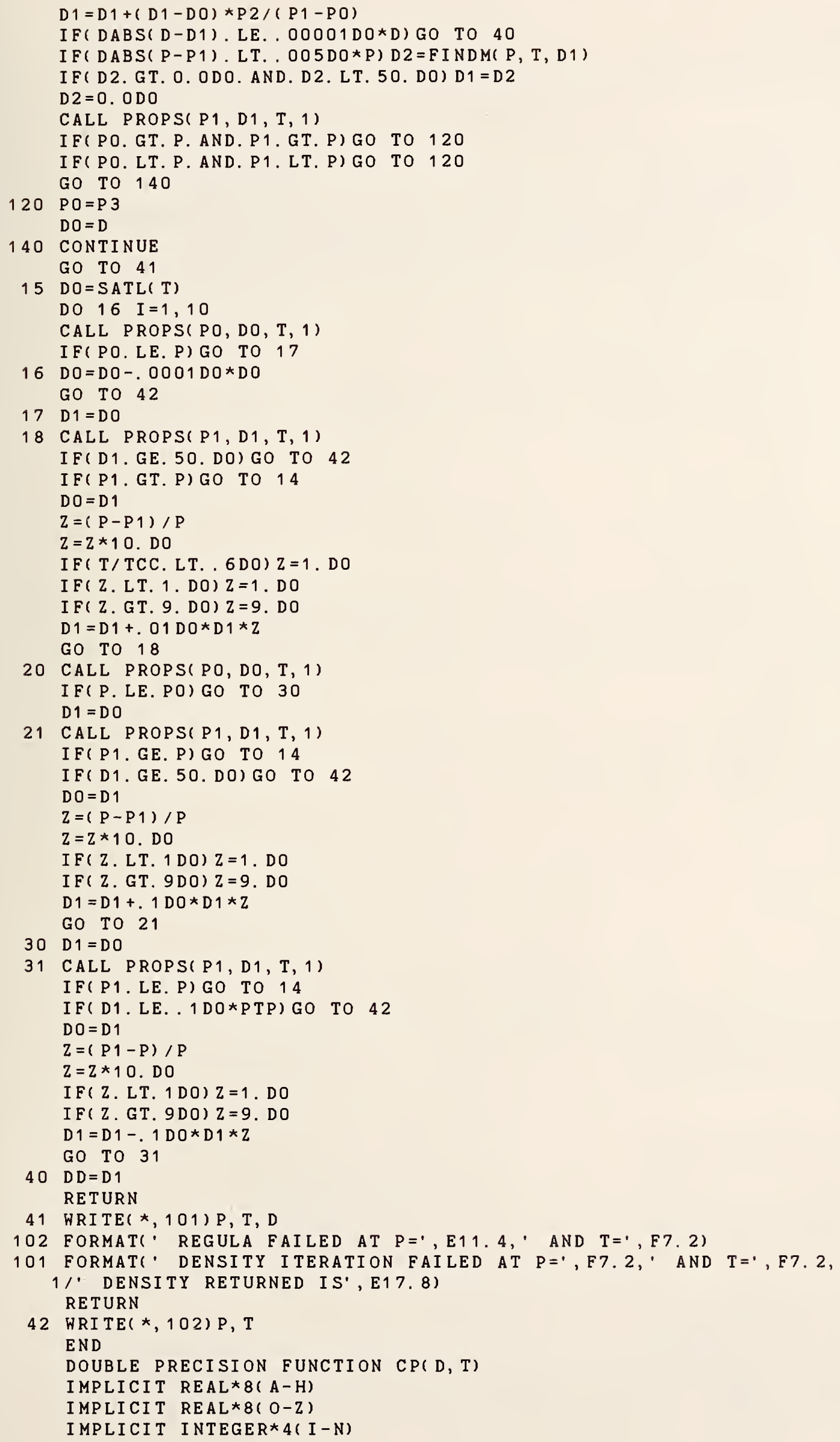


$C V E E=C V(D, T)$

CALL PROPS( DPT, D, T, 3)

$C A L L$ PROPS( DPD, D, T, 2)

$C P=C V E E+(T /(D \star \star 2) \star(D P T \star \star 2) / D P D) \star 1000 . D 0$

END

DOUBLE PRECISION FUNCTION DPDTVP(TT, P)

IMPLICIT REAL*8( $\mathrm{A}-\mathrm{H})$

I MPLICIT REAL*8( $0-Z)$

I MPLICIT INTEGER*4(I-N)

C CALCULATES THE DERIVATIVE OF PRESSURE WITH RESPECT TO TEMPERATURE

C AT SATURATION. INPUT IS TEMP(K), OUTPUT IS DPDT(MPA/K).

COMMON/DATA/G, R, GAMMA, VP, DTP, PCC, PTP, TCC, TTP, TUL, TLL, PUL, DCC DIMENSION $G(32)$, VP( 9$)$

$\mathrm{T}=\mathrm{T} \mathrm{T}$

IF(TT. GT. VP( 8)) GO TO 1

$X=(1 . D 0-V P(7) / T) /(1 . D O-V P(7) / V P(8))$

$\mathrm{DXDT}=(\mathrm{VP}(7) / \mathrm{T} * \star 2) /(1 . \mathrm{DO}-\mathrm{VP}(7) / \mathrm{VP}(8))$

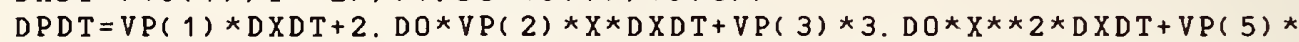

$1((1 . D O-X) * * V P(6)) * D X D T+V P(5) * X *((1 . D O-X) \star \star(V P(6)-1 . D 0)) * V P(6)$

$2 *(-D X D T)$

DPDTVP $=$ DPDT*P

RETURN

$1 \quad$ DPDTVP=0. ODO

END

DOUBLE PRECISION FUNCTION FINDM( $P, T, D D)$

I MPLICIT REAL* $8(\mathrm{~A}-\mathrm{H})$

I MPLICIT REAL* $8(0-Z)$

IMPLICIT INTEGER* $4(\mathrm{I}-\mathrm{N})$

C SOLVES FOR DENSITY(MOL/L) BY ITERATION. INPUT IS PRESSURE(MPA),

C TEMPERATURE( K), AND A STARTING VALUE OF DENSITY. THIS FCN IS AN

C ALTERNATIVE FOR FUNCTION FINDD.

$\mathrm{T} \mathrm{T}=\mathrm{T}$

DO $10 \quad I=1,50$

CALL PROPS( PP, DD, TT, 1)

$\mathrm{P} 2=\mathrm{P} P$

IF ( DABS ( P-P 2) -1. D-7*P) 20, 20,1

1 CALL PROPS( PP, DD, TT, 1)

$\mathrm{DP}=\mathrm{PP}$

$C O R R=(P 2-P) / D P$

$D=D D$

IF ( DABS (CORR) $-1 . D-7 * D) 20,20,10$

$10 \mathrm{DD}=\mathrm{DD}-\mathrm{CORR}$

$F$ I NDM $=0$

RETURN

20 FINDM=DD

END

DOUBLE PRECISION FUNCTION ENTHAL( $P, D, T)$

IMPLICIT REAL* $8(\mathrm{~A}-\mathrm{H})$

I MPLICI T REAL* $8(0-Z)$

IMPLICIT INTEGER* $4(\mathrm{I}-\mathrm{N})$

$R=.00831434 \mathrm{DO}$

$\mathrm{DD}=\mathrm{D}$

$\mathrm{T} T=\mathrm{T}$

CALL PROPS( SD, DD, TT, 4)

CALL PROPS( UD, DD, TT, 5)

$D D=0$. DO

CALL PROPS( SO, DD, TT, 4 )

CALL PROPS( UO, DD, TT, 5)

$E N T H A L=T \star(S D-S O) \star 1000 . D O+(U D-U 0) \star 1000 . D 0+C P I(T, 3)+(P / D-R * T) \star 1000 . D 0$ END

DOUBLE PRECISION FUNCTION ENTROP( D, T)

IMPLICIT REAL*8( $\mathrm{A}-\mathrm{H})$

I MPLICIT REAL* $8(0-Z)$

IMPLICIT INTEGER*4( I-N)

C

CALCULATES ENTROPY ( J/( MOL-K), FROM INPUT OF DENSITY(MOL/L) AND TEMP(K). $\mathrm{R}=.00831434 \mathrm{DO}$

$\mathrm{PO}=\quad .101325 \mathrm{DO}$

$\mathrm{D} D=\mathrm{D}$ 
$\mathrm{T} \mathrm{T}=\mathrm{T}$

CALL PROPS( SD, DD, TT, 4)

$\mathrm{DD}=0$

CALL PROPS( SO, DD, TT, 4)

$E N T R O P=(S D-S 0) \star 1000 . D 0-R * D L O G(D * R * T / P O) \star 1000 . D 0+C P I(T, 2)$

END

DOUBLE PRECISION FUNCTION SATL(TT)

IMPLICIT REAL*8( $\mathrm{A}-\mathrm{H})$

IMPLICIT REAL*8( $0-Z)$

IMPLICIT INTEGER*4( I-N)

C CALCULATES DENSITY(MOL/L) OF SATURATED LIQUID. INPUT IS TEMP(K).

DIMENSION A(20)

DIMENSION G( 32$), \operatorname{VP}(9)$

COMMON/DATA/G, R, GAMMA, VP, DTP, PCC, PTP, TCC, TTP, TUL, TLL, PUL, DCC

COMMON/SATC/A, DTPV, EG

COMMON/ISP/N, NW, NWW

IF(NH. EQ. 1) GO TO 30

$\mathrm{T}=\mathrm{TT}$

$\mathrm{K}=14$

$\mathrm{KK}=7$

10 IF(T.GE. TCC*. 99999DO) GO TO 20

I T T $=$ T C C

IF ( I TT+1-T. LT. 1. DO) $T=I T T$

$X=(T-T C C) /(T T P-T C C)$

$\mathrm{D}=\mathrm{A}(\mathrm{K}) \star \mathrm{DLOG}(\mathrm{X})$

DO $11 \mathrm{I}=2, \mathrm{KK}$

$\mathrm{K}=\mathrm{K}+1$

$M M=I$

I $F(M M . G E .5) M M=M M+1$

$11 D=D+A(K) *(1 . D O-X \star \star((M M-5) / 3 . D 0))$

IF(K. LT. 14) GO TO 12

$\mathrm{D}=\mathrm{DCC}+\mathrm{DEXP}(\mathrm{D})$ * $(\mathrm{DTP}-\mathrm{DCC})$

GO TO 13

$12 D=D C C+D E X P(D) \star(D T P V-D C C)$

13 SATL $=D$

IF ( I TT+1 - T T. LT. 1) SATL=D-(D-DCC) * $(T T-T)$

RETURN

20 SATL $=$ DCC

RETURN

30 CALL SSATL(DL, TT)

$S A T L=D L$

END

DOUBLE PRECISION FUNCTION SATV(TT)

IMPLICIT REAL*8( $\mathrm{A}-\mathrm{H})$

IMPLICIT REAL *8(0-Z)

IMPLICIT INTEGER* $4(I-N)$

C CALCULATES DENSITY(MOL/L) OF SATURATED VAPOUR. INPUT IS TEMP(K). DIMENSION A(20)

DIMENSION G( 32 ), VP( 9 )

COMMON/DATA/G, R, GAMMA, VP, DTP, PCC, PTP, TCC, TTP, TUL, TLL, PUL, DCC

COMMON/SATC/A, DTPV1, EG

COMMON/ISP/N, NW, NWW

IF ( NW. EQ. 1) GO TO 30

$\mathrm{K}=1$

$K K=13$

$T=T T$

10 IF (T.GE. TCC*. 99999DO) GO TO 20

I T T $=\mathrm{TCC}$

IF ( I T T +1 - T. LT. 1. DO) $T=I T T$

$X=(T-T C C) /(T T P-T C C)$

$\mathrm{D}=\mathrm{A}(\mathrm{K}) \star \mathrm{DLOG}(\mathrm{X})$

DO $11 \mathrm{I}=2, \mathrm{KK}$

$\mathrm{K}=\mathrm{K}+1$

$M M=I$

IF ( MM. GE. 5) $M M=M M+1$

$11 D=D+A(K) *(1 . D O-X * \star((M M-5) / 3 . D O))$

IF (K. LT. 14) GO TO 12

$D=D C C+D E X P(D) \star(D T P-D C C)$

GO TO 13 


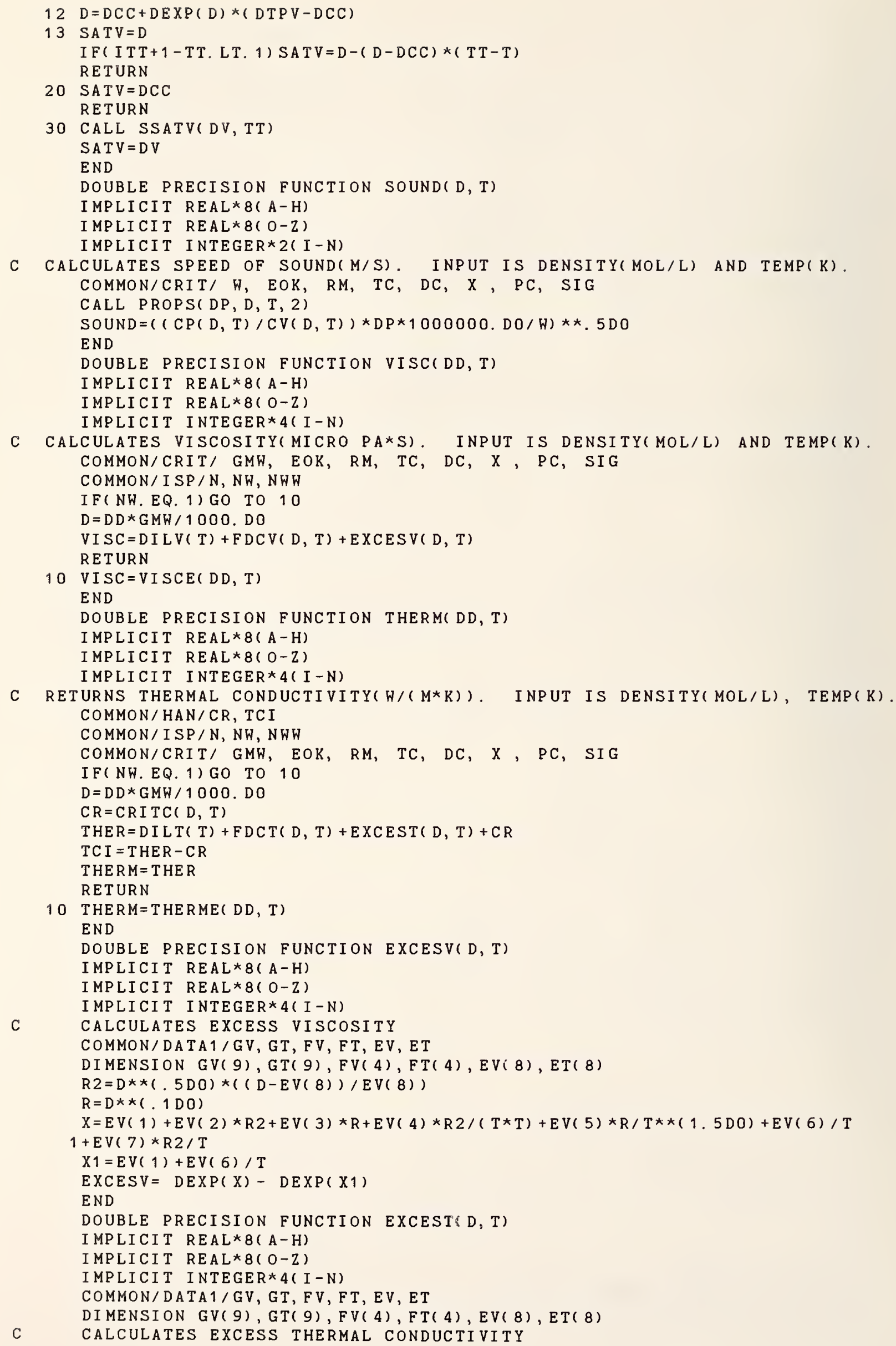




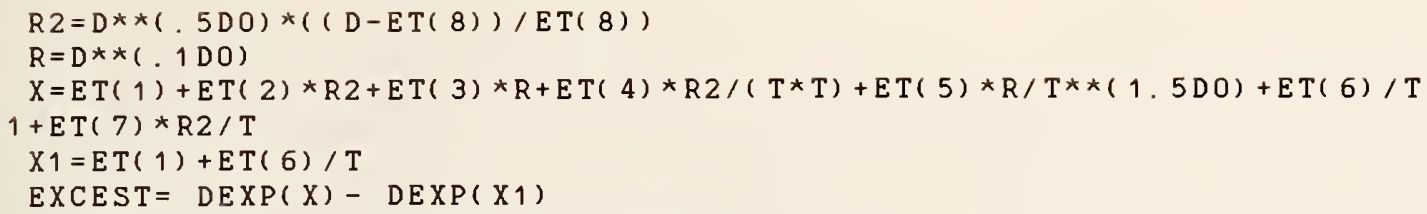




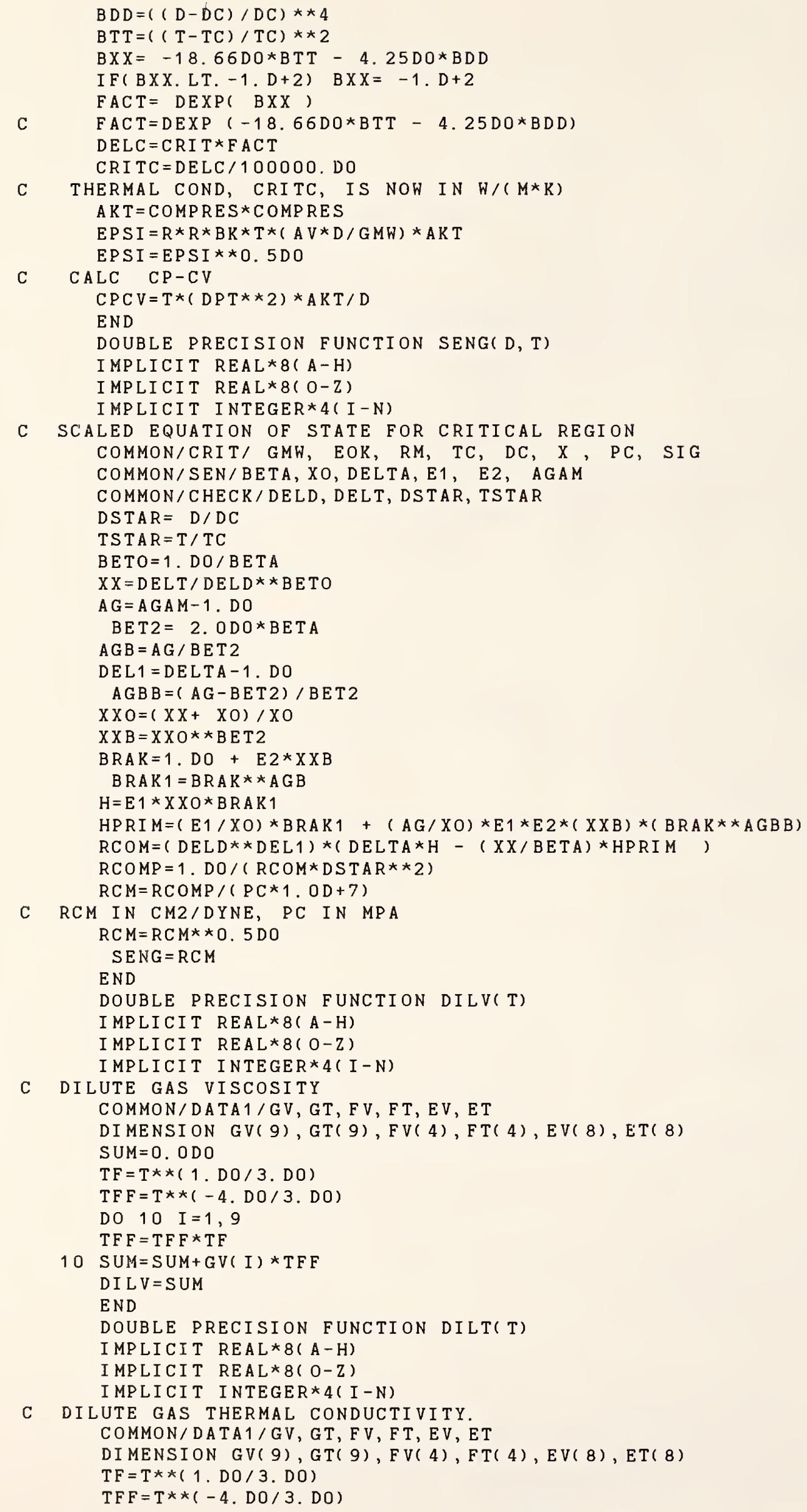


SUM $=0$

DO $20 \quad I=1,9$

$\mathrm{TFF}=\mathrm{TFF} * \mathrm{TF}$

$20 \mathrm{SUM}=\mathrm{SUM}+\mathrm{GT}(\mathrm{I}) \star \mathrm{TFF}$

DI L T $=$ SUM

END

DOUBLE PRECISION FUNCTION FINDP( D, T)

IMPLICIT REAL $8(\mathrm{~A}-\mathrm{H})$

I MPLICIT REAL*8(0-Z)

I MPLICI T INTEGER* $4(\mathrm{I}-\mathrm{N})$

DIMENSI ON G( 32$), V P(9)$

COMMON/DATA/G, R, GAMMA, VP, DTP, PCC, PTP, TCC, TTP, TUL, TLL, PUL, DCC

$\mathrm{DD}=\mathrm{D}$

$\mathrm{TT}=\mathrm{T}$

IF( TT. LT. TCC) GO TO 10

1 CALL PROPS( PP, DD, TT, 1)

F I NDP $=$ P P

RETURN

$10 \mathrm{P}=\mathrm{VPN}(\mathrm{TT})$

$\mathrm{DV}=\mathrm{F} I N D D(P-.0001 \mathrm{D} 0, \mathrm{TT})$

$D L=F I N D D(P+.0001 D 0, T T)$

IF( DD. LE. DV. OR. DD. GE. DL) GO TO 1

WRITE $(\star, 100) \mathrm{DV}, \mathrm{DL}, \mathrm{DD}$

CALL PROPS( PP, DV, TT, 1)

F I NDP $=$ P P

$D=D V$

100 FORMAT(' THE STATE POINT YOU HAVE SPECIFIED CORRESPONDS TO A.

$1 /$ DENSITY IN THE LIQUID VAPOR COEXISTENCE REGION'

$21^{\circ}$ THE DENSITY OF THE SATURATED VAPOR IS ', F6. $4,{ }^{\circ}$ MOLES/LITER'

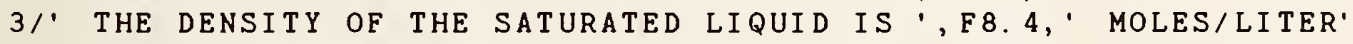

$4 / /^{\prime}$ AND THE INPUT DENSITY IS ',F8. 4, 'MOLES/LITER'

$5 /$ ' SATURATED VAPOR IS ASSUMED')

END

DOUBLE PRECISION FUNCTION FINDT( $P, D)$

I MPLICIT REAL* $8(A-H)$

I MPLICI T REAL* $8(0-Z)$

IMPLICIT INTEGER* 4 ( I-N)

C RETURNS TEMPERATURE( $K)$, FROM THE 32-TLRM MBNR EQN OF STATE.

C INPUT IS PRESSURE(MPA) AND DENSITY(MOL/L).

DI MENSION G( 32$), V P(9)$

COMMON/DATA/G, R, GAMMA, VP, DTP, PCC, PTP, TCC, TTP, TUL, TLL, PUL. DCC

$\mathrm{PP}=\mathrm{P}$

$\mathrm{DD}=\mathrm{D}$

IF ( P. GE. PCC) GO TO 1

TSAT $=$ FI NDT V ( PP)

$D V=F I N D D(P P-.00001 D 0, T S A T)$

$D L=F I N D D(P P+.0001 D 0, T S A T)$

IF(DD.GT. DV. AND. DD. LT. DL) GO TO 30

$\mathrm{TT}=\mathrm{T}$ S A T

GO TO 2

$1 \mathrm{TT}=\mathrm{TCC}$

2 DO $10 \quad I=1,10$

CALL PROPS( P2, DD, TT, 1)

I F ( DABS (PP-P2) -1. D-7 *PP) 20, 20, 11

11 CALL PROPS(DP, DD, TT, 3)

$C O R R=(P 2-P P) / D P$

IF ( DABS( CORR) - 1.D-5) 20,20, 10

$10 \mathrm{TT}=\mathrm{TT}-\mathrm{CORR}$

20 F I NDT $=$ T T

RETURN

$30 \mathrm{FINDT}=\mathrm{TSAT}$

$D=D V$

WRI TE $(*, 100) \mathrm{DV}, \mathrm{DL}, \mathrm{DD}$

100 FORMAT( ${ }^{\circ}$ THE STATE POINT YOU HAVE SPECIFIED CORRESPONDS TO'

$1 /{ }^{\prime}$ A DENSITY IN THE LIQUID VAPOR COEXISTENCE REGION'

$21^{\prime}$ DENSITY OF THE SATURATED VAPOR IS', F8. $4,^{\circ}$ MOLES/LITER'

3\% DENSITY OF THE SATURATED LIQUID IS', F8. 4, 'MOLES/LITER'

$4 /{ }^{\circ}$ INPUT DENSITY IS', F8. $4,^{\prime}$ MOLES/LITER'

$5 /{ }^{\circ}$ SATURATED VAPOR CONDITIONS ARE ASSUMED') 


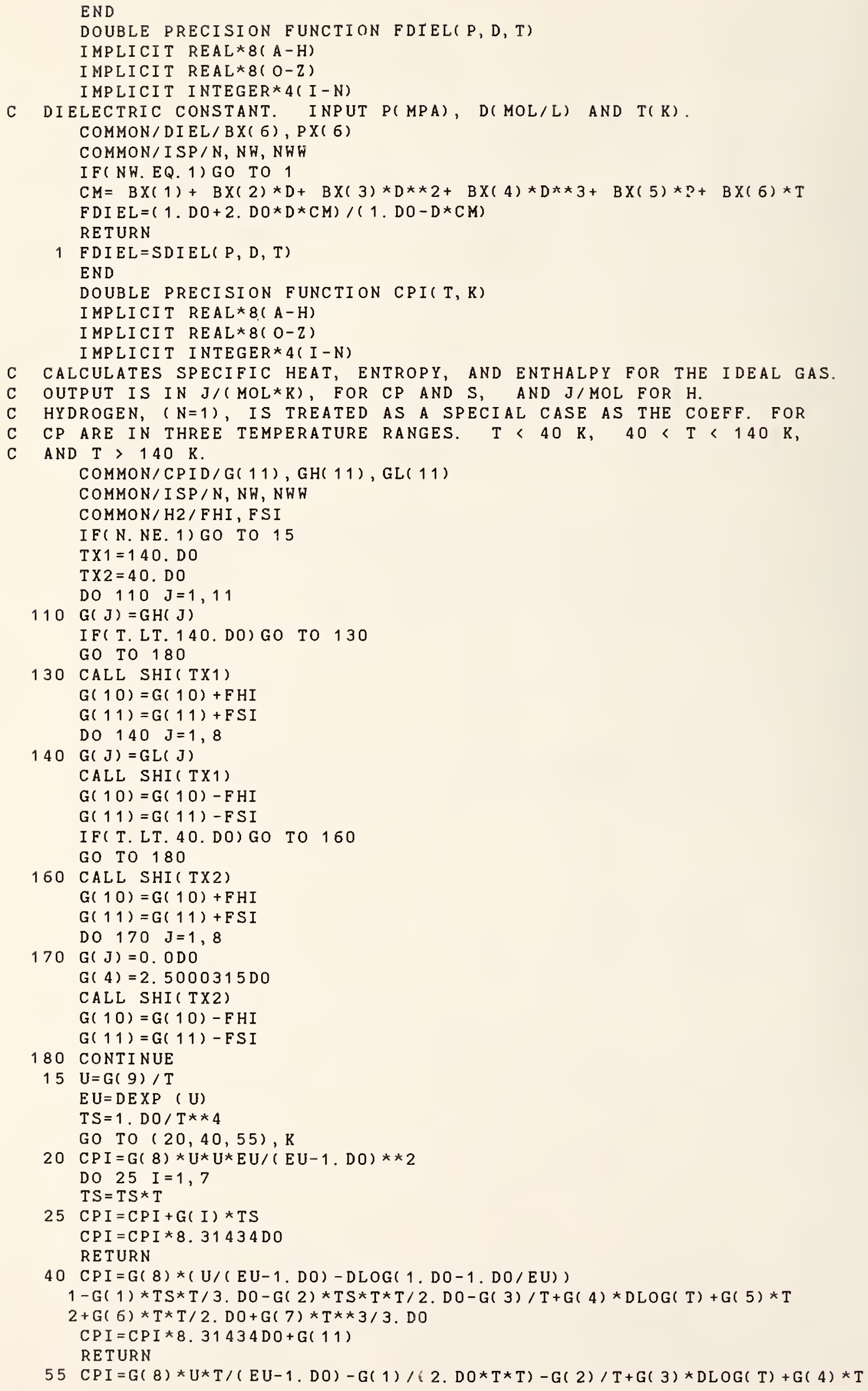


$1+\mathrm{G}(5) \star \mathrm{T} * \mathrm{~T} / 2 . \mathrm{DO}+\mathrm{G}(6) \star \mathrm{T} \star \star 3 / 3, \mathrm{DO}+\mathrm{G}(7) \star \mathrm{T} * \star 4 / 4 . \mathrm{DO}$

$C P I=C P I * 8.31434 D 0+G(10)$

END

SUBROUTINE SHI ( T)

I MPLICIT REAL*8( $\mathrm{A}-\mathrm{H})$

IMPLICIT REAL*8( $0-\mathrm{Z})$

I MPLICIT INTEGER* $4(\mathrm{I}-\mathrm{N})$

COMMON/CPID/G( 11$), \mathrm{GH}(11), \mathrm{GL}(11)$

COMMON/H2/F HI, FSI

$1 \mathrm{U}=\mathrm{G}(9) / \mathrm{T}$

$E U=D E X P(U)$

$\mathrm{GHI}=\mathrm{G}(8) \star \mathrm{U} * \mathrm{~T} /(\mathrm{EU}-1 . \mathrm{DO})-\mathrm{G}(1) /(2 . \mathrm{DO} * \mathrm{~T} * \mathrm{~T})-\mathrm{G}(2) / \mathrm{T}+\mathrm{G}(3) \star \mathrm{DLOG}(\mathrm{T})+\mathrm{G}(4) \star \mathrm{T}$

$A+G(5) * T * T / 2 . D O+G(6) \star T * \star 3 / 3 . D 0+G(7) \star T * \star 4 / 4 . D 0$

$\mathrm{FHI}=\mathrm{GHI} \star 8.31434 \mathrm{DO}$

$\mathrm{U}=\mathrm{G}(9) / \mathrm{T}$

$\mathrm{EU}=\mathrm{DEXP}(\mathrm{U})$

$\mathrm{TS}=1 . \mathrm{DO} / \mathrm{T} * * 4$

$\mathrm{GHS}=\mathrm{G}(8) \star(\mathrm{U} /(\mathrm{EU}-1 . \mathrm{DO})-\mathrm{DLOG}(1 . \mathrm{DO}-1 . \mathrm{DO} / \mathrm{EU}))-$

A $G(1) * T S * T / 3 . D O-G(2) * T S * T * T / 2 . D O-G(3) / T+G(4) * D L O G(T)+G(5) * T+$

B $G(6) * T * T / 2, D O+G(7) * T * \star 3 / 3 . D 0$

$\mathrm{FSI}=\mathrm{GHS} \star 8.31434 \mathrm{DO}$

END

DOUBLE PRECISION FUNCTION PMELT( T)

I MPLICIT REAL*8( A-H)

I MPLICIT REAL*8( $0-Z)$

IMPLICIT INTEGER* $4(I-N)$

C COMPUTES MELTING PRESSURE(MPA) FOR INPUT TEMPERATURE(K). COMMON/DIEL/BX(6), PX( 6)

COMMON/ISP/N, NW, NWW

I F(N. EQ. 1) GO TO 20

$10 \mathrm{PMELT}=\mathrm{PX}(1)+\mathrm{PX}(2) * \mathrm{~T} * \star \mathrm{PX}(3)$

RETURN

20 IF(T. LT. 22. DO) GO TO 10

$30 \mathrm{PMELT}=\mathrm{PX}(4)+\mathrm{PX}(5) * \mathrm{~T} * \star \mathrm{PX}(6)$

END

FUNCTION TMELT( P)

I MPLICIT REAL*8( $\mathrm{A}-\mathrm{H})$

IMPLICIT REAL*8(0-Z)

IMPLICIT INTEGER*4( I-N)

C

COMPUTES MELTING TEMPERATURE(K) FOR INPUT PRESSURE(MPA)

COMMON/DIEL/BX( 6), PX( 6)

COMMON/ISP/N, NW, NWW

I F( N. EQ. 1$)$ GO TO 20

$10 \operatorname{TMELT}=((n-\mathrm{PX}(1)) / \mathrm{PX}(2)) \star \star(1 . \mathrm{DO} / \mathrm{PX}(3))$

RETURN

20 IF( P. LT. 31.64DO) GO TO 10

$30 \mathrm{TMELT}=((\mathrm{P}-\mathrm{PX}(4)) / \mathrm{PX}(5)) \star \star(1 . \mathrm{DO} / \mathrm{PX}(6))$

END

DOUBLE PRECISION FUNCTION VPN(TT)

IMPLICIT REAL* $8(\mathrm{~A}-\mathrm{H})$

IMPLICI T REAL*8( $0-\mathrm{Z})$

IMPLICIT INTEGER*4(I-N)

CALCULATES VAPOR PRESSURE(MPA), INPUT IS TEMP(K).

DI MENSI ON G( 32 ), $V P(9)$

COMMON/DATA/G, R, GAMMA, VP, DTP, PCC, PTP, TCC, TTP, TUL, TLL, PUL, DCC $\mathrm{T}=\mathrm{T} \mathrm{T}$

$\mathrm{X}=(1 . \mathrm{D} 0-\mathrm{VP}(7) / \mathrm{T}) /(1 . \mathrm{D} 0-\mathrm{VP}(7) / \mathrm{VP}(8))$

$V P N=V P(9) \star D E X P(V P(1) \star X+V P(2) \star X \star X+V P(3) \star X \star \star 3+V P(4) \star X \star \star 4+V P(5) \star X \star$

$1(1 . D O-X) \star \star V P(6))$

END

SUBROUTINE SSATL( D1, T1)

IMPLICIT REAL*8( $\mathrm{A}-\mathrm{H})$

I MPLICIT REAL*8(O-Z)

IMPLICIT INTEGER*4(I-N)

SATURATED LIQUID AND VAPOR DENSITIES.

LIQUID DENSITIES ARE FROM R. D. MCCARTY.

VAPOR DENSITYES ARE FROM R. D. GOODWIN.

DIMENSION G( 32), VP( 9$), A(20)$

COMMON/DATA/G, R, GAMMA, VP, DTP, PCC, PTP, TCC, TTP, TUL, TLL, PUL, DCC 


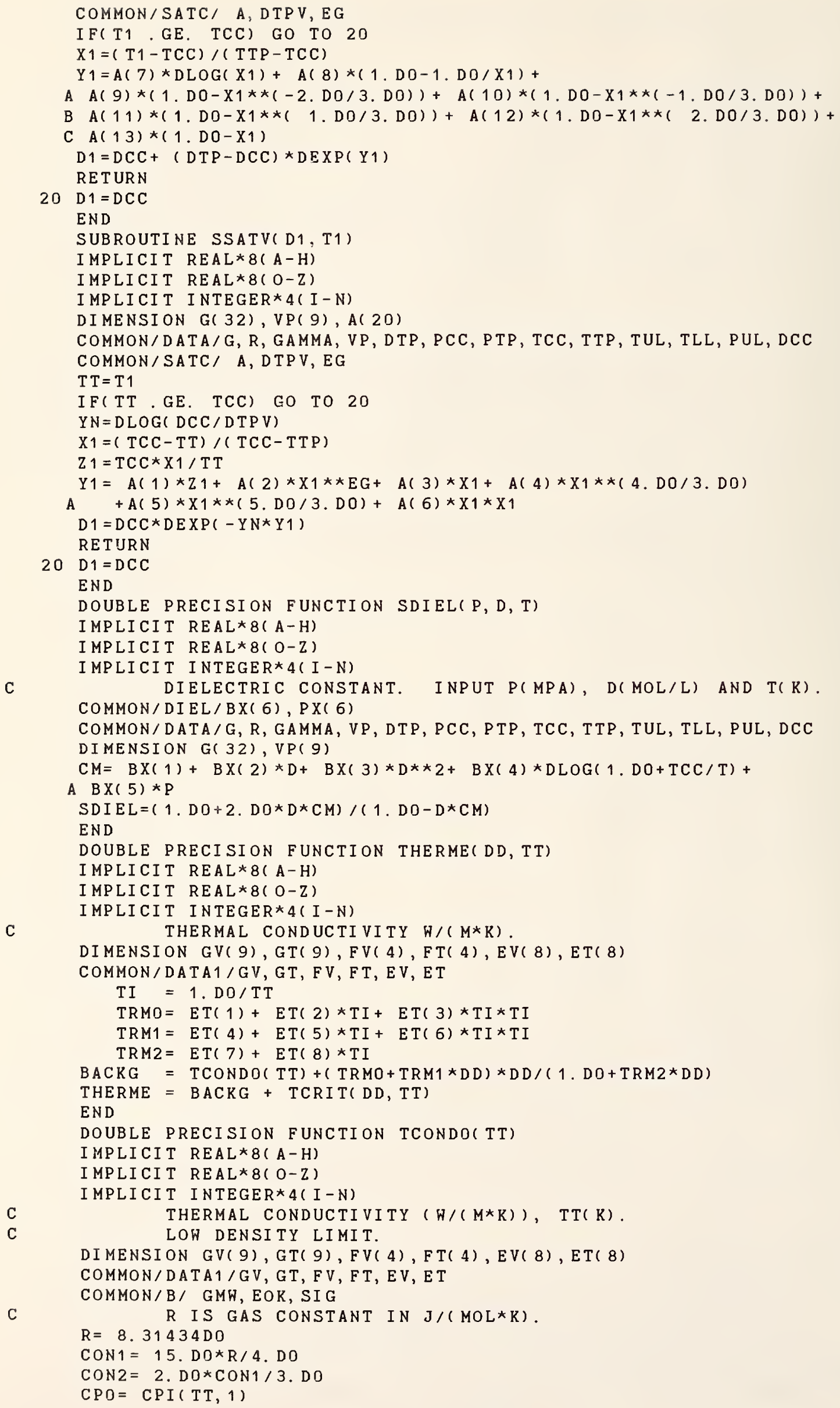


C

C

C

C

C

C

$0 \mathrm{BGAM}=\mathrm{XZ} \star \star \mathrm{G} 1 / \mathrm{D} 1 \star((1 . \mathrm{D} 0+\mathrm{E} 1) / \mathrm{E} 1) \star \star((\mathrm{G} 1-1 . \mathrm{D} 0) /(2 . \mathrm{D} 0 \star \mathrm{B} 1))$

CHISTAR=BGAM* $(D E L T) \star \star(-G 1)$

GO TO 50

20 IF(DELD. LE. 0.25DO. AND. DELT. LT. 0.03DO) GO TO 30 GO TO 40

C

$\mathrm{Y}=(\mathrm{XX}+\mathrm{XZ}) / \mathrm{XZ}$

$\mathrm{TOP}=\mathrm{DELD} \star \star *(-\mathrm{G} 1 / \mathrm{B} 1) \star((1 . \mathrm{DO}+\mathrm{E} 1) /(1 . \mathrm{DO}+\mathrm{E} 1 * \mathrm{Y} \star \star(2 . \mathrm{D} 0 * \mathrm{~B} 1))) \star \star((\mathrm{G} 1-1 . \mathrm{D} 0$

$1) /(2, D O * B 1))$

$D I V=D 1 *(D E+(Y-1 . D 0) *(D E-1 . D 0 / B 1+E 1 * Y \star \star(2 . D 0 * B 1)) /(1 . D 0+E 1 * Y * \star(2$.

$1 D 0 * B 1))$ )

CHISTAR=TOP $/$ DI V

GO TO 50

C

40 CHISTAR $=$

$50 \mathrm{CHI}=\mathrm{CHISTAR} \star \star X 3$

$\mathrm{UPPER}=\mathrm{X} 4$ * $\mathrm{BK} / \mathrm{PC} 1 \star(\mathrm{TT}$ * DPT $/ \mathrm{RSTAR}) \star \star 2 \star \mathrm{CHI} \star \mathrm{DFACT}$

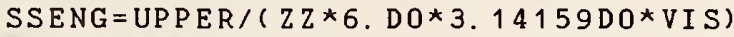

TCRI T $=$ SSENG

END

DOUBLE PRECISION FUNCTION VISCE(DS, TS)

IMPLICIT REAL* $8(\mathrm{~A}-\mathrm{H})$

IMPLICIT REAL* $8(0-\mathrm{Z})$

IMPLICIT INTEGER*4( I-N)

DIMENSION G( 32$), V P(9)$

DIMENSION GV( 9), GT( 9), FV( 4), $\mathrm{FT}(4), \operatorname{EV}(8), \operatorname{ET}(8)$

COMMON/DATA1/GV, GT, FV, FT, EV, ET

COMMON/DATA/G, R, GAMMA, VP, DTP, PCC, PTP, TCC, TTP, TUL, TLL, PUL, DCC

ETAO $=\operatorname{VSCTYO}(\mathrm{TS}, \mathrm{DS})$

$\mathrm{TRM} 1=\mathrm{EV}(3)+\mathrm{EV}(4) \star \mathrm{TS} \star \star(-3 . \mathrm{DO} / 2 . \mathrm{DO})$

$\mathrm{TRM} 2=\mathrm{EV}(5)+\mathrm{EV}(6) / \mathrm{TS}+\mathrm{EV}(7) \star \mathrm{TS} \star \star(-2 . \mathrm{D} 0)$

TRMX $=\operatorname{DEXP}(\mathrm{EV}(1)+\mathrm{EV}(2) / \mathrm{TS})$

$\mathrm{R} 1=\mathrm{DS} \star 0.1 \mathrm{DO}$

$\mathrm{R} 2=((\mathrm{DS}-\mathrm{DCC}) / \mathrm{DCC}) \star \mathrm{DS} \star \star 0.5 \mathrm{D} 0$

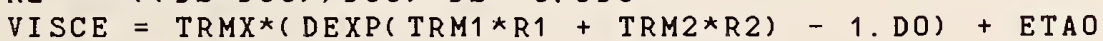




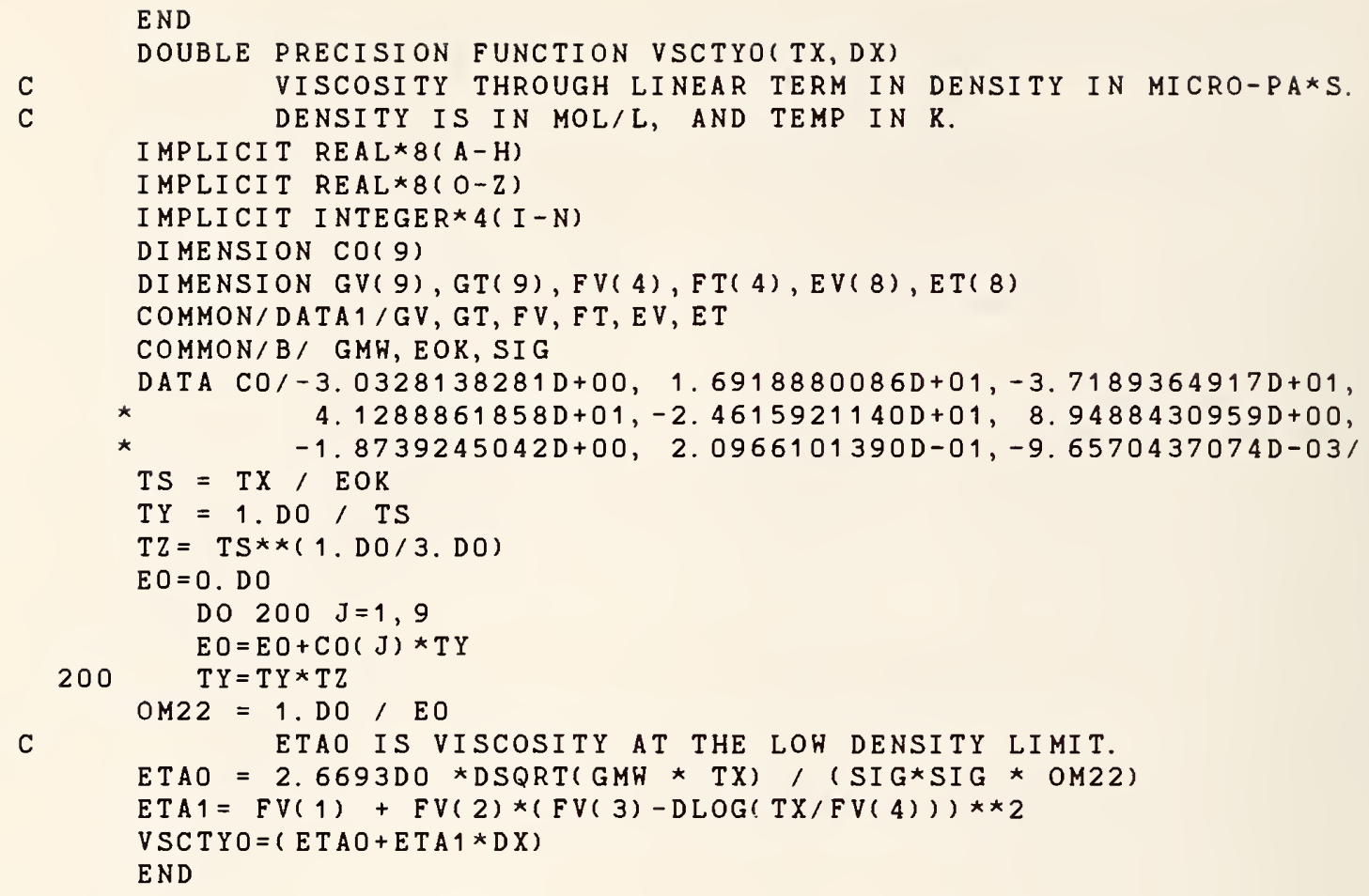


ARGON. COF

$.329700 E+01.183000 E+00.355000 E+00.435200 E+01.227000 E+01.287000 E+00.11$ $9000 E+01$

$.399480 E+02.152800 E+03.366900 E-07.150725 E+03.533000 E+00.171240 E+01.486190$ $\mathrm{E}+01$

-. $6569731294000 \mathrm{D}-04$

. $1822957801000 \mathrm{D}-01$

-. $3649470141000 D+00$

. $1232012107000 D+02$

$-.8613578274000 D+03$

$7978579691000 D-05$

-. $2911489110000 D-02$

$7581821758000 D+00$

$8780488169000 D+03$

. $1423145989000 D-07$

. $1674146131000 D-03$

-. $3200447909000 D-01$

. $2561766372000 D-05$

-. $5475934941000 \mathrm{D}-04$

-. $4505032058000 \mathrm{D}-01$ $.2013254653000 D-05$

-. $1678941273000 D-07$ . $4207329271000 D-04$

-. $5444212996000 D-06$

-. $8004855011000 D+03$

$-.1319304201000 D+05$

-. $4954923930000 D+01$ . $8092132177000 D+04$

-. $9870104061000 D-02$ . $2020441562000 D+00$

-. $1637417205000 D-04$

-. $7038944136000 \mathrm{D}-01$

-. $1154324539000 D-07$ . $1555990117000 \mathrm{D}-05$

-. $1492178536000 D-10$

$-.1001356071000 D-08$ . $2933963216000 D-07$

-. $2702629237770 D+02$ . $1310402418660 D+00$

-. $2674864381280 D+01$ . $3001768044060 D+02$

$-.8758991493260 \mathrm{D}+02$

-. $4082674364560 \mathrm{D}+02$ . $1042680664510 \mathrm{D}+03$

$-.6712785553790 D+02$ $.1510029357010 D+02$

-. $3312435366370 D+02$ $.6331462125810 D+02$

-. $4271497068990 D+02$ . $1005999000300 D+02$ . $1376820849000 D+02$

$-.6646303631910 D-01$ . $1333687827300 D+01$

-. $1443714632440 D+02$ $.6019384720000 D+02$

-. $2308884638870 D+02$ . $4653183588870 D+01$

0 .

$0 . \quad 0$

$0 . \quad 0$

0 . 0 .

$2500000000000 D+010$

0 .

0.20

0.

0.10

o. $2500000000000 D+01$

0

0.

$0 . \quad 0$.

0. 
-. $8011774999613 \mathrm{D}+000$. . $3630410930969 D+020$.

. $3415111551900 \mathrm{D}+01$

. $1191081251900 \mathrm{D}+01$

-. $3407632334000 \mathrm{D}+00$

0 .

$.8955585525100 \mathrm{D}+00$

. $1500000000000 \mathrm{D}+01$

$.8380000000000 D+02$

. $1508600000000 \mathrm{D}+03$

. $6890606625000 \mathrm{D}-01$

-. $1231357908600 \mathrm{D}+02$

$.2069468571200 \mathrm{D}+00$

. $1602914512200 \mathrm{D}+02$

$.1171746135100 \mathrm{D}+04$

-. $5699589878000 \mathrm{D}+03$

$4013607193300 \mathrm{D}+02$

. $3987012240300 D+02$

$5370000000000 \mathrm{D}+00$

. $1465365243300 \mathrm{D}+00$

-. $7748742496500 \mathrm{D}-01$

$1400000000000 D+01$

$1528000000000 \mathrm{D}+03$

$3540027619188 \mathrm{D}+02$

$2981500000000 \mathrm{D}+03$

$.00831434-.55542372 \mathrm{E}-02$

$.6114547278700 \mathrm{D}+04$

$-.1039439031200 \mathrm{D}+05$

. $6759461461900 \mathrm{D}+04$

$-.2253650938000 \mathrm{D}+04$ $4259395013800 \mathrm{D}+03$

-. $4725267109300 \mathrm{D}+02$ . $3179527542500 \mathrm{D}+01$

-. $1162908378000 \mathrm{D}+00$

$.1804301059200 \mathrm{D}-02$

-. $3332702733200 D+02$

0 .

. $3069485997100 D+02$

0 .

$2295655167400 \mathrm{D}+04$

-. $3555941584800 D+03$

0 .

$1000000000000 \mathrm{D}+01$

$.2414210327000 \mathrm{D}-01$

. $7569623425500 \mathrm{D}-02$

. $1000000000000 D+01$

. $1528000000000 \mathrm{D}+03$

$1029227022329 \mathrm{D}+00$

$1547335000000 \mathrm{D}+03$

0 .

0 .

0 .

0 .

0 .

0 .

PH2. COF

0 .

0 .

. $201594 \mathrm{E}+010$.

. $4675528393416 \mathrm{D}-04$

. $4289274251454 \mathrm{D}-02$

-. $5164085596504 \mathrm{D}-01$ $2961790279801 \mathrm{D}+00$

-. $3027194968412 \mathrm{D}+01$

. $1908100320379 \mathrm{D}-05$

-. $1339776859288 \mathrm{D}-03$

. $3056473115421 \mathrm{D}-01$

$5161197159532 \mathrm{D}+01$

$1999981550224 \mathrm{D}-07$

$2896367059356 \mathrm{D}-04$

-. $2257803939041 \mathrm{D}-02$

-. $2287392761826 \mathrm{D}-06$

. $2446261478645 \mathrm{D}-05$

-. $1718181601119 \mathrm{D}-03$

-. $5465142603459 \mathrm{D}-07$ $4051941401315 \mathrm{D}-09$ $1157595123961 \mathrm{D}-06$

-. $1269162728389 D-08$

-. $4983023605519 \mathrm{D}+01$

-. $1606676092098 \mathrm{D}+02$

-. $1926799185310 \mathrm{D}-01$

. $9319894638928 \mathrm{D}+00$

-. $3222596554434 \mathrm{D}-04$

. $1206839307669 \mathrm{D}-03$

-. $3841588197470 \mathrm{D}-07$

-. $4036157453608 \mathrm{D}-05$

-. $1250868123513 \mathrm{D}-10$ $1976107321888 \mathrm{D}-09$

-. $2411883474011 \mathrm{D}-13$

-. $4127551498251 \mathrm{D}-13$
-. $2105621650000 \mathrm{D}+03$

$.1777605270000 \mathrm{D}+00$

. $1598178680000 \mathrm{D}+01$

0 .

0 .

0 .
0.

0 .

$.6277770374200 \mathrm{D}+01$

$-.9609637663700 \mathrm{D}+01$

$5888754919100 \mathrm{D}+01$

-. $1892092632000 \mathrm{D}+01$

$3488657143700 \mathrm{D}+00$

-. $3801678619300 \mathrm{D}-01$

$.2520728316700 \mathrm{D}-02$

-. $9109874447800 \mathrm{D}-04$

. $1399084294200 \mathrm{D}-05$

$.6196500000000 \mathrm{D}+04$

$100.00 \quad 13.4100 \quad 4.9058$
0 .
0 .
0 .

0 .

0 .

0 .

0.

0.

. $171240 \mathrm{E}+010$. 
. $8917972883610 \mathrm{D}-12$

. $9166177201870 \mathrm{D}+02$

-. $1794925244460 \mathrm{D}+00$ $.4546711583950 \mathrm{D}+01$

-. $6584995897880 \mathrm{D}+02$ . $7344668045350 D+03$

-. $6825010451750 D+03$ $.6317836747100 D+03$

-. $5394088732820 \mathrm{D}+03$ $4309238117830 D+03$

-. $3002957388110 D+03$ $1565671653460 \mathrm{D}+03$

-. $5041036082250 D+02$ $.7207069265140 \mathrm{D}+01$

-. $1239444403180 \mathrm{D}+03$ $1403348001420 \mathrm{D}+01$

-. $2110238043130 D+02$ . $1732546228170 \mathrm{D}+03$

-. $4442945808710 \mathrm{D}+03$

$.1386993653550 \mathrm{D}+03$

-. $2357741610150 \mathrm{D}+02$ . $5262185164597 \mathrm{D}+08$

$-.1487906248823 \mathrm{D}+07$ $.1601391392264 \mathrm{D}+05$

-. $8031235938946 \mathrm{D}+02$

$.2307407941873 \mathrm{D}+00$

-. $3176386248370 \mathrm{D}-03$ $1643857271214 \mathrm{D}-06$ $.9230816464058 \mathrm{D}+01$ $3000000000000 D+04$

$-.6547883783722 \mathrm{D}+06$

0 .

$3053001341640 \mathrm{D}+010$ $2808109258130 \mathrm{D}+010$.

$-.6554612165670 \mathrm{D}+000$.

0 .

$1595144393740 D+01$ $1581445442800 \mathrm{D}+0$ $1380000000000 \mathrm{D}+020$ $3293800000000 \mathrm{D}+020$.

$7042087500000 \mathrm{D}-020$.

0 .

0 .

0 .

0 .

0 .

0 .

0.

0 .

0 .

0 .

0 .

0 . 0 . $3821428945438 \mathrm{D}+02$

$00831434-.41000000 \mathrm{E}-0$ $2024544300000 D-02$ $3717183200000 \mathrm{D}-06$

$-.9208501300000 D-08$

-. $3406532800000 D-11$

0 .

0 .
$.5262185164597 \mathrm{D}+08$

-. $1487906248823 \mathrm{D}+07$ $.1601391392264 \mathrm{D}+05$

$-.8031235938946 \mathrm{D}+02$ $.2307407941873 \mathrm{D}+00$

-. $3176386248370 \mathrm{D}-03$ $1643857271214 \mathrm{D}-06$ . $9230816464058 \mathrm{D}+01$ $3000000000000 D+04$

$-.6547883783722 \mathrm{D}+06$ $3850717781962 \mathrm{D}+04$ .

0 .

0 .

0 . .

0 .

0 .

0 .

0 .

0 .

0 .

0 .

0 .

0 .

0 .

0. 0.

$6322296353698 \mathrm{D}-01$

$\begin{array}{lll}02 & 400.00 \quad 13.80\end{array}$ $.2128148439500 D+02$ . $1257466430000 D+00$ . $1955000000000 \mathrm{D}+01$

$-.2652891150000 D+02$ $.2485785960000 \mathrm{D}+00$ . $1764739000000 \mathrm{D}+01$
$.2905965792270 D+06$

$-.2831103639248 D+05$

. $1050424877391 \mathrm{D}+04$

-. $1535751501769 \mathrm{D}+02$

. $1218941696566 \mathrm{D}+00$

$-.2599406479908 \mathrm{D}-04$

-. $1288757333406 \mathrm{D}-05$

$.1717441975231 \mathrm{D}+06$

. $3000000000000 \mathrm{D}+04$

$-.6547883783722 \mathrm{D}+06$

. $3850717781962 \mathrm{D}+04$

0 .

0 .

0 .

0 .

0 .

0 .

0 .

0 .

0 .

0 .

$\begin{array}{llll}120.00 & 15.5560 & 1.2838\end{array}$ 
N2. $\mathrm{COF}$

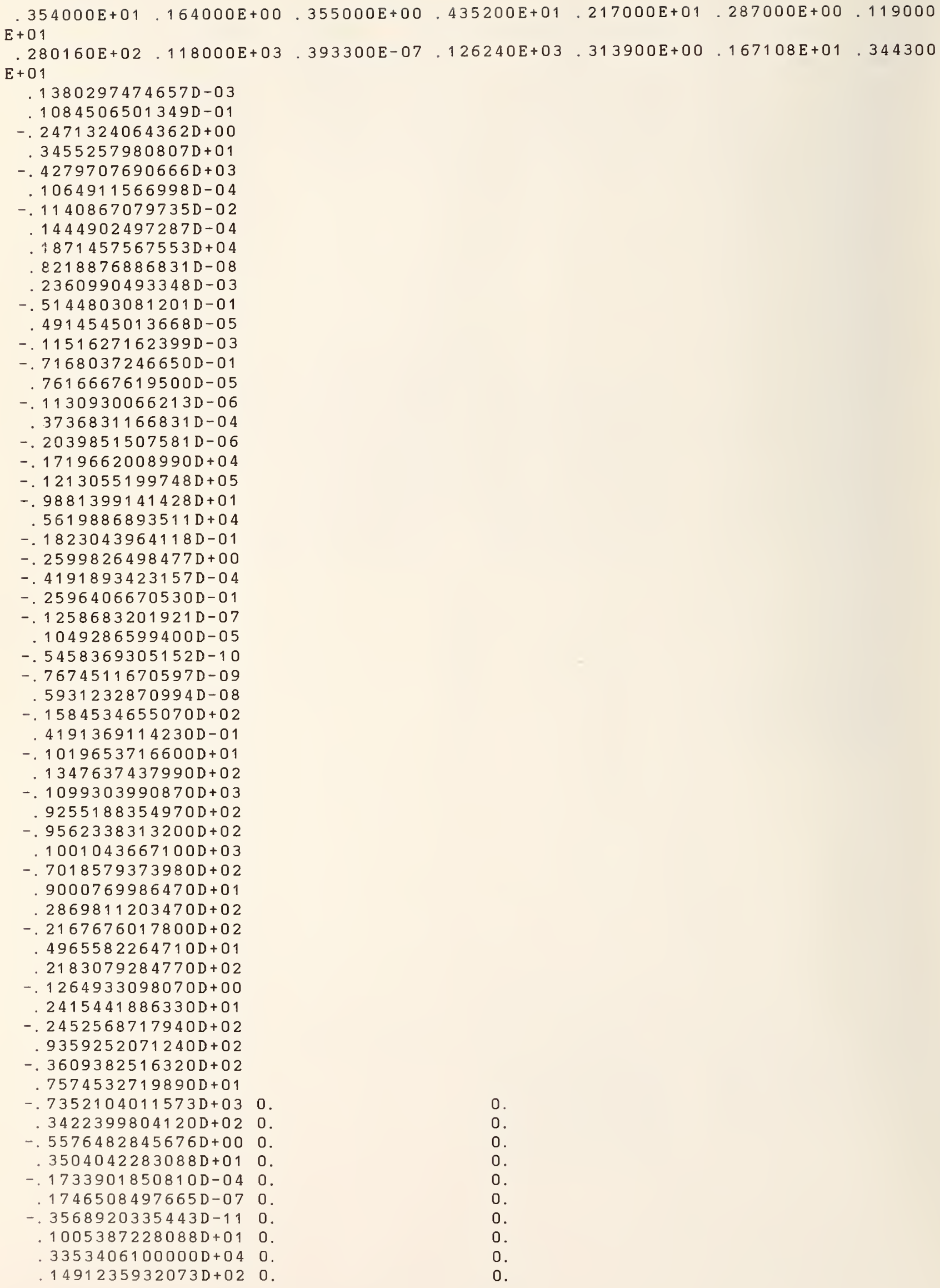


$.2553079069000 \mathrm{D}+020$.

$5111319209400 D+01-.1822424000000 D+05$ $6482667539000 D+00$

-. $1510873091600 \mathrm{D}+00$

0 .

$7402849334200 \mathrm{D}+00$

$1500000000000 \mathrm{D}+01$

$6315000000000 D+02$

$1262600000000 \mathrm{D}+03$

$1246297500000 \mathrm{D}-01$

$-.1212815412900 \mathrm{D}+02$

$5715609213900 \mathrm{D}+00$

$1609461114800 \mathrm{D}+02$

$3695408615800 \mathrm{D}+04$

$-.8088980118000 \mathrm{D}+03$ $6846443564000 \mathrm{D}+02$

-. $2124113591200 D+01$ $3150000000000 \mathrm{D}+00$

-. $1121773962300 \mathrm{D}+00$ $3291231724400 \mathrm{D}-01$ $1400000000000 \mathrm{D}+01$ $1180000000000 \mathrm{D}+03$ $3097717741477 \mathrm{D}+02$

0 .

$00831411-56000000 \mathrm{E}$ $.4399383600000 \mathrm{D}-02$ . $1893209600000 \mathrm{D}-05$

0 . . $1991532737400 \mathrm{D}+05$ -. $9154232449400 \mathrm{D}+04$ . $2325548405900 \mathrm{D}+04$

-. $3630721422800 D+03$ . $3645750681100 \mathrm{D}+02$ -. $2226188081700 \mathrm{D}+01$ . $7805390489500 \mathrm{D}-01$ - $1189402910400 \mathrm{D}-02$ -. $3861329162700 D+02$ 0 .

. $3720174333300 \mathrm{D}+02$ 0 .

-. $3901350907900 \mathrm{D}+02$

-. $3182610948500 \mathrm{D}+02$

0 .

. $1000000000000 \mathrm{D}+01$ $.5387566663700 \mathrm{D}-01$

$6102791110400 \mathrm{D}-02$

. $1200000000000 \mathrm{D}+01$

$.1180000000000 \mathrm{D}+03$ . $2428220857100 \mathrm{D}-01$

0 . $021900.00 \quad 63.15$
$-.1600002810000 D+03$ . $9385755020000 \mathrm{D}-01$ . $1795000000000 \mathrm{D}+01$
0 .

-. $2002957397200 \mathrm{D}+02$

$4976574668400 \mathrm{D}+01$

$.8018895937800 \mathrm{D}+01$

$-.5502271688800 \mathrm{D}+01$

. $1536373896500 \mathrm{D}+01$

-. $2297473725700 \mathrm{D}+00$

. $1936054734600 \mathrm{D}-01$

-. $8567738576800 \mathrm{D}-03$ . $1556467093500 \mathrm{D}-04$
0.

-. $3145017800000 \mathrm{D}-080$.

-. $2859270300000 \mathrm{D}-060$.

-. 4466603400000D-07 0.

02. $\mathrm{COF}$

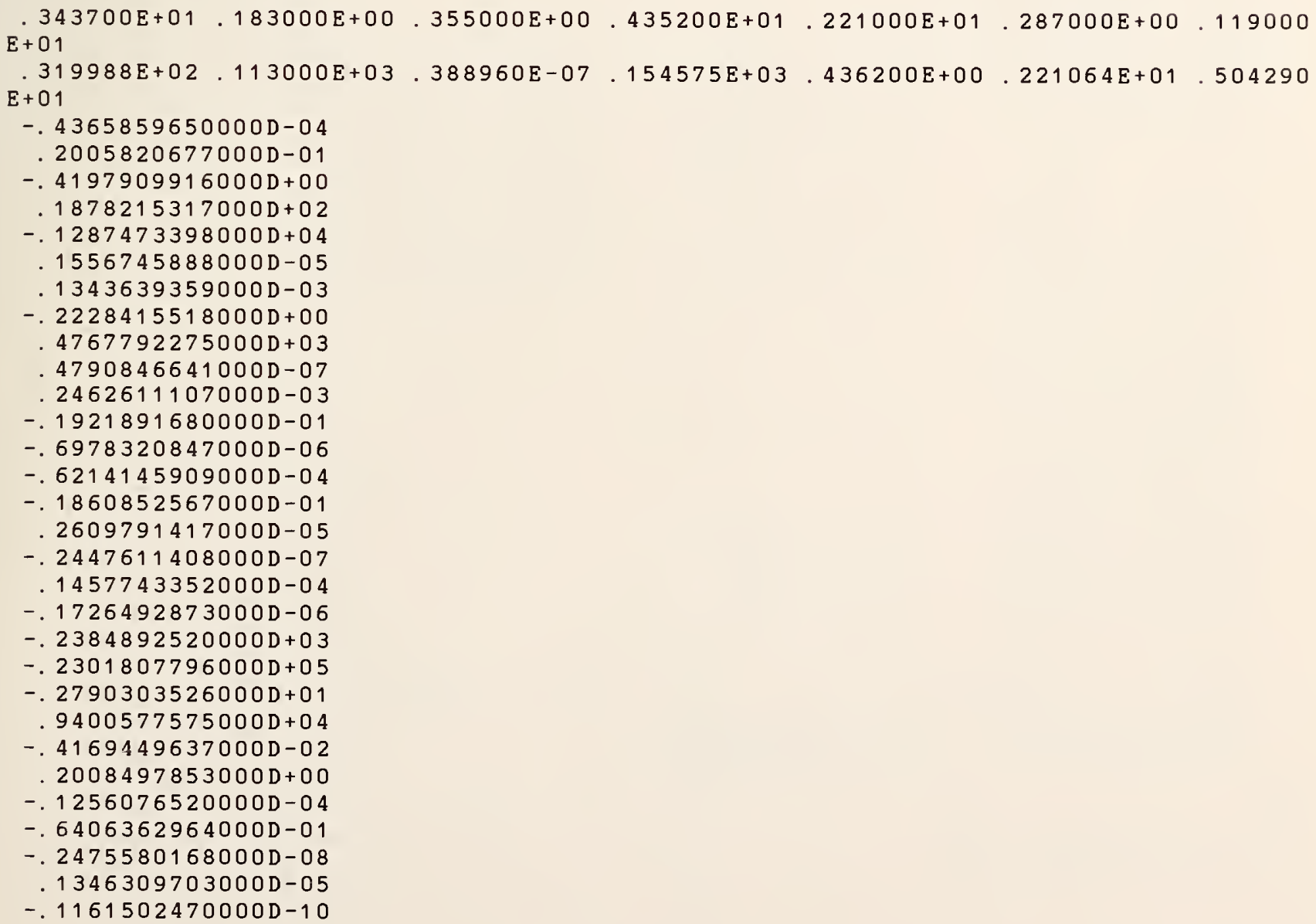


-. $1034699798000 D-08$ . $2365936964000 D-07$ . $5813947530760 \mathrm{D}+02$

-. $4902411961330 \mathrm{D}-01$ . $1683288932520 \mathrm{D}+01$

-. $3251612233980 D+02$ $.5503009898720 D+03$

-. $5109685061150 D+03$ . $3150915590490 D+03$

-. $2325666592580 D+02$

-. $4884254793590 \mathrm{D}+02$

-. $1506242175230 \mathrm{D}+03$ $2804416038510 \mathrm{D}+03$

$-.1766938968610 \mathrm{D}+03$ . $4032477474490 \mathrm{D}+02$ . $2521986883650 \mathrm{D}+01$

-. $1360983164720 \mathrm{D}-01$ $.2823161594030 D+00$

-. $2866459053410 \mathrm{D}+01$

$.6170242122840 \mathrm{D}+01$

-. $8102207954620 \mathrm{D}+00$

-. $2796010689690 \mathrm{D}+00$

-. $4981998537119 \mathrm{D}+040$

$.2302477799952 \mathrm{D}+030$.

-. $3455653235107 \mathrm{D}+010$. . $3521876773671 \mathrm{D}+010$.

-. $4354202160244 \mathrm{D}-040$.

. $1346353450132 \mathrm{D}-070$.

. $1620598259591 \mathrm{D}-100$.

. $1031468515726 \mathrm{D}+010$.

$.2239181050000 D+040$.

$1238529263264 \mathrm{D}+030$.

. $3816718907989 \mathrm{D}+020$.

. $7568956000000 \mathrm{D}+01$

$5004836000000 \mathrm{D}+01$

-. $2137460000000 D+01$

0 .

. $3454481000000 \mathrm{D}+01$

. $1514000000000 D+01$

$.5435900000000 D+02$

$.1545810000000 D+03$

. $1479953000000 D-03$

-. $1445497211000 \mathrm{D}+02$

-. $3142172899400 \mathrm{D}+00$

. $1820116146800 D+02$ $2739042952500 \mathrm{D}+03$

-. $2749895694800 \mathrm{D}+04$ $2434068966700 \mathrm{D}+03$ . $1191150410400 \mathrm{D}+03$

$.4350000000000 D+00$ . $4352651515300 D+00$

-. $2036126387800 \mathrm{D}+00$ $1400000000000 D+01$ $1000000000000 D+03$ . $4081997364372 \mathrm{D}+02$ 0 .

$-.9707637859300 D+04$ $8280125420100 D+04$

-. $2466875880300 \mathrm{D}+04$ . $2132436024300 D+03$ . $3785104952200 \mathrm{D}+02$

-. $1048721609000 \mathrm{D}+02$ $.1113444130400 \mathrm{D}+01$

-. $5367609375700 D-01$

. $1027937964100 \mathrm{D}-02$

-. $2152074113700 D+02$

0 .

. $1679950426100 \mathrm{D}+02$

0 .

-. $2994487872100 \mathrm{D}+04$ . $4735050878800 \mathrm{D}+03$ 0 .

$1000000000000 D+01$ $3060000000000 \mathrm{D}-01$ . $2785000000000 \mathrm{D}-01$ $.1120000000000 D+01$ $.1000000000000 D+03$ . $3318894767078 \mathrm{D}-03$ 0 .

$00831411-.56000000 \mathrm{E}-02 \quad 1500.00 \quad 54.36$ $3960810000000 D-02-.2672268540000 D+03$ $2970000000000 D-06.2276063480000 D+00$

-. $4130000000000 \mathrm{D}-07$

0 .

. $1769000000000 D+01$

0 .

0 .

-. $2140000000000 \mathrm{D}-070$.

0 .

$120.0013 .6300 \quad 5.0430$ 
NF 3. COF

0.

$710190 \mathrm{E}+020$.

$1774353868000 \mathrm{D}-02$

- $5409379418000 \mathrm{D}-01$

$3976634466000 \mathrm{D}+00$

-. $5209476694000 \mathrm{D}+02$

-. $3286322888000 \mathrm{D}+04$

-. $5990517411000 \mathrm{D}-04$

$.9217525601000 \mathrm{D}-01$

-. $4848977075000 \mathrm{D}+02$

-. $4235892691000 \mathrm{D}+06$

-. $9824248063000 \mathrm{D}-06$

$5432235989000 \mathrm{D}-02$

-. $1462388500000 \mathrm{D}+01$

-. $3366180440000 \mathrm{D}-03$ $2801374599000 D-01$ $8435288597000 \mathrm{D}+00$

-. $1324421452000 D-02$ $1875604377000 \mathrm{D}-04$ $2959643991000 \mathrm{D}-01$

$-.7009976870000 \mathrm{D}-03$ $.4365820912000 \mathrm{D}+06$

-. $1111397536000 \mathrm{D}+07$ $.2411866612000 \mathrm{D}+04$ $3179136276000 \mathrm{D}+06$ $6166849090000 \mathrm{D}+01$ $4260854720000 \mathrm{D}+01$ $1090598789000 \mathrm{D}-01$

$-.3340951059000 \mathrm{D}+01$ $8597429644000 \mathrm{D}-05$ . $1240544214000 \mathrm{D}-03$ . $1286224248000 \mathrm{D}-07$

-. $8941104276000 \mathrm{D}-07$ . $3353054595000 \mathrm{D}-05$ . $1312851816360 \mathrm{D}+03$

$-.2269985555360 \mathrm{D}+01$ $3847083894980 \mathrm{D}+02$

-. $3098272682390 \mathrm{D}+03$

-. $6353485266350 \mathrm{D}+03$ $9269790283570 \mathrm{D}+03$ $3714980112590 \mathrm{D}+03$

-. $1822916544700 \mathrm{D}+04$ $1055924038530 \mathrm{D}+04$ $1216738953440 \mathrm{D}+04$

-. $2072089283230 \mathrm{D}+04$ $1134633947100 \mathrm{D}+04$

-. $2266421371400 \mathrm{D}+03$ $1050872951730 \mathrm{D}+01$

-. $1316864742460 \mathrm{D}-02$ $3901413319000 D-01$

-. $5877965979750 \mathrm{D}+00$ $4009005210170 \mathrm{D}+01$

$-.2033853869770 \mathrm{D}+01$ $4004343644240 \mathrm{D}+00$ $7427518245951 \mathrm{D}+060$.

-. $4389825372134 \mathrm{D}+050$. . $1012629224351 \mathrm{D}+040$.

$-.7140693612211 \mathrm{D}+010$. $.5481339146452 \mathrm{D}-010$.

-. $7677196006769 \mathrm{D}-040$. . $4203630864340 \mathrm{D}-070$.

-. $6328752997967 \mathrm{D}+000$. . $3000000000000 \mathrm{D}+040$.

-. $3493688822979 \mathrm{D}+050$. $5145589888845 \mathrm{D}+030$.
0 .

0 .

0 .

0 .

0 .

0 . $.234000 E+03.792000 E+01.171240 E+010$.
0.

0.

0.

0.

0.

0.

0 .

0.

0 .

0 .

0 . 


\begin{tabular}{|c|c|c|c|c|}
\hline & & & & \\
\hline $9654850231200 \mathrm{D}+01$ & 0. & 0. & & \\
\hline$-.2872773281500 \mathrm{D}+01$ & 0 . & 0 . & & \\
\hline$-.1379770070000 D+01$ & 0. & 0 . & & \\
\hline $.7311244167300 D+01$ & 0. & 0. & & \\
\hline $.1500000000000 D+01$ & 0. & 0. & & \\
\hline $.6636000000000 D+02$ & 0 . & 0 . & & \\
\hline $.2340000000000 D+03$ & 0 . & 0 . & & \\
\hline $.1853782700000 \mathrm{D}-06$ & 0 . & 0 . & & \\
\hline 0. & 0. & & & \\
\hline 0 . & 0 . & & & \\
\hline 0 . & 0. & & & \\
\hline 0. & 0 . & & & \\
\hline 0. & 0 . & & & \\
\hline 0. & 0 . & & & \\
\hline 0. & 0. & & & \\
\hline 0 . & 0. & & & \\
\hline 0 . & 0 . & & & \\
\hline 0 . & 0. & & & \\
\hline 0 . & 0 . & & & \\
\hline 0. & 0 & & & \\
\hline $.2632000000000 \mathrm{D}+02$ & $.3361200000000 \mathrm{D}-08$ & & & \\
\hline 0. & 0 & 0 . & & \\
\hline $.00831441-.56000000 E$ & $\begin{array}{lll}E-02 & 500.00 \quad 66.36\end{array}$ & 50.00 & 7.9200 & 4. 4607 \\
\hline 0. & $-.1909399710000 \mathrm{D}+03$ & & & \\
\hline 0 . & $.8137501940000 \mathrm{D}-01$ & & & \\
\hline 0. & $.1850000000000 D+01$ & & & \\
\hline 0. & 0. & & & \\
\hline 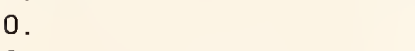 & 0. & & & \\
\hline o. & 0. & & & \\
\hline
\end{tabular}

METH. COF

0.

0 .

$.9898937956000 \mathrm{D}-05$

. $2199608275000 \mathrm{D}-01$

-. $5322788000000 \mathrm{D}+00$

$.2021657962000 \mathrm{D}+02$

-. $2234398926000 \mathrm{D}+04$

. $1067940280000 \mathrm{D}-04$

$.1457922469000 \mathrm{D}-03$

-. $9265816666000 \mathrm{D}+00$

$.2915364732000 \mathrm{D}+03$

$.2313546209000 \mathrm{D}-06$

. $1387214274000 \mathrm{D}-03$

. $4780467451000 \mathrm{D}-02$

$1176103833000 \mathrm{D}-04$

-. $1982096730000 \mathrm{D}-03$

-. $2512887756000 \mathrm{D}-01$

$.9748899826000 \mathrm{D}-05$

-. $1202192137000 \mathrm{D}-06$

. $4128353939000 \mathrm{D}-04$

-. $7215842918000 \mathrm{D}-06$

$.5081738255000 D+03$

-. $9198903192000 \mathrm{D}+05$

-. $2732264677000 \mathrm{D}+01$

$7499024351000 \mathrm{D}+05$

$1114060908000 \mathrm{D}-02$

$1083955159000 D+01$

-. $4490960312000 \mathrm{D}-04$

-. $1380337847000 D+01$

-. $2371902232000 D-07$

$.3761652197000 \mathrm{D}-04$

-. $2375166954000 \mathrm{D}-09$

-. $1237640790000 \mathrm{D}-07$ $.6766926453000 \mathrm{D}-\mathrm{nF}$
0.

0 .

0.

0 .
0.

0 .
0.

0 .
0.

0. 
. $4436658614060 D+00$ $6172912655850 D+00$

$-.1714092038860 D+01$ $2664891756670 D+01$

$-.2006798722650 \mathrm{D}+01$ $5054513976350 D+00$

-. $1255990487150 \mathrm{D}+01$ . $1374552965910 \mathrm{D}-02$

-. $6643534109710 D-01$ $.1277137136070 D+01$

-. $8438642963590 D+01$ $3101115288610 D+01$

-. $5753632727380 D+00$

0 .

0 .

0 .

0 .

0 .

0.

0 .

-. $1804475050700 \mathrm{D}+070$. $.7742666639300 \mathrm{D}+050$.

-. $1324165875400 \mathrm{D}+040$. $.1543814959500 \mathrm{D}+020$.

-. $5147900525700 \mathrm{D}-010$. $1080917219600 \mathrm{D}-030$.

-. $5550178343700 \mathrm{D}-070$.

-. $6749005617100 D+010$. $.3000000000000 D+040$. $4869146318103 \mathrm{D}+050$.

-. $4861658943347 \mathrm{D}+030$. $4779399530000 \mathrm{D}+010$. $1757393941000 \mathrm{D}+010$.

$-.5665073910000 D+000$. 0 .

$1326326855000 \mathrm{D}+01$

0 . $1500000000000 \mathrm{D}+010$. . $9068000000000 \mathrm{D}+020$. . $1905550000000 D+030$. $1174300000000 \mathrm{D}-010$.

-. $1620427429000 \mathrm{D}+02$ $4270589027000 \mathrm{D}+03$ . $1402596278000 \mathrm{D}+02$

-. $3916837745000 D+04$

-. $3477099090000 D-01$ . $2136542674000 D+02$ . $1436802482000 \mathrm{D}+04$

0 . $1696985927100 \mathrm{D}+000$.

$-.1333723450800 D-010$.

$.1400000000000 D+010$.

. $1680000000000 D+030$.

$.2814700000000 \mathrm{D}+02 \quad .1567900000000 \mathrm{D}-01$ 0 . 0 .

$2325800819000 D-02$ $.2477927999000 D+00$ . $3880593713000 D+02$ -. $1579519146000 \mathrm{D}-06$ . $3717991328000 \mathrm{D}-02$ $-.9616989434000 D+00$ -. $3017352774000 D-01$ $4298153386000 D+00$

$$
\text { . }
$$

$.00831434-.97066175 \mathrm{E}-02 \quad 600.00 \quad 90.68$

$.6570101800000 D-02-.1909269420000 D+03$

$.6383501300000 \mathrm{D}-05.4565597600000 \mathrm{D}-01$

$-.1871872800000 \mathrm{D}-06.1850000000000 \mathrm{D}+01$

$-.5313482000000 D-040$.

$-.9471173500000 \mathrm{D}-060$.

0 .

0 .

0 .

0 .

0

0 .

0 .

0 .

0 .

0 .

0 .

0 .

0 .

0 .

. $1346953698000 \mathrm{D}+01$

-. $3254677753000 D+00$

0 .

0 .

C.

0 .

0 .

0 .

0 .

$.287000 \mathrm{E}+00.119000 \mathrm{E}+01.355000 \mathrm{E}+00.435200 \mathrm{E}+01.138054 \mathrm{E}-22.217000 \mathrm{E}+01$ $164000 \mathrm{E}+00.651271 \mathrm{E}-09.374237 \mathrm{E}+02.316714 \mathrm{E}+01.780350 \mathrm{E}+00.601030 \mathrm{E}+00$ $160420 \mathrm{E}+02.168000 \mathrm{E}+03.368000 \mathrm{E}+01.520000 \mathrm{E}+00$ 
C $2 \mathrm{H} 4$. $\mathrm{COF}$

0 . $280540 E+020$

-. $2146684366683 \mathrm{D}-02$

. $1791433722534 \mathrm{D}+00$

-. $3675315603930 \mathrm{D}+01$ $3707178934669 \mathrm{D}+03$

-. $3198282566709 \mathrm{D}+05$ . $5809379774732 \mathrm{D}-04$

-. $7895570824899 \mathrm{D}-01$

$.1148620375835 \mathrm{D}+02$ $.2713774629193 \mathrm{D}+05$

-. $8647124319107 \mathrm{D}-05$ $.1617727266385 \mathrm{D}-01$

-. $2731527496271 \mathrm{D}+01$

-. $2672283641459 \mathrm{D}-03$

-. $4752381331990 \mathrm{D}-02$

-. $6255637346217 \mathrm{D}+01$ $.4576234964434 \mathrm{D}-03$

-. $7534839269320 \mathrm{D}-05$

$.1638171982209 \mathrm{D}-01$

-. $3563090740740 \mathrm{D}-03$

-. $1833000783170 \mathrm{D}+05$

-. $1805074209985 \mathrm{D}+07$

-. $4794587918874 \mathrm{D}+03$

. $3531948274957 \mathrm{D}+07$

-. $2562571039155 \mathrm{D}+01$ . $1044308253292 D+03$

-. $1695303363659 \mathrm{D}-01$

-. $1710334224958 \mathrm{D}+03$

-. $2054114462372 \mathrm{D}-04$ $.6727558766661 \mathrm{D}-02$

-. $1557168403328 \mathrm{D}-06$

-. 1229814736077 D-04

$.4234325938573 \mathrm{D}-04$

-. $6096215155940 \mathrm{D}+02$ . $2031853127020 \mathrm{D}-01$

-. $9254412658130 \mathrm{D}+00$ $2436307958880 D+02$

$-.8547456228880 \mathrm{D}+03$ $1239278681830 \mathrm{D}+04$

-. $1427107117890 \mathrm{D}+04$ $8373586704050 D+03$

$4322036965520 \mathrm{D}+03$

-. $1379175411610 \mathrm{D}+04$ $1268586001240 D+04$

$-.5715523217130 \mathrm{D}+03$ $1060122343600 \mathrm{D}+03$

-. $4790470601830 \mathrm{D}+01$ $1513813452830 D-01$

-. $4034560794450 \mathrm{D}+00$ $5086839202250 \mathrm{D}+01$

-. $2467119979870 \mathrm{D}+02$ $9800309152470 \mathrm{D}+01$

-. $2168465161220 \mathrm{D}+01$ $5603615762000 \mathrm{D}+060$.

-. $2141069802000 \mathrm{D}+050$. $2532008897000 \mathrm{D}+030$. $3554495281000 \mathrm{D}+010$.

-. $9951927478000 \mathrm{D}-020$. 5108931070000D-040.

-. $1928667482000 \mathrm{D}-070$.

-. $2061703241000 \mathrm{D}+020$. $3000000000000 D+040$.

-.1054840584320D+050. $6447430891887 \mathrm{D}+020$.
0.0 . 0

0 .

0 .

0 .

0 . 0. $.167108 \mathrm{E}+010$.
0 .

0 .

0 .

0 .

0 .

0 .

0 .

0 .

0 .

0 .

0 . 


\begin{tabular}{|c|c|c|c|c|}
\hline & & & & \\
\hline $\begin{array}{l}8209579800000 D+01 \\
4315424145000 D+01\end{array}$ & $\begin{array}{l}0 . \\
0 .\end{array}$ & $\begin{array}{l}0 . \\
0 .\end{array}$ & & \\
\hline -. $1692585975000 D+01$ & 0. & 0. & & \\
\hline -. $1976495575000 \mathrm{D}+00$ & 0. & 0. & & \\
\hline $.3446501098000 \mathrm{D}+01$ & 0. & 0 . & & \\
\hline $1500000000000 D+01$ & 0. & 0 . & & \\
\hline $.1039860000000 D+03$ & 0. & 0. & & \\
\hline $.2823428000000 \mathrm{D}+03$ & 0 . & 0 . & & \\
\hline $.1212951400000 D-03$ & 0. & 0. & & \\
\hline 0. & 0. & & & \\
\hline 0 . & 0. & & & \\
\hline 0. & 0. & & & \\
\hline 0. & 0. & & & \\
\hline 0. & 0. & & & \\
\hline 0. & 0. & & & \\
\hline 0. & 0. & & & \\
\hline 0. & 0. & & & \\
\hline 0. & 0. & & & \\
\hline 0. & 0. & & & \\
\hline 0. & 0. & & & \\
\hline & 0. & & & \\
\hline $.2334296694034 \mathrm{D}+02$ & $.1425455127094 \mathrm{D}-03$ & & & \\
\hline $0.00831434-.17200000 \mathrm{E}$ & $\begin{array}{lll}0 . & \\
-01 & 400.00 & 103.99\end{array}$ & 0.40 .00 & 7.6500 & 5.0404 \\
\hline 0. & $-.3579238750000 \mathrm{D}+03$ & & & \\
\hline 0. & $.2453321430000 \mathrm{D}-01$ & & & \\
\hline 0. & $.2064500000000 D+01$ & & & \\
\hline 0. & 0 & & & \\
\hline 0. & 0. & & & \\
\hline 0. & 0. & & & \\
\hline
\end{tabular}

C2 H6. COF

0.

0.

-. $3204748852000 \mathrm{D}-03$

$.6529792241000 \mathrm{D}-01$

-. $1669704591000 \mathrm{D}+01$

$.1147983381000 \mathrm{D}+03$

-. $1854721998000 \mathrm{D}+05$ $.4994149431000 \mathrm{D}-04$

-. $4858871291000 \mathrm{D}-01$ $.1225345776000 \mathrm{D}+02$ $.8622615988000 \mathrm{D}+04$

-. $1081290283000 \mathrm{D}-05$ $.6279096996000 \mathrm{D}-02$

$-.1716912675000 \mathrm{D}+01$

-. $1640779401000 \mathrm{D}-04$

$-.4356516111000 \mathrm{D}-02$

$-.1966649699000 \mathrm{D}+01$ $4026724698000 \mathrm{D}-03$

-. $6498241861000 \mathrm{D}-05$ $5111594139000 \mathrm{D}-02$

-. $1113010349000 \mathrm{D}-03$

-. $7157747547000 \mathrm{D}+03$

-. $1848571024000 \mathrm{D}+07$

-. $2137365569000 \mathrm{D}+03$ $.6275079986000 \mathrm{D}+07$

-. $9974911056000 D+00$ $1129115014000 \mathrm{D}+03$

-. $1026469558000 \mathrm{D}-01$

-. $5660525915000 \mathrm{D}+03$

-. $4209846430000 \mathrm{D}-04$ $2374523553000 \mathrm{D}-01$

-. $1289637823000 \mathrm{D}-06$

-. $5423801068000 \mathrm{D}-04$ $2239717230000 \mathrm{D}-02$ . $3557074081980 \mathrm{D}+00$
0.

0 .

0.

0 .
0.

0 .
0.

0.
0.

0 . 
. $1099839659870 \mathrm{D}+00$

. $1371418636620 \mathrm{D}+00$

-. $9801225235070 \mathrm{D}+00$

. $1338489436060 D+01$

$-.8076017312800 \mathrm{D}+00$

-. $1884527431980 \mathrm{D}+01$

$4486631930080 \mathrm{D}-02$

$-.1248903769190 \mathrm{D}+00$

. $1797380770930 \mathrm{D}+01$

$-.1375304922580 \mathrm{D}+02$

. $6941377045750 \mathrm{D}+01$

-. $1932968312710 \mathrm{D}+01$

0 .

0 .

0 .

0 .

0 .

0 .

-. $6934140690900 \mathrm{D}+06$

. $3153483413500 \mathrm{D}+050$.

0.

-. $6103375287000 \mathrm{D}+030$

0.

$.9950792245900 \mathrm{D}+010$.

0 .

-. $2865787794800 \mathrm{D}-010$.

. 9092289782100D-04 0.

0 .

-. 5275010991500D-07 0.

0 .

0 .

-. $1424359341100 \mathrm{D}+020$.

. $3000000000000 \mathrm{D}+040$.

0 .

$2176008367499 \mathrm{D}+050$.

0 .

0 .

-. $2165173422429 D+030$.

0 .

$8900377023000 \mathrm{D}+010$.

0 .

$.1155950893100 D+020$.

-. 4009244950000D+010.

0 .

0 .

-. $1175263997000 D+010$.

0 .

$.7918066027000 \mathrm{D}+010$.

0 .

$.1600000000000 \mathrm{D}+010$.

0 .

$.9034800000000 \mathrm{D}+020$.

0 .

. $3053300000000 \mathrm{D}+030$.

0 .

0 .

$1130842618000 \mathrm{D}-050$.

0 .

0 .

0 .

0 .

0 .

0 .

0 .

$0 . \quad 0$

0.

$0 . \quad 0$

0 .

$.2102436247000 \mathrm{D}+010$

$-.1065920192000 \mathrm{D}+010$.

$.1400000000000 D+010$.

. $3053300000000 \mathrm{D}+030$.

$.2168000000000 \mathrm{D}+02.1515400000000 \mathrm{D}-05$

0 .

0

$.00831434-.21157025 \mathrm{E}-01 \quad 600.00 \quad 90.35$ $.1114210000000 \mathrm{D}-01-.2559650000000 \mathrm{D}+03$ $.2066220000000 \mathrm{D}-04.1400342000000 \mathrm{D}-01$

-. $1359820000000 \mathrm{D}-05 \quad .2179000000000 \mathrm{D}+01$

. $6304320000000 \mathrm{D}-040$.

-. $1456830000000 \mathrm{D}-060$.

0 .

0 .
. $168000 \mathrm{E}+00.742399 \mathrm{E}-090$.

$300701 E+02.240000 E+03.440110 E+01$
0 .
$435200 \mathrm{E}+01$
0 .

0 .
70.00
6. 8750
4. 8714 $350000 E+00$ 
C3H8. COF

0.

0.

-. $2804337729000 \mathrm{D}-03$ $1180666107000 \mathrm{D}+00$

-. $3756325860000 \mathrm{D}+01$ $.5624374521000 \mathrm{D}+03$

-. $9354759605000 \mathrm{D}+05$

-. $4557405505000 \mathrm{D}-04$ $.1530044332000 \mathrm{D}+00$

-. $1078107476000 \mathrm{D}+03$ $2218072099000 \mathrm{D}+05$ $6629473971000 \mathrm{D}-05$

-. $6199354447000 D-02$ $.6754207966000 \mathrm{D}+01$ $6472837570000 \mathrm{D}-03$

-. $6804325262000 \mathrm{D}-01$

-. $9726162355000 D+01$ $5097956459000 \mathrm{D}-02$

-. $1004655900000 \mathrm{D}-03$ $.4363693352000 \mathrm{D}-01$

-. $1249351947000 \mathrm{D}-02$ $2644755879000 \mathrm{D}+05$

$-.7944237270000 \mathrm{D}+07$

-. $7299920845000 \mathrm{D}+03$ . $5381095003000 \mathrm{D}+08$ $3450217377000 \mathrm{D}+01$ $9936666689000 D+03$

$-.2166699036000 \mathrm{D}+00$

-. $1612103424000 \mathrm{D}+05$

-. $3633126990000 \mathrm{D}-03$ $1108612343000 \mathrm{D}+01$

$-.1330932838000 \mathrm{D}-04$

-. $3157701101000 \mathrm{D}-02$ $1423083811000 \mathrm{D}+00$ $2776096607720 \mathrm{D}+00$ $9963162115260 \mathrm{D}-01$

-. $9351030114790 \mathrm{D}-01$

-. $3931811933810 D+00$ $7803933323340 \mathrm{D}+00$

-. $5946726552360 \mathrm{D}+00$

-. $1703537178580 \mathrm{D}+02$ $8507185809450 \mathrm{D}-01$

-. $1698995082710 \mathrm{D}+01$ $.1842068338990 \mathrm{D}+02$

$-.8153344355910 \mathrm{D}+02$ $3306123402780 \mathrm{D}+02$

-. $7376365110310 \mathrm{D}+01$

0 .

0 .

0 .

0 .

0 .

0 .

0 .

$3125245009900 \mathrm{D}+070$.

$-.1141525363800 \mathrm{D}+060$. . $1497165072000 \mathrm{D}+040$.

$-.5404120433800 \mathrm{D}+010$. . 3921545289700D-010.

-. $2173891392600 \mathrm{D}-040$. $.4827454130300 \mathrm{D}-080$. . $3190701634900 \mathrm{D}+010$. . $1500000000000 \mathrm{D}+040$.

$-.5905992207939 \mathrm{D}+050$. $.4723601648251 \mathrm{D}+030$. $\begin{array}{ll}0 . & 0 . \\ 0 . & 0 .\end{array}$

0 .

0.

0 .

0.

0 .

0 .
0.

0 .

0 .

0.

0.

0 .

0 .

0 .

0 .

0 .

0 . 


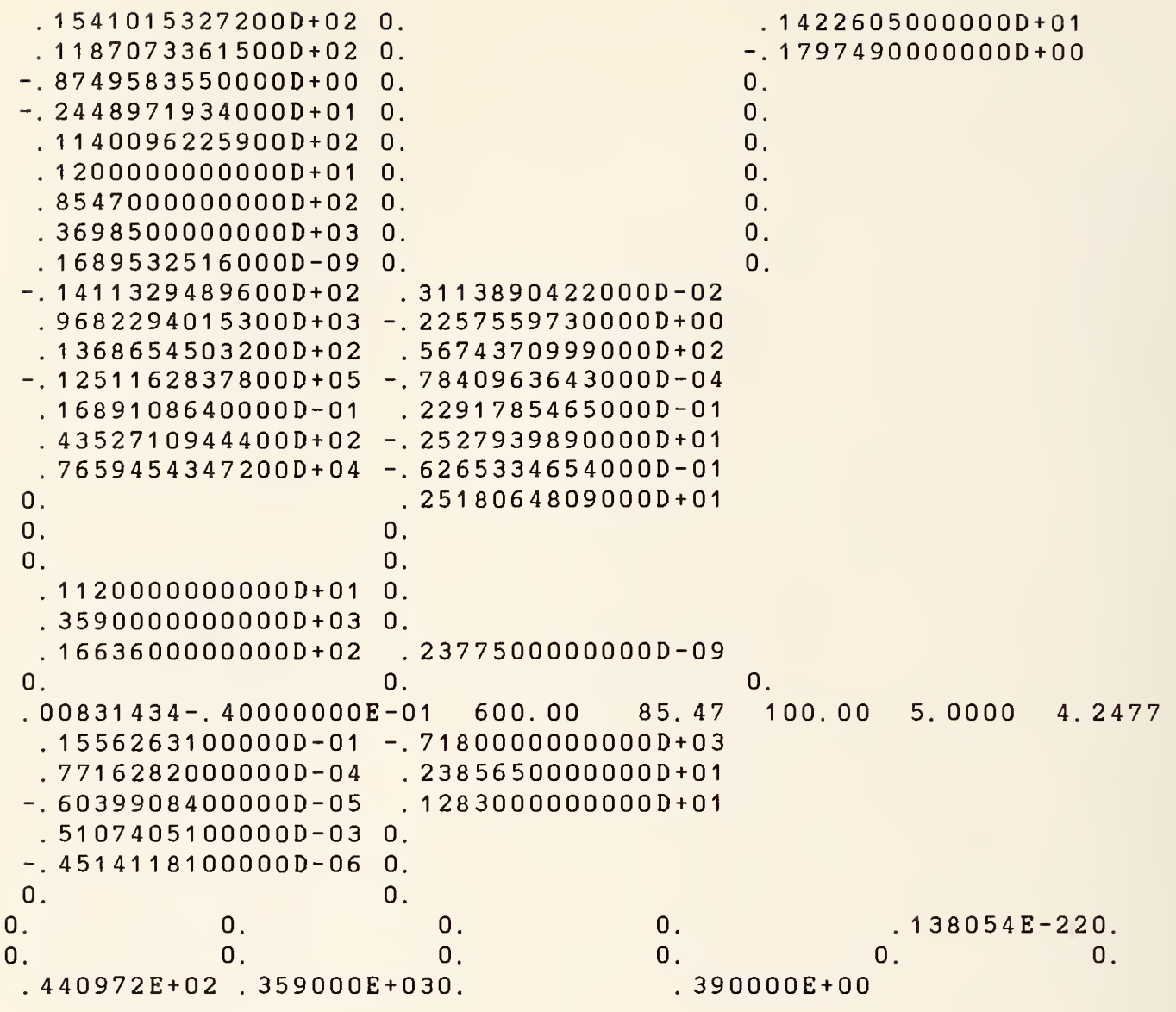

I SOB. COF

$\begin{array}{ll}0 . & 0 \\ 0 . & 0\end{array}$

. $1307325972000 \mathrm{D}-02$ $3927802742000 D-01$

-. $3185427394000 D+01$ . $7608825192000 D+03$

-. $1753919859000 D+06$

-. $2090019755000 D-03$ $.8959557971000 D+00$

-. $6816710130000 D+03$

-. $1111271045000 D+06$

. $3248737572000 D-04$

-. $1046526456000 D+00$ . $6536598969000 D+02$ . $3726503734000 D-02$ $.8553649395000 D+00$ . $2109987236000 D+03$

-. $1401267363000 \mathrm{D}+00$ $.5213089327000 \mathrm{D}-02$

-. $1925026382000 D+01$ . $7640067895000 \mathrm{D}-01$ . $3425854273000 \mathrm{D}+06$

-. $3373475924000 \mathrm{D}+08$

. $1180683444000 D+05$ . $1529683738000 D+09$ . $3323837416000 D+03$ . $6423169487000 \mathrm{D}+04$ $3891706042000 D+01$

$-.1494755736000 \mathrm{D}+06$

-. $1720240173000 D-02$

. $2894195375000 \mathrm{D}+02$ . $2005086329000 \mathrm{D}-03$
$0 . \quad 0$.

0 .

0 .
0.

0 .
0 .

0 .
0.

0 . 
-. $4448393005000 \mathrm{D}-01$ $8028488415000 \mathrm{D}+01$ $3432512286730 \mathrm{D}+00$ $4828577762610 \mathrm{D}-01$ $5484324610530 \mathrm{D}+00$

-. $1884263998340 D+01$ $2028124065780 \mathrm{D}+01$

-. $9734823703320 \mathrm{D}+00$

-. $9587205839600 \mathrm{D}+01$ $3912955870750 D-01$

-. $8482763798650 \mathrm{D}+00$ $9883018121350 \mathrm{D}+01$

-. $4930250474110 \mathrm{D}+02$ $2097730743050 D+02$

-. $4921886633430 \mathrm{D}+01$

0 .

0 .

0 .

0 .

0 .

0 .

0 .

$1702791900600 \mathrm{D}+080$.

-. $4726972473700 \mathrm{D}+060$. $4730140658100 D+040$.

-. $1723172327800 \mathrm{D}+020$.

$.5849134429100 \mathrm{D}-010$.

$.8944035188600 \mathrm{D}-050$.

-. $1827459919700 \mathrm{D}-070$.

-. $1928302196200 \mathrm{D}+020$. . $3000000000000 D+040$.

-. $1977633864428 \mathrm{D}+060$. $1076385671262 \mathrm{D}+040$.

. $1264889651000 \mathrm{D}+020$.

. $1074641516000 \mathrm{D}+020$.

-. $2191129109000 \mathrm{D}+010$.

-. $2155822575000 D+010$.

$.8832766439000 D+010$.

. $1400000000000 D+010$.

$.1135500000000 D+030$. . $4078500000000 D+030$. $.1948100000000 \mathrm{D}-070$.

-. $2055498053000 D+02$ . $1357076181000 \mathrm{D}+04$

. $4307008989000 \mathrm{D}-02$ . $1893774336000 \mathrm{D}+02$

-. $1509010974000 D+01$ . $4693712392000 \mathrm{D}+03$

$-.1822277344000 D+05$

$-.3554280979000 D-03$

-. $4599387773000 \mathrm{D}-02$ . $6305247065000 \mathrm{D}+02$

. $1841552874000 \mathrm{D}+00$

-. $3892338766000 \mathrm{D}+02$

$1282253921000 \mathrm{D}+05$

- $9354624917000 \mathrm{D}-01$ $7114330590000 \mathrm{D}+01$

$.1687838652000 \mathrm{D}+01$

0 .

$1400000000000 D+010$ $4078500000000 \mathrm{D}+030$. . $1275500000000 \mathrm{D}+02$

0 . 0 .

$2063400000000 D-07$

$00831434-.67115896 \mathrm{E}-01 \quad 600.00 \quad 113.55$ $1986702600000 \mathrm{D}-01-.4300000000000 \mathrm{D}+02$

0 .

0 .

0 .

0 .

0 .

0 .

0 .

0 .

$1449797353000 D+01$

-. $1685643887000 \mathrm{D}+00$

0 .

0 .

0 .

0 .

0 .

0 .

0 .

$\begin{array}{lll}35.00 & 3.8600 & 3.6400\end{array}$ $1760005300000 D-03.1373820000000 D-10$

$-.1526737200000 \mathrm{D}-04.6080000000000 \mathrm{D}+01$ $.9947290400000 \mathrm{D}-030$.

-. 5637502400000D-06 0.

0 .

0 .

$.287000 E+00.119000 E+01.355000 E+00.435200 E+01.138054 E-22.207000 E+01$ $140000 E+00.910218 E-09.347138 E-02.101207 E+02.466392 E+00.100344 E+01$ $581243 E+02.418000 E+03.509217 E+01.250000 E+00$ 
NORB. COF

0 .

0 .

$1537401046030 \mathrm{D}-02$

-. $1609800346110 \mathrm{D}-01$

-. $9797824590100 \mathrm{D}+00$ $4996606745040 \mathrm{D}+02$

-. $1021156076870 \mathrm{D}+06$

. $2360321477560 \mathrm{D}-03$

-. $1374757570930 \mathrm{D}+00$

-. $9070387338650 \mathrm{D}+02$

. $3854217482130 \mathrm{D}+05$

-. $3494537107000 \mathrm{D}-05$

$.1573611227140 \mathrm{D}-0.1$

$.1023014740680 D+02$

. $1823357373310 \mathrm{D}-02$

-. $4041143077870 \mathrm{D}+00$

. $1879798557830 \mathrm{D}+00$

. $3620887950400 D-01$

-. $7387622482660 \mathrm{D}-03$

-. $2186185905630 \mathrm{D}+00$ . $1188027290270 D-01$ $.7068541987130 \mathrm{D}+05$

-. $2194698857960 \mathrm{D}+08$

-. $1824543612680 \mathrm{D}+04$ . $2067903772770 D+09$

. $1117575501450 D+02$ . $5587799259860 \mathrm{D}+04$

- $1595790540260 D+01$

-. $1480342146220 \mathrm{D}+06$

-. $2452063282010 \mathrm{D}-01$ $2183052593090 \mathrm{D}+02$

-. $9239906273380 \mathrm{D}-05$

-. $2052677766390 D+00$ $3876390448200 D+01$

$4041621499070 D+00$ . $9607640867320 \mathrm{D}-01$ $3404715226650 \mathrm{D}+00$

-. $1729592472230 D+01$ $.2214509591330 D+01$

- . $1195654636800 \mathrm{D}+01$

-. $5007387533220 D+01$ . $9593278394870 \mathrm{D}-02$

-. $2995224440540 D+00$ $.4457489568490 \mathrm{D}+01$

-. $3041854550990 D+02$

. $1424771122650 \mathrm{D}+02$

-. $3608627293560 \mathrm{D}+01$

0 .

0 .

0.

0 .

0 .

0 .

0 .

. $3880231019400 \mathrm{D}+060$.

-. $1544429689000 \mathrm{D}+060$. $.2845508223900 D+040$.

-. $1349151137600 \mathrm{D}+020$. $.6614259535300 \mathrm{D}-010$.

-. $2430796502800 \mathrm{D}-040$. . $1504424842900 \mathrm{D}-090$.

-.8393342346700D+010. . $3000000000000 D+040$.

-. $1090160645491 D+060$. $.8662371372633 \mathrm{D}+030$.
0 .

0 .

0 .

0 .

0 .

0 . 
-. $7645674906000 \mathrm{D}+010$. $2457845942700 \mathrm{D}+020$.

$-.1096617182000 \mathrm{D}+010$.

-. $2890833730000 \mathrm{D}+000$. $.2516192927700 \mathrm{D}+020$. $1030000000000 D+010$. $.1348600000000 D+030$. $4251600000000 \mathrm{D}+030$. $6735758052000 \mathrm{D}-060$.

-. $2724386845000 \mathrm{D}+02$ $.8012766611000 \mathrm{D}+03$ $.2503978646000 \mathrm{D}+02$

$.4024170074000 D-02$ $1561435847000 D+01$ $-.6004381127000 D+03$

-.1309704275000D+05-.7547260841000D-03

$-.8313305258000 D-01-.2069676662000 D-01$ $.6636975027000 \mathrm{D}+02.9382534978000 \mathrm{D}+02$ $.9849317662000 \mathrm{D}+04-.1711371457000 \mathrm{D}+00$

0 . $.1630521851000 \mathrm{D}+010$

0 . $1400000000000 \mathrm{D}+010$ $4251600000000 D+030$. $1265000000000 D+02.6007200000000 D-06$

0 . 0 . $3647724935000 D+02$

0 .

$00831434-.65077051 \mathrm{E}-01 \quad 500.00 \quad 134.86$ $.2069763100000 D-01-.3634000000000 D+03$ $.6734573100000 D-04.7134170000000 D-02$

$-.6611178500000 \mathrm{D}-05 \quad .2210000000000 \mathrm{D}+01$

-. $1115193300000 \mathrm{D}-030$.

-. $1202982000000 D-050$.

0 . 0 .

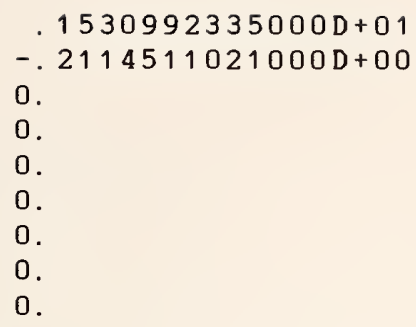

$.287000 \mathrm{E}+00.119000 \mathrm{E}+01.355000 \mathrm{E}+00.435200 \mathrm{E}+01.138054 \mathrm{E}-22.207000 \mathrm{E}+01$ $.140000 \mathrm{E}+00.910218 \mathrm{E}-09.769608 \mathrm{E}-03.132533 \mathrm{E}+02.485554 \mathrm{E}+00.101021 \mathrm{E}+01$ $581243 E+02.440000 E+03.503103 E+01.320000 E+00$ 


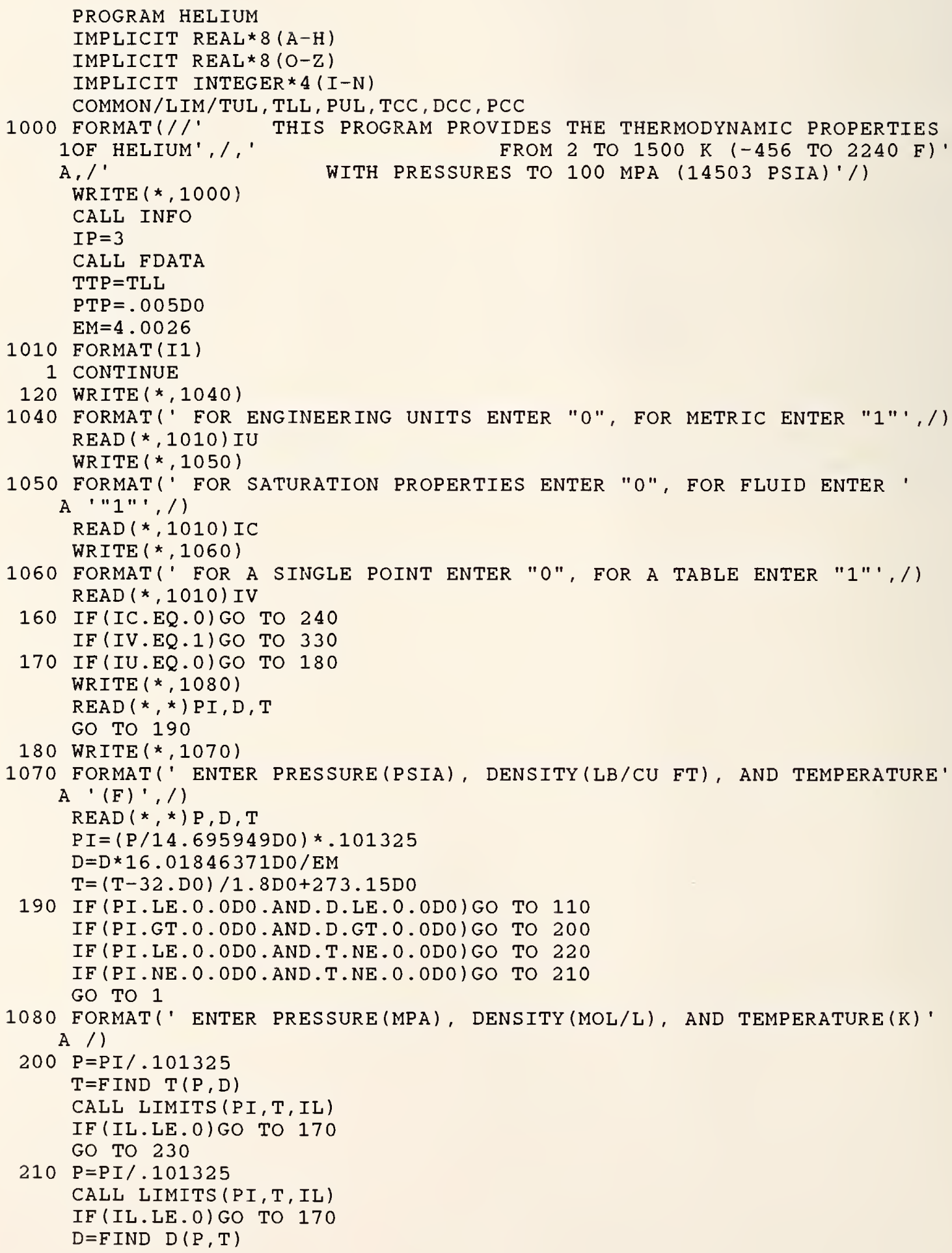


GO TO 230

$220 P=F I N D \quad P(D, T)$

$P I=P^{*} .101325$

CALL LIMITS (PI, T, IL)

IF (IL.LE.O) GO TO 170

230 CALL REPRO (PI, D , T , IU, IV , IC, IP, TF , TDEL )

GO TO 170

240 WRITE $(*, 1090)$

1090 FORMAT(" FOR SATURATED LIQUID ENTER "0", FOR VAPOR ENTER "1",,/)

READ (*,1010) IP

IF (IV.EQ.1) GO TO 330

WRITE $(*, 1095)$

1095 FORMAT(" TO ENTER WITH TEMPERATURE ENTER "0", FOR PRESSURE "1" ,/) $\operatorname{READ}(*, 1010)$ II

IF (II.EQ.1) GO TO 290

250 IF (IU.EQ.1)GO TO 260

1100 FORMAT(' ENTER A TEMPERATURE IN DEGREES F, ENTER 0 FOR RESTART',/) WRITE $(*, 1100)$

$\operatorname{READ}(*, *) \mathrm{TI}$

IF (TI.EQ.0.ODO) GO TO 110

$\mathrm{T}=(\mathrm{TI}-32 . \mathrm{DO}) / 1.8 \mathrm{DO}+273.15 \mathrm{DO}$

GO TO 270

260 WRITE $($ *, 1110)

1110 FORMAT(' ENTER A TEMPERATURE(R) ,ENTER O FOR RESTART',/) $\operatorname{READ}(*, *) \mathrm{T}$

270 IF (T.LT..000001D0) GO TO 110

IF (T.GT.TCC.OR.T.LT.TTP) GO TO 280

$\mathrm{P}=\mathrm{VPN}(\mathrm{T})$

IF (IP.EQ.0) P=P+.00001DO

IF (IP.EQ.1) $P=P-.00001 D 0$

$\mathrm{D}=\mathrm{FIND} \quad \mathrm{D}(\mathrm{P}, \mathrm{T})$

$\mathrm{PI}=\mathrm{P} \star .101325$

CALL RE PRO (PI, D , T, IU, IV, IC, IP, TF, TDEL)

GO TO 250

$280 \mathrm{X} 1=(\mathrm{TTP}-273.15 \mathrm{DO}) * 1.8 \mathrm{D} 0+32 . \mathrm{DO}$

$\mathrm{X} 2=(\mathrm{TCC}-273.15 \mathrm{DO}) \star 1.8 \mathrm{DO}+32 . \mathrm{DO}$

WRITE $(*, 1120) \mathrm{TTP}, \mathrm{TCC}, \mathrm{X} 1, \mathrm{X} 2$

1120 FORMAT(' FOR SATURATION ',F6.2,'< TEMP< ',F6.2,' $\mathrm{K}^{\prime}$, , /

$A$ ' OR ',E7.2,'<TEMP<',F7.2,' $\left.\mathrm{F}^{\prime}, / 1\right)$

GO TO 250

290 IF (IU.EQ.1) GO TO 300

WRITE $(\star, 1130)$

1130 FORMAT(' ENTER A PRESSURE IN LB/SQ IN 0 RESTRATS PROGRAM',/)

$\operatorname{READ}(*, *) \mathrm{P}$

$\mathrm{PI}=(\mathrm{P} / 14.695949 \mathrm{DO}) * .101325 \mathrm{D} 0$

$P=P I / .101325 D 0$

IF (PI.LE.O.ODO)GO TO 110

GO TO 310

300 WRITE $(*, 1140)$

1140 FORMAT(' ENTER A PRESSURE (MPA) O RESTARTS PROGRAM', /)

$\operatorname{READ}(*, *) \mathrm{PI}$

IF (PI.LE.0.ODO) GO TO 110

$\mathrm{P}=\mathrm{PI} / .101325$

310 IF (PI.GT.PCC.OR.PI.LT.PTP) GO TO 320

$T=F I N D \quad T V(P)$

$\mathrm{P}=\mathrm{VPN}(\mathrm{T})$

IF (IP.EQ.1) $\mathrm{P}=\mathrm{P}-.00001 \mathrm{DO}$

IF (IP.EQ.0) $\mathrm{P}=\mathrm{P}+.00001 \mathrm{DO}$

$\mathrm{D}=\mathrm{F}$ IND $\mathrm{D}(\mathrm{P}, \mathrm{T})$

$\mathrm{PI}=\mathrm{P}^{\star}$. 101325

CALL RE PRO(PI, D, T, IU, IV, IC, IP, TF, TDEL)

GO TO 290 
$320 \mathrm{PTPF}=\mathrm{PTP} * 14.695949 / .101325$

$\mathrm{PCCF}=\mathrm{PCC} * 14.695949 / .101325$

WRITE $(*, 1150)$ PTP, PCC, PTPF, PCCF

1150 FORMAT(' YOUR INPUT PRESSURE IS OUTSIDE THE RANGE OF SATURATION'

A ' PRESSURES' $/$ ' FOR THIS FLUID THE RANGE IS ',F6.5,' TO ',F6.3,

B' MPA,' $/$ ' OR ',F6.5,' TO ',F6.3,' PSIA'/' TRY AGAIN', /) GO TO 290

330 IF (IC.EQ.1) GO TO 370

IF (IU.EQ.1) GO TO 340

WRITE $(*, 1160)$

1160 FORMAT(' ENTER A STARTING TEMPERATURE, A FINAL TEMPERATURE'

A $/$ AND A TEMPERATURE INCREMENT, IN DEGREES F AND IN THAT ORDER'/

B ' ENTER $0,0,0$ TO RESTART'/)

$\operatorname{READ}(*, *) T S, T F, T D E L$

IF (TDEL.LE.O.ODO) GO TO 110

$\mathrm{TS}=(\mathrm{TS}-32 . \mathrm{D} 0) / 1.8 \mathrm{D} 0+273.15 \mathrm{D} 0$

$\mathrm{TF}=(\mathrm{TF}-32 . \mathrm{D} 0) / 1.8 \mathrm{D} 0+273.15 \mathrm{D} 0$

TDEL=TDEL/1.8DO

IF (TS.LT.TTP.OR.TS.GT.TCC) GO TO 360

IF (TF.LT.TTP.OR.TF.GT.TCC) GO TO 360

GO TO 350

340 WRITE $(*, 1170)$

1170 FORMAT(' ENTER A STARTING TEMPERATURE, A FINAL TEMPERATURE'

$1 / '$ AND A TEMPERATURE INCREMENT IN KELVINS AND IN THAT ORDER',/

$2^{\prime}$ ENTER $0,0,0$ TO RESTART PROGRAM' /)

$\operatorname{READ}(*, *) T S, T F, T D E L$

IF (TDEL.LE.0.ODO) GO TO 110

IF (TS.LT.TTP.OR.TS.GT.TCC) GO TO 360

$350 \mathrm{~T}=\mathrm{TS}$

IF (TF. LT.TTP.OR.TF.GT.TCC) GO TO 360

$\mathrm{P}=\mathrm{VPN}(\mathrm{T})$

IF (IP.EQ.0) $P=P+.00001 D 0$

IF (IP.EQ.1) $\mathrm{P}=\mathrm{P}-.00001 \mathrm{DO}$

$\mathrm{D}=\mathrm{F}$ IND $\mathrm{D}(\mathrm{P}, \mathrm{T})$

$P I=P * .101325$

CALL RE PRO(PI, D, T, IU, IV, IC, IP, TF , TDEL)

GO TO 330

$360 \times 1=(T T P-273 \cdot 15 \mathrm{DO}) * 1.8 \mathrm{D} 0+32 . \mathrm{DO}$

$\mathrm{X} 2=(\mathrm{TCC}-273.15 \mathrm{DO}) * 1.8 \mathrm{D} 0+32 . \mathrm{D} 0$

WRITE $(*, 1180)$ TTP , TCC , X1, X2

1180 FORMAT(' FOR SATURATION, ',F6.2,'< TEMP< ',F6.2, ' $\mathrm{K}$ ', /,

A , 13X,'OR, ',F7.1,' 'TEMP<',F7.1,' F. TRY AGAIN.', /) GO TO 330

370 IF(IU.EQ.1) GO TO 380

WRITE $(*, 1190)$

1190 FORMAT(' ENTER PRESSURE(PSIA)， STARTING TEMPERATURE(F), FINAL '

A 'TEMPERATURE (F)' $/$ ' AND A TEMPERATURE INCREMENT, IN THAT ORDER', /

B ' ENTER $0,0,0,0$ TO RESTART PROGRAM' $/)$

$\operatorname{READ}(*, *) \mathrm{P}, \mathrm{TS}, \mathrm{TF}, \mathrm{TDEL}$

IF (TDEL. LE. 0.ODO) GO TO 110

$P I=(P / 14.695949 D 0) * .101325 D 0$

$\mathrm{T}=(\mathrm{TS}-32 . \mathrm{DO}) / 1.8 \mathrm{D} 0+273.15 \mathrm{D} 0$

$\mathrm{TF}=(\mathrm{TF}-32 . \mathrm{DO}) / 1.8 \mathrm{D} 0+273.15 \mathrm{D} 0$

TDEL $=$ TDEL $/ 1.8 D 0$

$\mathrm{P}=\mathrm{PI} / .101325$

CALL LIMITS (PI, T, IL)

IF (IL.LE.0) GO TO 370

CALL LIMITS (PI, TF, IL)

IF(IL.LE.O)GO TO 370

GO TO 390

380 WRITE $(*, 1200)$

1200 FORMAT(' ENTER A PRESSURE(MPA), A STARTING 'TEMPERATURE(K), A ' 
1'FINAL TEMPERATURE AND A' $/$ ' TEMPERATURE INCREMENT'

2' AND IN THAT ORDER, TO RESTART PROGRAM ENTER $" 0,0,0,0 " 1,1)$

$\operatorname{READ}(*, *) \mathrm{PI}, \mathrm{TS}, \mathrm{TF}, \mathrm{TDEL}$

IF (TDEL.LE.O.ODO) GO TO 110

$\mathrm{T}=\mathrm{TS}$

$\mathrm{P}=\mathrm{PI} / .101325$

CALL LIMITS ( PI, T, IL)

IF (IL.LE.0)GO TO 370

CALL LIMITS (PI,TF, IL)

IF (IL.LE.O)GO TO 370

$390 \mathrm{D}=\mathrm{F}$ IND $\mathrm{D}(\mathrm{P}, \mathrm{T})$

CALL RE PRO(PI,D, T, IU, IV, IC, IP, TF , TDEL)

GO TO 370

110 CONTINUE

WRITE $(*, 1210)$

1210 FORMAT(//' FOR MORE PROPERTIES ENTER 0, TO TERMINATE EINTER 1')

$\operatorname{READ}(*, 1010)$ IM

IF (IM.EQ.0)GO TO 1

999 CONTINUE

STOP

END

SUBROUTINE REPRO( $P I, D, T, I U, I V, I C, I P, T F, T D E L)$

IMPLICIT REAL* $8(\mathrm{~A}-\mathrm{H})$

IMPLICIT REAL *8 $(O-Z)$

IMPLICIT INTEGER * $4(I-N)$

$\mathrm{N}=500$

$E M=4.0026$

$\mathrm{P}=\mathrm{PI} / .101325$

IF (IV.EQ.0) TF $=\mathrm{T}-1 . \mathrm{DO}$

IF (IU.EQ.0) GO TO 100

$\operatorname{WRITE}(*, 1000)$

WRITE $(*, 1010)$

WRITE $(*, 1020)$

GO TO 110

$100 \operatorname{WRITE}(*, 1000)$

WRITE $(*, 1030)$

$\operatorname{WRITE}(*, 1040)$

110 CONTINUE

DO $210 \quad I=1, N$

IF (I.EQ.1)GO TO 120

$D=F I N D \quad D(P, T)$

$120 \mathrm{H}=\operatorname{ENTHAL}(\mathrm{P}, \mathrm{D}, \mathrm{T})$

$E=H-101.325 D 0 * P / D$

$S=E N T R O P(D, T)$

$\mathrm{W}=\operatorname{SOUND}(\mathrm{D}, \mathrm{T})$

$\mathrm{CPP}=\mathrm{CP}(\mathrm{D}, \mathrm{T})$

$C V V=C V(D, T)$

$150 \mathrm{~V}=\mathrm{VISC}(\mathrm{D}, \mathrm{T})$

$T H=T H E R M(D, T) * 100 . D 0$

EPS $=$ FDIEL $(D)$

IF (IU.EQ.0)GO TO 160

$\mathrm{PO}=\mathrm{P} * .101325$

WRITE $(*, 2030)$ PO, T, D , E , H, S , CVV , CPP , W, V , TH, EPS

GO TO 200

$160 \mathrm{H}=\mathrm{H} /(2.324445 \mathrm{DO} * \mathrm{EM})$

$E=E /(2.324445 D 0 * E M)$

$\mathrm{S}=\mathrm{S} /(4.184001 \mathrm{DO} * \mathrm{EM})$

$\mathrm{CPP}=\mathrm{CPP} /(4.184001 \mathrm{DO}$ *EM)

$\mathrm{CVV}=\mathrm{CVV} /(4.184001 \mathrm{DO} * \mathrm{EM})$

$\mathrm{W}=\mathrm{W} * 3.280840 \mathrm{D} 0$

$\mathrm{PO}=\mathrm{P} \star 14.695949 \mathrm{DO}$

$\mathrm{DO}=\mathrm{D} \star \mathrm{EM} / 16.01846371 \mathrm{D} 0$ 
$\mathrm{TO}=\mathrm{T} \star 1.8 \mathrm{DO}-459.67 \mathrm{DO}$

$190 \mathrm{~V}=\operatorname{VISC}(\mathrm{D}, \mathrm{T}) * .0067196897 \mathrm{D} 0$

$\mathrm{TH}=\mathrm{THERM}(\mathrm{D}, \mathrm{T}) * .000578176 \mathrm{DO}$

$E P S=F D I E L(P, D, T)$

WRITE $(*, 3030)$ PO, TO,DO,E,H, S, CVV, CPP, W, V, TH, EPS

$200 \mathrm{~T}=\mathrm{T}+\mathrm{TDEL}$

IF (T.GT.TF+.01DO) GO TO 220

IF (IC.NE.O) GO TO 210

$\mathrm{P}=\mathrm{VPN}(\mathrm{T})$

IF (IP.EQ.0) P $=\mathrm{P}+.00001 \mathrm{DO}$

IF (IP.EQ.1) $\mathrm{P}=\mathrm{P}-.00001 \mathrm{D} 0$

210 CONTINUE

220 CONTINUE

WRITE $(*, 1000)$

1000 FORMAT(' ')

RETURN

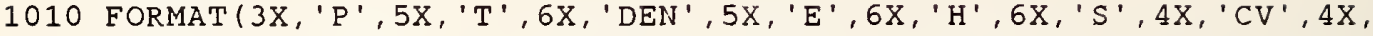

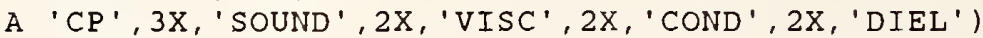

1020 FORMAT $\left(3 \mathrm{X},{ }^{\prime} \mathrm{MPA}{ }^{\prime}, 3 \mathrm{X},{ }^{\prime} \mathrm{K}{ }^{\prime}, 6 \mathrm{X},{ }^{\prime} \mathrm{MOL} / \mathrm{L}{ }^{\prime}, 2 \mathrm{X},{ }^{\prime}===\mathrm{J} / \mathrm{MOL}=={ }^{\prime}, 2 \mathrm{X},{ }^{\prime}====\right.$

A ' $\left.J / M O L-K===', 2 X, ' M / S^{\prime}, 3 X,{ }^{\prime} P A-S^{\prime}, 1 X, ' M W / M-K ', 2 X,{ }^{\prime}==\prime^{\prime}, /, 61 X,{ }^{\prime} E+6^{\prime}\right)$

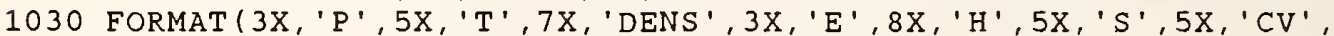

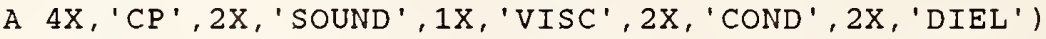

1040 FORMAT (3X, 'PSIA', $2 \mathrm{X},{ }^{\prime} \mathrm{F}^{\prime}, 7 \mathrm{X},{ }^{\prime} \mathrm{LB} /{ }^{\prime}, 3 \mathrm{X},{ }^{\prime}====\mathrm{BTU} /===\mathrm{X}^{\prime}, 1 \mathrm{X},{ }^{\prime}======\mathbf{x}^{\prime}$,

$\mathrm{A}, \mathrm{BTU} /===={ }^{\prime}, 2 \mathrm{X},{ }^{\prime} \mathrm{F} / \mathrm{S}^{\prime}, 3 \mathrm{X},{ }^{\prime} \mathrm{LB} /{ }^{\prime}, 2 \mathrm{X},{ }^{\prime} \mathrm{BTU} / \mathrm{\prime}^{\prime}, 2 \mathrm{X},{ }^{\prime}==^{\prime}, /, 17 \mathrm{X}, \mathrm{CU}^{\mathrm{FT}}$, ,

$\left.\mathrm{B} 6 \mathrm{X},{ }^{\prime} \mathrm{LB} \mathrm{B}^{\prime}, 14 \mathrm{X},{ }^{\prime} \mathrm{LB}-\mathrm{F}^{\prime}, 13 \mathrm{X},{ }^{\prime} \mathrm{FT}-\mathrm{S}^{\prime}, 1 \mathrm{X},{ }^{\prime} \mathrm{FT}-\mathrm{HR}-\mathrm{F}^{\prime}, /, 62 \mathrm{X}, \mathrm{C}^{\prime} \mathrm{E}+5^{\prime}\right)$

2030 FORMAT (F8.3,F7.2,F7.3,2F7.0,F6.1,F5.1,F6.1,F6.0,F6.1,F6.1,F8.5)

3030 FORMAT (F8.1,F7.2,F7.3,2F7.1,3F6.3,F6.0,F5.2,F6.4,F 8.5)

END

SUBROUTINE INFO

WRITE $(*, 101)$

101 FORMAT('

A

1 .

$2^{\prime}$

$3^{\prime}$

$4^{\prime}$

$5^{\prime}$

$A^{\prime}$

$6^{\prime}$

WRITE $(*, 100)$

\author{
WRITTEN BY'// \\ ROBERT D. MCCARTY'/ \\ THERMOPHYSICS DIVISION'/ \\ CENTER FOR CHEMICAL ENGINEERING'/ \\ NATIONAL BUREAU OF STANDARDS'/ \\ BOULDER, COLORADO'// \\ DISTRIBUTED BY'// \\ THE OFFICE OF STANDARD REFERENCE DATA'/ \\ NATIONAL BUREAU OF STANDARDS, WASHINGTON, DC'/)
}

100 FORMAT(/ ' WHEN THE PROGRAM ASKS FOR A PRESSURE, DENSITY AND'

* ' TEMPERATURE, ' / ' ENTER ANY TWO AND A ZERO FOR THE THIRD.' /

* ' to terminate the PROGRAM ENTER ZERO For ALL THREE.'/)

RETURN

END 
SUBROUTINE FDATA(IF)

IMPLICIT REAL $\star 8(A-H)$

IMPLICIT REAL * $8(0-Z)$

IMPLICIT INTEGER* $4(\mathrm{I}-\mathrm{N})$

DIMENSION $B(27,3)$

COMMON/COEF / B

COMMON/LIM/TUL, TLL , PUL , TCC , DCC , PCC

$\operatorname{OPEN}\left(5\right.$, FILE $=$ ' HELIUM. $\mathrm{COF}^{\prime}$ )

DO $60 \mathrm{~J}=1,3$

DO $60 \quad I=1,27$

$60 \operatorname{READ}(5,101) \mathrm{B}(I, J)$

101 FORMAT (D20.13)

READ $(5,102)$ TUL , TLL , PUL , TCC, DCC , PCC

CLOSE ( 5 , STATUS = ' KEEP')

102 FORMAT (F10.8, E14.8, 3F 8.2, 2F 8.4)

RETURN

END

SUBROUTINE LIMITS (PI, T, IL)

IMPLICIT REAL * $8(\mathrm{~A}-\mathrm{H})$

IMPLICIT REAL $* 8(0-Z)$

IMPLICIT INTEGER * $4(I-N)$

COMMON/LIM/TUL, TLL, PUL, TCC, DCC , PCC

$\mathrm{P}=\mathrm{PI} / .101325$

IF (PI.GT. PUL) GO TO 10

IF (T . GT. TUL.OR.T . LT. TLL)GO TO 12

PM=PMELT $(T)$

IF (P . GT. PM) GO TO 20

$I L=1$

RETURN

10 PULF $=$ PUL $\star 14.6959496 / .101325$

WRITE $(*, 11)$ PUL , PULF

11 FORMAT(' THE INPUT PRESSURE IS OUT OF THE RANGE OF THIS EQUATION '

1/' THE RANGE FOR THIS EQUATION IS FROM 0 TO ',F6.0,' MPA'

$2 /$

$I L=0$

OR ',F7.0', PSIA')

RETURN

$12 \mathrm{TLLF}=(\mathrm{TLL}-273 \cdot 15 \mathrm{D} 0) * 1.8 \mathrm{D} 0+32 . \mathrm{D} 0$

TULF $=($ TUL $-273.15 \mathrm{DO}) * 1.8 \mathrm{D} 0+32 . \mathrm{DO}$

WRITE $(*, 13)$ TLL, TUL, TLLF, TULF

13 FORMAT(' THE INPUT TEMPERATURE IS OUT OF RANGE'

A $/$ THE RANGE FOR THIS EQUATION IS ', F6.2,' $\mathrm{K}$ TO ', F6.0,' $\mathrm{K}$ ', /,

B $27 X^{\prime}{ }^{\prime}$ OR ', F8.2,' $F$ TO ',F6.0,' F')

$\mathrm{IL}=0$

RETURN

20 TM=TMELT ( $P$ )

$\mathrm{TF}=(\mathrm{TM}-273.15 \mathrm{D} 0) * 1.8 \mathrm{D} 0+32 . \mathrm{D} 0$

WRITE $(*, 22)$ TM, TF

22 FORMAT(' SOLID PHASE DETECTED.', /,' FOR THIS PRESSURE, TEMP'

A ' SHOULD EXCEED ', F 8.3,' K, OR', F9.3,' $\mathrm{F}^{\prime}$ ')

$I L=0$

END

DOUBLE PRECISION FUNCTION CV $(\mathrm{D}, \mathrm{T})$

IMPLICIT REAL $\star 8(A-H)$

IMPLICIT REAL $* 8(0-\mathrm{Z})$

IMPLICIT INTEGER * $4(I-N)$

C CALCULATES CV $(J /(M O L * K))$. INPUT DENS(MOL/L) AND TEMP(K).

CALL PROPS ( PP, D, T, 6)

$\mathrm{CV}=\mathrm{PP}$

END

DOUBLE PRECISION FUNCTION ENTHAL $(P, D, T)$

IMPLICIT REAL* $8(A-H)$

IMPLICIT REAL $8(0-Z)$

IMPLICIT INTEGER* $4(I-N)$ 


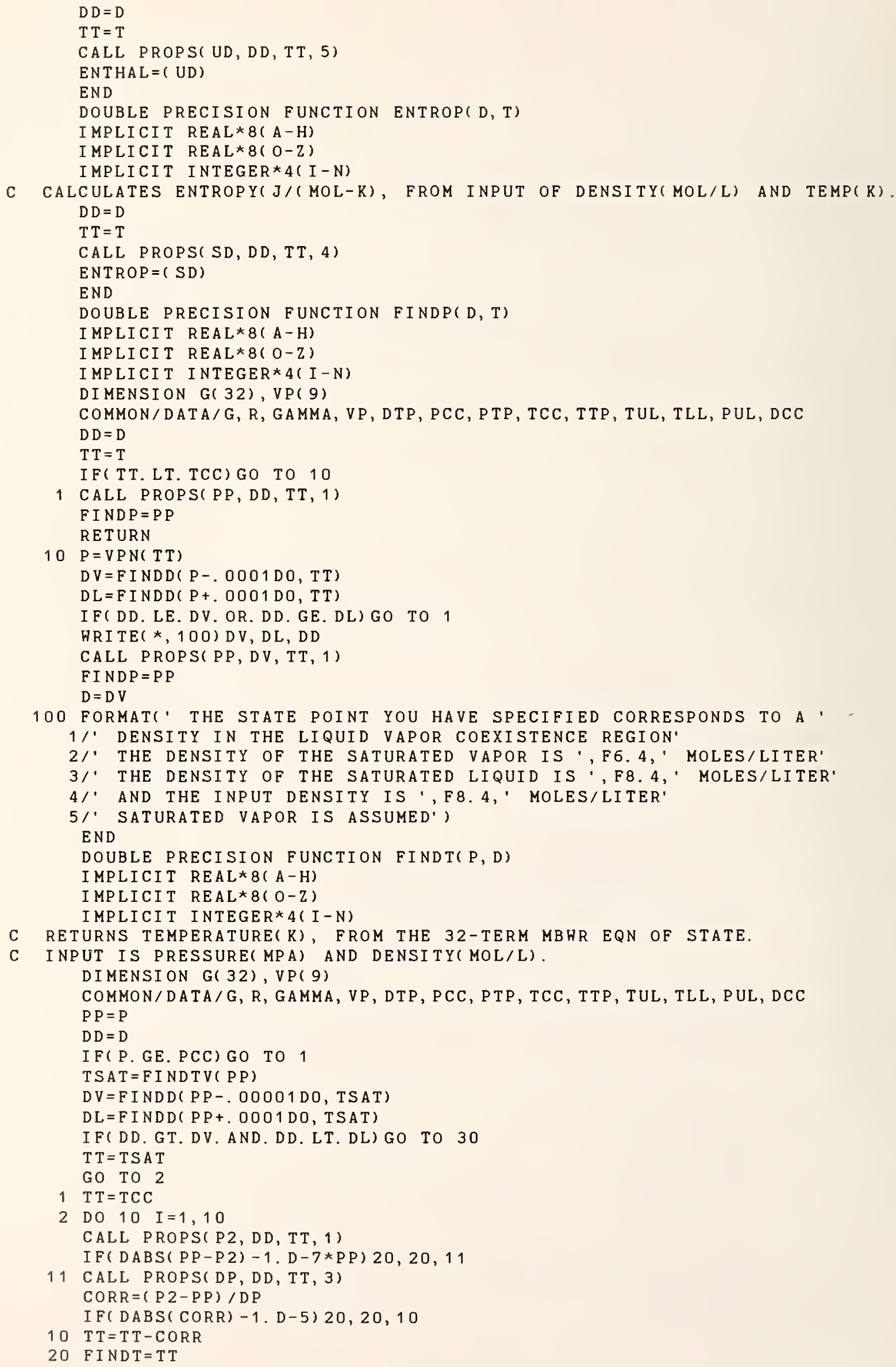




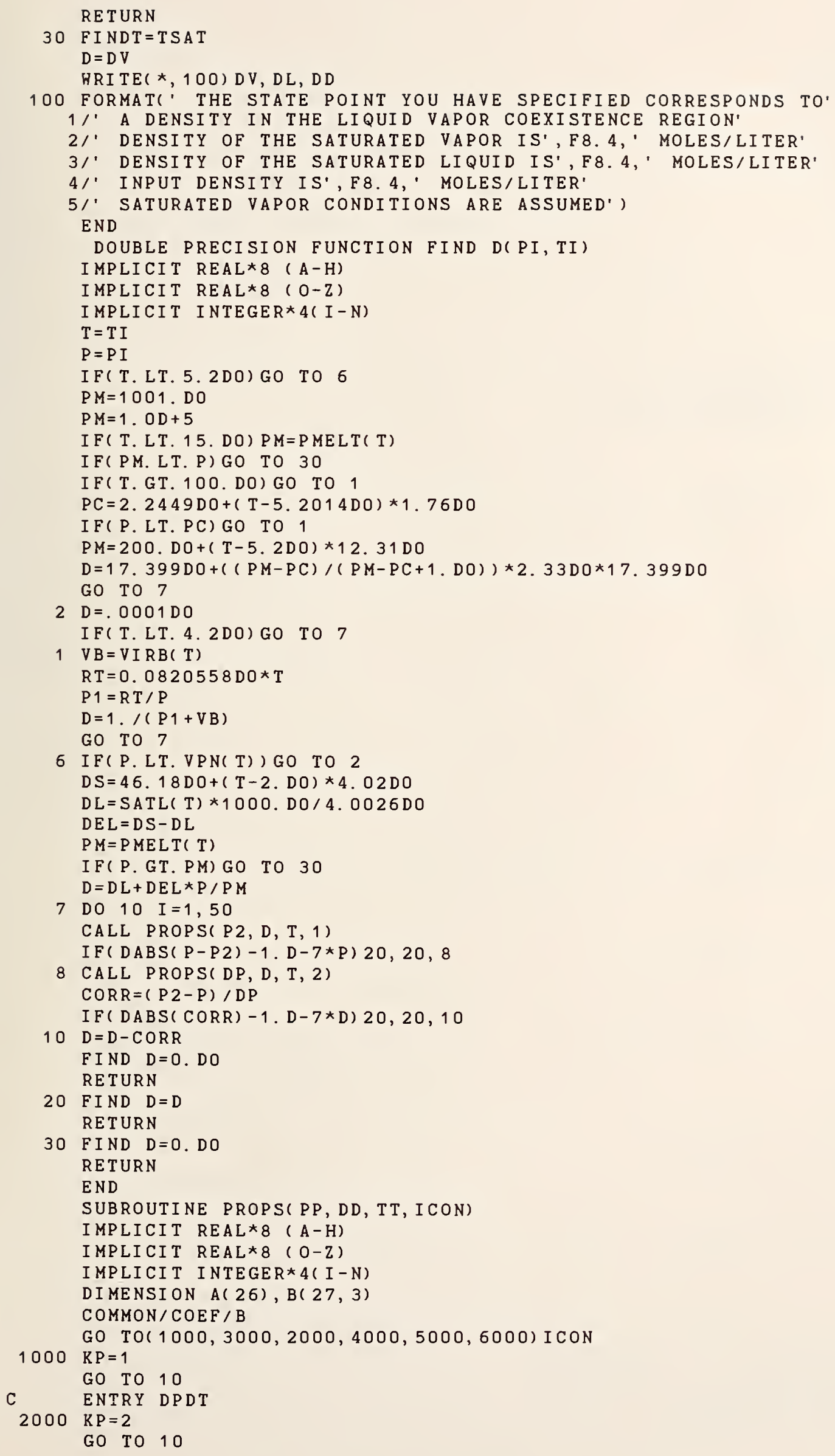




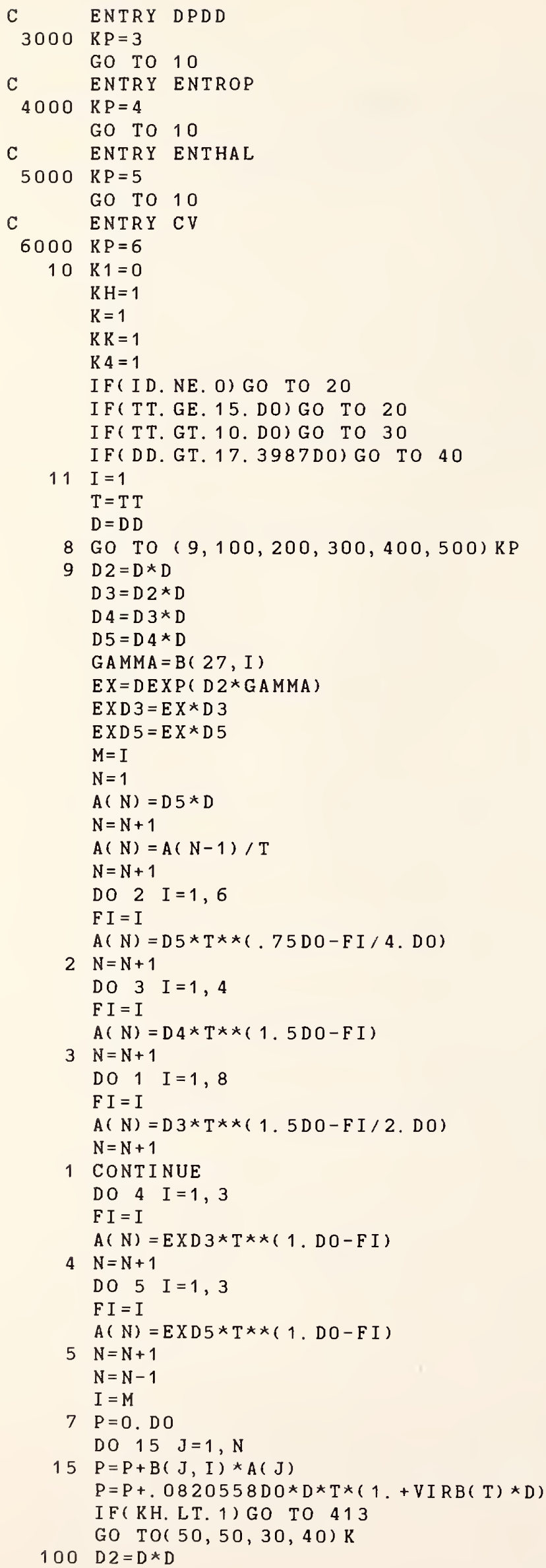


D $3=D \star \star 3$

$\mathrm{D} 4=\mathrm{D} 3 \star \mathrm{D}$

$\mathrm{D} 5=\mathrm{D} 4 * \mathrm{D}$

$\mathrm{D} 6=\mathrm{D} 5 \star \mathrm{D}$

$\mathrm{T} 2=\mathrm{T} \star \mathrm{T}$

$\mathrm{T} 3=\mathrm{T} 2 \star \mathrm{T}$

$\mathrm{T} 4=\mathrm{T} \star \star 4$

$M=I$

$G A M M A=B(27, M)$

$E X=D E X P(D 2$ * GAMMA $)$

$\mathrm{N}=1$

$\mathrm{R}=.0820558 \mathrm{DO}$

$A(N)=0.0 D 0$

$\mathrm{N}=\mathrm{N}+1$

$A(N)=(-1 . D 0) \star D 6 / T 2$

$\mathrm{N}=\mathrm{N}+1$

DO $102 I=1,6$

$F I=I$

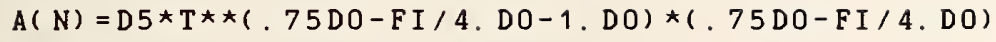

$102 \mathrm{~N}=\mathrm{N}+1$

DO $103 \quad I=1,4$

$\mathrm{FI}=\mathrm{I}$

$A(N)=D 4 * T \star \star(1.5 D 0-F I / 1 . D 0-1 . D 0) \star(1.5 D 0-F I)$

$103 \mathrm{~N}=\mathrm{N}+1$

DO $101 \mathrm{I}=1,8$

$\mathrm{FI}=\mathrm{I}$

$A(N)=D 3 \star T \star \star(1.5 D O-F I / 2 . D O-1 . D O) \star(1.5 D O-F I / 2 . D O)$

$101 \mathrm{~N}=\mathrm{N}+1$

DO $104 I=1,3$

$\mathrm{FI}=\mathrm{I}$

$A(N)=E X \star D 3 \star T \star \star(1 . D O-F I-1 . D 0) \star(1 . D O-F I)$

$104 \mathrm{~N}=\mathrm{N}+1$

DO $105 \mathrm{I}=1,3$

$\mathrm{FI}=\mathrm{I}$

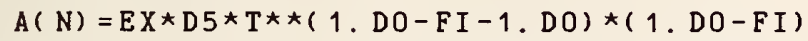

$105 \mathrm{~N}=\mathrm{N}+1$

$\mathrm{N}=\mathrm{N}-1$

$\mathrm{P}=0$

DO $115 \mathrm{~J}=1, \mathrm{~N}$

$115 P=P+A(J) \star B(J, M)$

$P=P+R \star D \star(1 . D O+V I R B(T) \star D)+R \star D \star T \star D B D T(T) \star D$

$I=M$

GO TO( $50,50,30,40) \mathrm{K}$

$200 \mathrm{D} 2=\mathrm{D} \star \mathrm{D}$

$D 3=D 2 \star D$

$D 4=D 3 * D$

$D 5=D 4 \star D$

$M=I$

$G A M M A=B(27, M)$

$\mathrm{EX}=\mathrm{DEXP}(\mathrm{D} 2$ * GAMMA)

$D E X=G A M M A \star 2 . D O \star D \star E X$

$\mathrm{N}=1$

$\mathrm{R}=0.0820558 \mathrm{DO}$

$A(N)=6$. DO $D 5$

$\mathrm{N}=\mathrm{N}+1$

$A(N)=A(N-1) / T$

$\mathrm{N}=\mathrm{N}+1$

DO $202 I=1,6$

$\mathrm{FI}=\mathrm{I}$

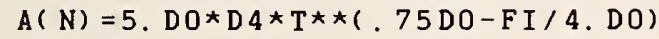

$202 \mathrm{~N}=\mathrm{N}+1$

DO $203 \mathrm{I}=1,4$

$\mathrm{FI}=\mathrm{I}$

$A(N)=D 3 \star 4 . D 0 \star T \star \star(1.5 D O-F I)$

$203 \mathrm{~N}=\mathrm{N}+1$

DO $201 \quad I=1,8$

$\mathrm{FI}=\mathrm{I}$

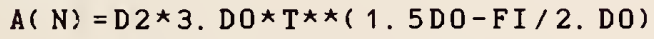




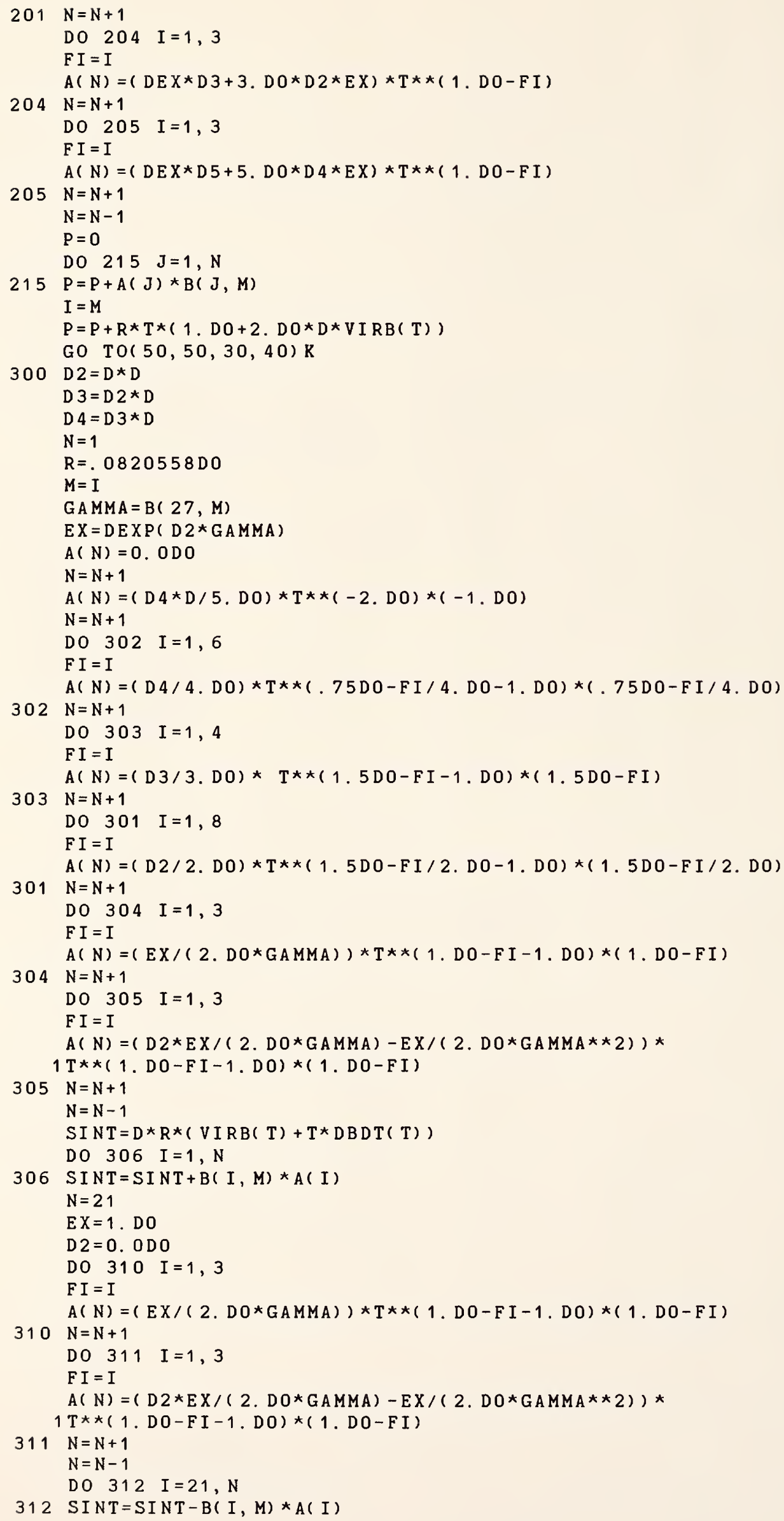


$P=(9.371658 D 0+5.193043 D 0 \star D L O G(T / 4.2144 D 0)-25.31469 D 0$

$1 \star(S I N T+R * D L O G(R \star T \star D)))$

$\mathrm{P}=\mathrm{P} \star 4.0026 \mathrm{DO}$

$I=M$

GO TO $50,50,30,40) \mathrm{K}$

$400 \mathrm{KH}=0$

GO TO 9

$413 \mathrm{PP}=\mathrm{P}$

$\mathrm{KH}=1$

D $2=D * D$

$\mathrm{D} 3=\mathrm{D} * \mathrm{D} 2$

D $4=\mathrm{D} 3 * \mathrm{D}$

$\mathrm{N}=1$

$\mathrm{R}=.0820558 \mathrm{DO}$

$\mathrm{M}=\mathrm{I}$

$G A M M A=B(27, M)$

$\mathrm{EX}=\mathrm{DEXP}(\mathrm{D} 2$ * GAMMA)

$A(N)=(D 4 * D) / 5$. D 0

$\mathrm{N}=\mathrm{N}+1$

$A(N)=(D 4 * D / 5, D 0) *(2, D 0 / T)$

$\mathrm{N}=\mathrm{N}+1$

DO $402 \quad I=1,6$

$\mathrm{F} I=\mathrm{I}$

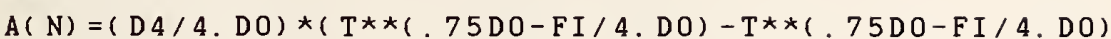

$1 \star(.75 \mathrm{DO}-\mathrm{FI} / 4 . \mathrm{DO}))$

$402 \mathrm{~N}=\mathrm{N}+1$

DO $403 \quad I=1,4$

$\mathrm{FI}=\mathrm{I}$

$A(N)=(D 3 / 3, D 0) *(T \star \star(1.5 D 0-F I)-T \star \star(1.5 D 0-F I) \star(1.5 D 0-F I))$

$403 \mathrm{~N}=\mathrm{N}+1$

DO $401 \quad I=1,8$

$\mathrm{F} I=\mathrm{I}$

$A(N)=(D 2 / 2 . D 0) \star(T \star \star(1.5 D O-F I / 2, D 0)-T \star \star(1,5 D 0-F I / 2 . D 0)$

$1 \star(1.5 \mathrm{DO}-\mathrm{FI} / 2 . \mathrm{DO}))$

$401 \mathrm{~N}=\mathrm{N}+1$

DO $404 \quad I=1,3$

$\mathrm{F} I=\mathrm{I}$

$A(N)=(E X /(2, D 0 * G A M M A)) \star(T \star \star(1, D O-F I)-T \star \star(1, D 0-F I) \star(1 . D 0-F I))$

$404 \mathrm{~N}=\mathrm{N}+1$

DO $405 \mathrm{I}=1,3$

$\mathrm{F} I=\mathrm{I}$

$A(N)=(D 2 \star E X /(2 . D O \star G A M M A)-E X /(2 . D 0 \star G A M M A \star \star 2))$

$1 \star(T \star \star(1 . D O-F I)-T \star \star(1 . D O-F I) \star(1 . D O-F I))$

$405 \mathrm{~N}=\mathrm{N}+1$

$\mathrm{N}=\mathrm{N}-1$

$\mathrm{HINT}=\mathrm{R} \star \mathrm{T} \star \mathrm{T} \star \mathrm{D} \star \mathrm{DBDT}(\mathrm{T}) \quad \star(-1, \mathrm{DO})$

DO $406 I=1, N$

406 HINT $=H I N T+B(I, M) \star A(I)$

$\mathrm{N}=21$

$\mathrm{D} 2=0.0 \mathrm{DO}$

$\mathrm{EX}=1 . \mathrm{DO}$

DO $410 \quad I=1,3$

$\mathrm{F} I=\mathrm{I}$

$A(N)=(E X /(2 . D O \star G A M M A)) \star(T \star \star(1 . D O-F I)-T \star \star(1 . D O-F I) \star(1 . D O-F I))$

$410 \mathrm{~N}=\mathrm{N}+1$

DO $411 \mathrm{I}=1,3$

$\mathrm{F} I=\mathrm{I}$

$A(N)=(D 2 \star E X /(2 . D 0 \star G A M M A)-E X /(2 . D 0 \star G A M M A \star \star 2)) \star(T \star \star(1 . D O-F I)$

$1-\mathrm{T} * \star(1 . \mathrm{DO}-\mathrm{FI}) \star(1 .-\mathrm{FI}))$

$411 \mathrm{~N}=\mathrm{N}+1$

$\mathrm{N}=\mathrm{N}-1$

DO $412 I=21, \mathrm{~N}$

$412 \mathrm{HINT}=\mathrm{HINT}-\mathrm{B}(\mathrm{I}, \mathrm{M}) \star \mathrm{A}(\mathrm{I})$

$\mathrm{P}=21.8228 \mathrm{DO}+5.193043 \mathrm{DO} *(\mathrm{~T}-4.2144 \mathrm{D} 0)+25.31469 \mathrm{D} 0$

$1 \star(\mathrm{HINT}+\mathrm{PP} / \mathrm{D}-\mathrm{R} \star \mathrm{T})$

$\mathrm{P}=\mathrm{P} \star 4.0026 \mathrm{DO}$

$I=M$

GO TO $(50,50,30,40) \mathrm{K}$ 


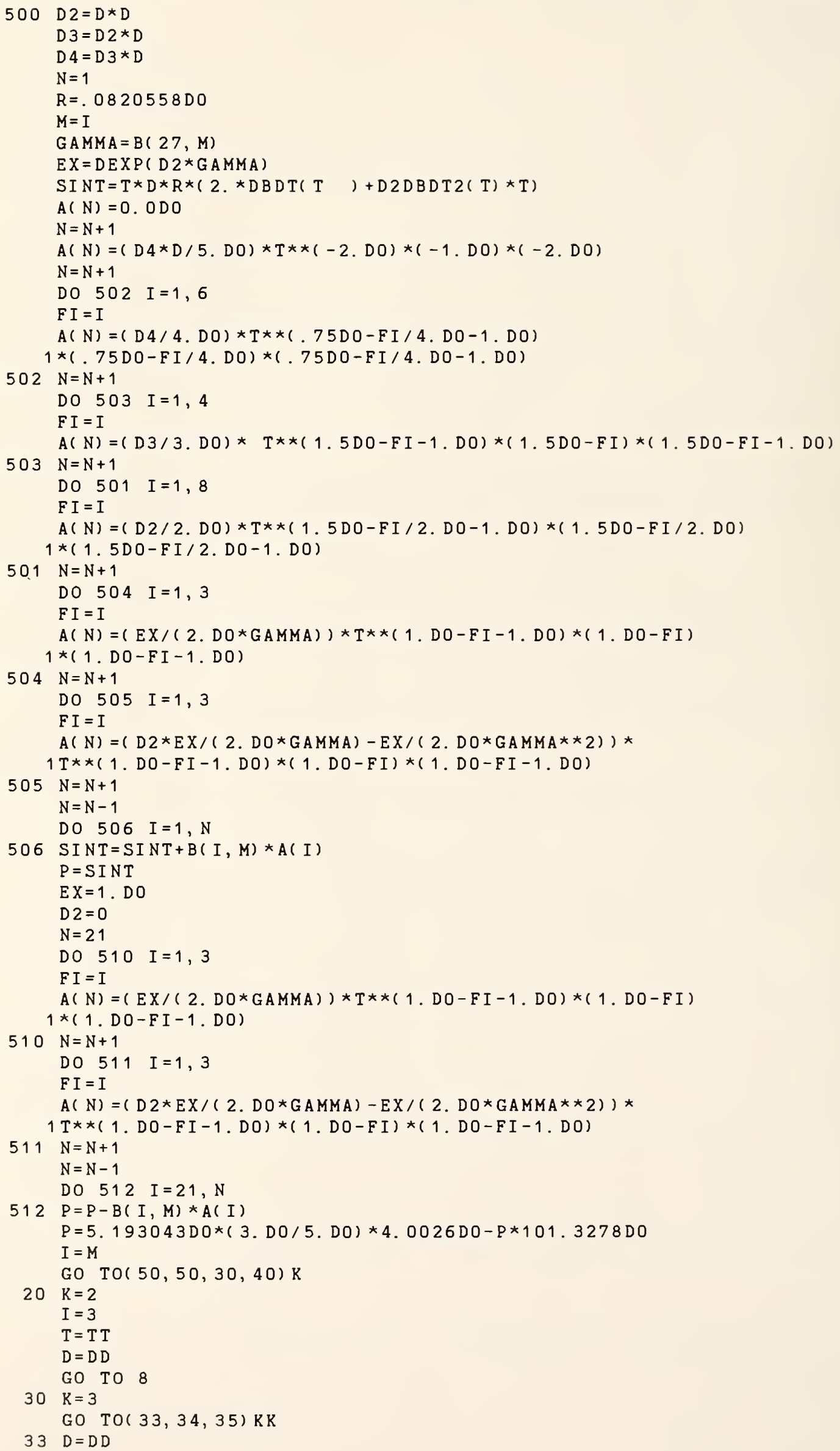


$\mathrm{T}=\mathrm{T} \mathrm{T}$

$\mathrm{KK}=2$

IF( DD. GT. 17. 3987DO) GO TO 40

$I=1$

GO TO 8

34 P TI $=$ P

$I=3$

$\mathrm{KK}=3$

$\mathrm{T}=\mathrm{T} \mathrm{T}$

$\mathrm{D}=\mathrm{DD}$

GO TO 8

35 P TII I = P

$38 \mathrm{~F}=(15 . \mathrm{DO}-\mathrm{T}) / 5$. DO

$\mathrm{P}=\mathrm{F} \star \mathrm{PTI}+(1 . \mathrm{DO}-\mathrm{F}) \star \mathrm{PTI} I$

IF( KH. LT. 1) GO TO 413

$\mathrm{PP}=\mathrm{P}$

RETURN

40 I F ( K. EQ. 3) K $1=3$

GO $\mathrm{TO}(41,42,43,44) \mathrm{K} 4$

$41 \mathrm{~K}=4$

$\mathrm{I}=2$

$\mathrm{K} 4=2$

$\mathrm{D}=\mathrm{D} \mathrm{D}$

I F ( K1 . EQ. O ) T $=\mathrm{TT}$

GO TO 8

42 PII D $=$ P

$D=17.3987 \mathrm{DO}$

IF ( T. LT. 5. $2014 \mathrm{DO}) \mathrm{D}=\mathrm{SATL}(\mathrm{T}) \star 1000 . \mathrm{D} 0 / 4.0026 \mathrm{DO}$

$\mathrm{K} 4=3$

GO TO 8

43 PII DC $=$ P

$I=1$

$\mathrm{K} 4=4$

GO TO 8

44 PI DC $=$ P

$P=P I D C+(P I I D-P I I D C)$

$\mathrm{K} 4=1$

IF (K1.EQ. 3) GO TO 30

$P P=P$

RE TURN

$50 \quad P P=P$

RETURN

END

DOUBLE PRECISION FUNCTION CP $(D, T)$

I MPLICIT REAL*8 $(\mathrm{A}-\mathrm{H})$

IMPLICIT REAL*8 $(0-Z)$

IMPLICIT INTEGER* $4(\mathrm{I}-\mathrm{N})$

CALL PROPS( DPDD, D, T, 2)

CALL PROPS( DPDT, D, T, 3)

$C P=C V(D, T)+(T \star(D P D T \star \star 2) /((D \star \star 2) \star D P D D)) \star 101.3278 D 0$

RETURN.

END

DOUBLE PRECISIONFUNCTION $\operatorname{SOUND}(\mathrm{D}, \mathrm{T})$

IMPLICIT REAL* $8(\mathrm{~A}-\mathrm{H})$

I MPLICIT REAL*8 $(0-Z)$

IMPLICIT INTEGER* $4(\mathrm{I}-\mathrm{N})$

CALL PROPS( DPDD, D, T, 2)

SOUND $=((C P(D, T) / C V(D, T)) \star(D P D D \star 25311 . D O)) \star \star .5 D 0$

RETURN

END

DOUBLE PRECISION FUNCTION P MELT(TT)

I MPLICIT REAL* $8(\mathrm{~A}-\mathrm{H})$

IMPLICIT REAL*8 $(0-Z)$

I MPLICIT INTEGER*4( I-N)

DIMENSION A( 5 )

DATA A/33.28D0, $-44.156 \mathrm{DO}, 31.799 \mathrm{DO},-4.8159 \mathrm{DO}, .30313 \mathrm{DO} /$

$\mathrm{T}=\mathrm{T} \mathrm{T}$

IF (T. LE. 5. 2DO) GO TO 7 
$\mathrm{PMELT}=-17.80 \mathrm{DO}+17.31457 \mathrm{DO} \star \mathrm{T} \star \star 1.555414 \mathrm{DO}$

PMELT $=$ PMELT*. $98066 \mathrm{DO} / 1.01325 \mathrm{DO}$

RETURN

$7 \quad \mathrm{P}=0.0 \mathrm{DO}$

DO $9 I=1,5$

$9 \mathrm{P}=\mathrm{P}+\mathrm{A}(\mathrm{I}) \star \mathrm{T} \star \star(\mathrm{I}-1)$

PMELT $=P \star 9.80665 \mathrm{DO} / 10.1325 \mathrm{DO}$

RETURN

END

DOUBLE PRECISION FUNCTION VIRB(T)

IMPLICIT REAL*8 (A-H)

IMPLICIT REAL $* 8 \quad(0-Z)$

IMPLICIT INTEGER* $4(\mathrm{I}-\mathrm{N})$

DIMENSION A( 9)

DATA A $/-5.0815710041 \mathrm{D}-7,-1.1168680862 \mathrm{D}-4,1.1652480354 \mathrm{D}-2$,

$17.4474587998 \mathrm{D}-2,-5.3143174768 \mathrm{D}-1,-9.5759219306 \mathrm{D}-1$,

$23.9374414843 \mathrm{DO},-5.1370239224 \mathrm{DO}, 2$. $0804456338 \mathrm{DO} /$

$\mathrm{C}$ COEFFICIENTS FROM PROGRAM 5/28/70-1630

THIS SUB PROGRAM CALCULATES THE SECOND VIRIAL COEFFICIENT FOR HELIUM. THE RANGE IS FROM 2 TO 1500 DEG K. INPUT IS TEMPERATURE IN DEGREES KELVIN

UNITS ARE ATM, DEG KELVIN, AND MOLES/LITER, 4/3/69-1253, R. D. MCCARTY REVISED $2 / 12 / 70-925$

$1 B=0$. ODO

DO $5 \quad I=1,9$

$\mathrm{F} I=\mathrm{I}$

$5 B=B+T \star \star(1.5 D O-F I / 2 . D O) \star A(I)$

VI RB $=B$

RETURN

END

DOUBLE PRECISION FUNCTION DBDT ( T)

IMPLICIT REAL* $8(\mathrm{~A}-\mathrm{H})$

IMPLICIT REAL* $8(0-Z)$

I MPLICIT INTEGER*4( I-N)

DIMENSION A( 9), V( 45)

THIS SUB PROGRAM CALCULATES THE SECOND VIRIAL COEFFICIENT FOR HELIUM. THE RANGE IS FROM 2 TO 1500 DEG K. INPUT IS TEMPERATURE IN DEGREES KELVIN

DATA A / $-5.0815710041 \mathrm{D}-7,-1.1168680862 \mathrm{D}-4,1.1652480354 \mathrm{D}-2$,

$17.4474587998 \mathrm{D}-2,-5.3143174768 \mathrm{D}-1,-9.5759219306 \mathrm{D}-1$,

$23.9374414843 \mathrm{DO},-5.1370239224 \mathrm{DO}, 2$. $0804456338 \mathrm{DO} /$

$\mathrm{C}$

UNITS ARE ATM, DEG KELVIN, AND MOLES/LITER, 5/28/70-1630, R. D. MCCARTY

$1 B=0.0 D O$

DO $5 \mathrm{I}=1,9$

$\mathrm{F} I=\mathrm{I}$

$5 B=B+T \star \star(.5 D O-F I / 2 . D 0) \star A(I) \star(1.5 D 0-F I / 2 . D 0)$

$\mathrm{DBDT}=\mathrm{B}$

RETURN

END

DOUBLE PRECISION FUNCTION D2DBDT2( T)

IMPLICIT REAL*8 $(\mathrm{A}-\mathrm{H})$

IMPLICIT REAL*8 $(0-Z)$

IMPLICIT INTEGER*4(I-N)

DIMENSION A( 9)

DATA A / $-5.0815710041 \mathrm{D}-7,-1.1168680862 \mathrm{D}-4,1.1652480354 \mathrm{D}-2$,

$17.4474587998 \mathrm{D}-2,-5.3143174768 \mathrm{D}-1,-9.5759219306 \mathrm{D}-1$,

$23.9374414843 \mathrm{DO},-5.1370239224 \mathrm{DO}, 2$. $0804456338 \mathrm{DO} /$

$\mathrm{C}$

THIS SUB PROGRAM CALCULATES THE SECOND VIRIAL COEFFICIENT FOR HELIUM. THE RANGE IS FROM 2 TO 1500 DEG K. INPUT IS TEMPERATURE I N DEGREES KELVIN

$1 B=0.0 D O$

DO $5 \quad I=1,9$

$\mathrm{F} I=\mathrm{I}$

$5 B=B+T \star \star(.5 D O-F I / 2 . D O-1 . D 0) \star(1.5 D 0-F I / 2 . D 0) \star(.5 D O-F I / 2 . D O) \star A(I)$

$\mathrm{D} 2 \mathrm{DBDT} 2=\mathrm{B}$

RETURN

END

DOUBLE PRECISION FUNCTION FINDTV(PP) 


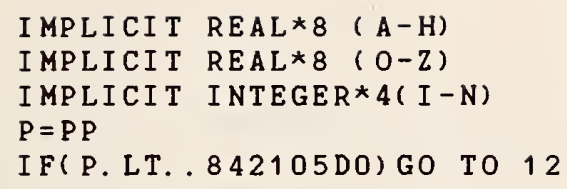

11 FINDTV $=$ T

RETURN

100 FORMAT(" TEMPERATURE ITTERATION FAILED AT T=', E14.7)

END

DOUBLE PRECISION FUNCTION DPDTVP(TT)

I MPLICIT REAL*8 ( A-H)

I MPLICIT REAL*8 $(0-Z)$

I MPLICIT INTEGER*4( I-N)

DIMENSION $C(12), D(14)$

DATA C/ - 3. $9394635287 \mathrm{DO}, 141.27497598 \mathrm{DO},-1640.7741565 \mathrm{DO}$

$1,11974.557102 \mathrm{DO},-55283.309818 \mathrm{DO}$,

$1166219.56504 \mathrm{DO},-325212.82840 \mathrm{DO}, 398843.22750 \mathrm{DO}$,

2-277718.06992D0,83395.204183DO, O. DO, O. DO/

DATA D $/-49.510540356 \mathrm{DO}, 651.9236417 \mathrm{DO},-3707.5430856 \mathrm{DO}$

$1,12880.673491 \mathrm{DO}$,

$1-30048.545554 \mathrm{DO}, 49532.267436 \mathrm{DO},-59337.558548 \mathrm{DO}, 52311.296025 \mathrm{DO}$,

$2-33950.233134 \mathrm{DO}, 16028.674003 \mathrm{DO},-5354.1038967 \mathrm{DO}, 1199.0301906 \mathrm{DO}$,

$3-161.46362959 \mathrm{DO}, 9.8811553386 \mathrm{DO} /$

$\mathrm{P}=0$. ODO

$\mathrm{T}=\mathrm{T} T-\mathrm{DELT}(\mathrm{TT})$

IF ( T - 2.1720DO) 10,10,1

1 DO $5 \quad I=1,10$

$5 P=P+C(I) \star T \star \star(1-I) \star(2-I)$

DPDTVP $=\mathrm{P} * V P N(T)$

RETURN

10 DO $15 I=1,14$

$15 P=P+D(I) * T \star \star(1-I) *(2-I)$

$D P D T V P=P \star V P N(T)$

RETURN

END

DOUBLE PRECISION FUNCTION VPN(TT)

I MPLICIT REAL* $8(\mathrm{~A}-\mathrm{H})$

IMPLICIT REAL*8 $(0-Z)$

I MPLICIT INTEGER* $4(\mathrm{I}-\mathrm{N})$

DI MENSION C ( 12$), D(14)$

DATA C/ $-3.9394635287,141.27497598,-1640.7741565,11974.557102$,

$1-55283.309818,166219.56504,-325212.82840,398843.22750$,

2-277718.06992,83395.204183,0.DO, O. DO/

DATA D / - 49. $510540356,651.9236417,-3707.5430856,12880.673491$,

$1-30048.545554,49532.267436,-59337.558548,52311.296025$,

$2-33950.233134,16028.674003,-5354.1038967,1199.0301906$,

$3-161.46362959,9.8811553386 /$

$\mathrm{T}=\mathrm{T} \mathrm{T}$

$\mathrm{T}=\mathrm{T}-\mathrm{DELT}(\mathrm{T})$

$\mathrm{P}=0.0 \mathrm{DO}$

I F $(\mathrm{T}-2.1720 \mathrm{DO}) 10,10,1$

1 DO $5 I=1,10$

$5 \mathrm{P}=\mathrm{P}+\mathrm{C}(\mathrm{I}) \star \mathrm{T} \star \star *(2-\mathrm{I})$ 
$V P N=D E X P(P) / .76 D+6$

RETURN

10 DO $15 \mathrm{I}=1,14$

$15 \mathrm{P}=\mathrm{P}+\mathrm{D}(\mathrm{I}) \star \mathrm{T} \star \star(2-\mathrm{I})$

$V P N=D E X P(P) / .76 D+6$

RETURN

END

DOUBLE PRECISION FUNCTION DELT(TT)

IMPLICIT REAL* $8(\mathrm{~A}-\mathrm{H})$

IMPLICIT REAL* $8(O-Z)$

IMPLICIT INTEGER*4(I-N)

$\mathrm{T}=\mathrm{T} \mathrm{T}$

DELT $=.001 \mathrm{DO}+.002 \mathrm{DO} * \mathrm{~T}$

RETURN

END

DOUBLE PRECISION FUNCTION SATV(TT)

IMPLICIT REAL*8 (A-H)

I MPLICIT REAL*8 $(0-\mathrm{Z})$

IMPLICIT INTEGER*4(I-N)

DIMENSION GV (6)

DATA GV/-.069267495322DO, -. $1292532553 \mathrm{DO}, .29347470712 \mathrm{DO}$

$1,-.40806658212 \mathrm{DO}, .35809505624 \mathrm{DO},-.11315580397 \mathrm{DO} /$

DATA DC/.06964DO/

DATA TC/5.2014 DO/

$\mathrm{T}=\mathrm{T} \mathrm{T}$

$D C A L=D C$

$\mathrm{R}=(1 . \mathrm{DO} / \mathrm{TC})$

DO $1 \quad I=1,6$

$F I=I$

$1 D C A L=D C A L+G V(I) \star R \star \star(E I / 3 . D O)$

$S A T V=D C A L$

RETURN

END

DOUBLE PRECISION FUNCTION SATL(TT)

IMPLICIT REAL*8 $(\mathrm{A}-\mathrm{H})$

I MPLICIT REAL* $8(0-Z)$

I MPLICIT INTEGER* $4(\mathrm{I}-\mathrm{N})$

DIMENSION GL( 9 )

DATA GL/. $12874326484 \mathrm{DO},-.43128217346 \mathrm{DO}, 1.7851911824 \mathrm{DO}$

$1,-3.3509624489 \mathrm{DO}, 3.0344215824 \mathrm{DO},-1.0981289602 \mathrm{DO}, \mathrm{O} . \mathrm{DO}, \mathrm{O} . \mathrm{DO}, \mathrm{O} . \mathrm{DO} /$

DATA DC/.06964DO/

DATA TC/5.2014D0/

$\mathrm{T}=\mathrm{T} \mathrm{T}$

$D C A L=D C$

$\mathrm{R}=(1 . \mathrm{DO}-\mathrm{T} / \mathrm{TC})$

DO $2 \mathrm{I}=1,6$

$\mathrm{FI}=\mathrm{I}$

$2 D C A L=D C A L+G L(I) \star R \star \star(E I / 3 . D O)$

$S A T L=D C A L$

RET URN

END

DOUBLE PRECISION FUNCTION SURFTEN( T)

I MPLICIT REAL* $8(\mathrm{~A}-\mathrm{H})$

IMPLICIT REAL*8 $(0-Z)$

IMPLICIT INTEGER*4( I - N )

SURFTEN $=.5308 \mathrm{DO} *(1 .-\mathrm{T} / 5.2014 \mathrm{DO})$

RETURN

END

DOUBLE PRECISION FUNCTION R INDEX( D, H)

IMPLICIT REAL* $8 \quad(\mathrm{~A}-\mathrm{H})$

I MPLICIT REAL*8 $(\mathrm{O}-\mathrm{Z})$

IMPLICIT INTEGER* $4(\mathrm{I}-\mathrm{N})$

$D D=D \star 4.0026 D 0 / 1000$. DO

$A L G=0.123396 D 0-0.0014 \star D D+33701.617944 / D O W \star \star 2-12325284955 . D 0 / W \star \star 4$

$F A C=A L G * D D / 0.95555 D 0$

$R=((2 . D O \star F A C+1 . D O) /(F A C-1 . D 0))$

$I F(R . L T, 0.0 D 0) R=R *(-1$.

$R=R \star \star(.5 D O)$ 
RI NDEX $=$ R

RETURN

END

DOUBLE PRECISION FUNCTION TMELT( PP)

IMPLICIT REAL* $8(\mathrm{~A}-\mathrm{H})$

I MPLICIT REAL $8(0-Z)$

I MPLICI T INTEGER* $4(\mathrm{I}-\mathrm{N})$

$P=P P \star 1.01325 \mathrm{DO} / .98066 \mathrm{D} 0$

I F ( P-17.8ODO. LT, O, ODO ) GO TO 1

TMELT $=((P-17.80 D 0) / 17.3145 \mathrm{DO}) \star \star(1 . / 1.555414 \mathrm{DO})$

RETURN

1 TMELT $=2$. DO

RETURN

END

DOUBLE PRECISION FUNCTION FDIEL(D)

I MPLICI T REAL* $8(\mathrm{~A}-\mathrm{H})$

IMPLICI T REAL* $8(0-Z)$

I MPLICIT IN $\approx E G E R \star 4(I-N)$

$\mathrm{DD}=\mathrm{D} \star 4.0026 \mathrm{D} 0 / 1000 . \mathrm{DO}$

$A L P H A=.123396 \mathrm{DO}-.0014 \mathrm{DO} \star \mathrm{DD}$

$A L P H A=A L P H A \star D D \star 1.04652 D 0$

$E P S=(1 . D O+2$. DO $A L P H A) /(1 . D O-A L P H A)$

FDIEL $=$ EPS

RETURN

END

DOUBLE PRECISION FUNCTION THERM( DD, TT)

IMPLICI T REAL*8 ( A-H)

I MPLICIT REAL*8 $(0-Z)$

IMPLICIT INTEGER* $4(\mathrm{I}-\mathrm{N})$

HELI UM

THIS ROUTINE CALCULTATES THERMAL CONDUCTIVITY AND VISCOSITY

FOR AN INPUT OF DEGREES KELVIN AND DENSITY IN MOLES PER LITER

THE RANGE OF TEMPERATURE IS FROM 2 TO $2000 \mathrm{~K}$

FOR TEMPERATURES BELOW 300 K FORMULAS OFD VINCE ARP AND GE STEWARD

ARE USED, FOR TEMPERATURES ABOVE 300 THE DILUTE GAS OF A CRITICAL

COMPILATION FROM ENGLAND IS USED FOR BOTH VISCOSITY AND

THERMAL CONDUCTIVITY AND THE EXCESS FUNCTIONS FROM THE ROUTINES BY ARP AND STEWART) THE EXCESS FUNCTIONS ARE CALCULATED FOR TEMPS

ABOVE $300 \mathrm{~K}$ WITH THE TEMPERATURE DEPENDENCE HELD AT $300 \mathrm{~K}$

FOR TEMPS BELOW $300 \mathrm{~K}$ TO $100 \mathrm{~K}$ THE VISCOSITY EXCESS IS CALC

FROM STEWARTS ROUTINE BUT THE DILUTE GAS VALUES ARE TAKEN FROM

THE ENGLISH CORRELATION FOR TEMPS BETWEEN 100 AND 110 T

DILUTE GAS CALCULATION IS AVARAGED

$1 \quad D=D D$

$\mathrm{T}=\mathrm{T} \mathrm{T}$

$\mathrm{RHO}=\mathrm{D} * 4.0026 \mathrm{D}-3$

IF(T. LT. 300. DO) GO TO 5

TH030 =VI SCX $(300$. DO $) \star .00781736$ DO

$\mathrm{THO} 300=\operatorname{CONZ}(300$. DO)

DEL $300=\mathrm{DELC}(300$. DO, RHO)

$\mathrm{THO}=\mathrm{VISCX}(\mathrm{T}) \star .00781736 \mathrm{DO}+\mathrm{THO}$ Н

THE $=$ THO300*DEL $300-$ THO 300

THE RM $=$ THO+ THE

RETURN

5 THERM $=\operatorname{CONZ}(\mathrm{T}) \star \mathrm{DELC}(\mathrm{T}, \mathrm{RHO})+\mathrm{CRITIC}(\mathrm{T}, \mathrm{RHO})$

OUTPUT IN MW/CM. K

RETURN

END

DOUBLE PRECISION FUNCTION VISC(DD, TT)

I MPLICI T REAL* $8(\mathrm{~A}-\mathrm{H})$

I MPLICIT REAL* $8(0-Z)$

IMPLICIT INTEGER* $4($ I $-N)$

$\mathrm{D}=\mathrm{DD}$

$\mathrm{T}=\mathrm{T} \mathrm{T}$

IF ( T. LT. 100. DO) GO TO 10

IF(T. LT. 300. DO) GO TO 8

ETAO=VISCX(T)

ET030 = VISCX( 300. DO $)$ 
ETO300 $=\operatorname{VISCDT}(0.0 D 0,300 . \mathrm{DO})$

ETE $300=V I \operatorname{SCDT}(D, 300 . D 0)-E T 0300$

$V I S C=E T A O+E T E 300$

$\mathrm{C}$

OUTPUT UNITS ARE MICROPOISE

RETURN

8 IF( T. LT. 110. DO) GO TO 9

$E T A O=V I S C X(T)$

$E T E B=V I S C D T(D, T)-V I \operatorname{SCDT}(0.0 D O, T)$

$V I S C=E T A O+E T E B$

RETURN

9 ETA1 $=$ VISCDT( 0. ODO, 100. DO)

$E T A 2=V I S C X(110 . D O)$

$E T A O=E T A 1+(E T A 2-E T A 1) \star(T-100 . D 0) / 10 . D O$

VI SC $=E T A O+V I S C D T(D, T)-V I S C D T(O . O D O, T)$

RET URN

$10 \mathrm{VISC}=\mathrm{VISCDT}(\mathrm{D}, \mathrm{T})$

RETURN

END

DOUBLE PRECISION FUNCTION VISCX( T )

I MPLICIT REAL* $8(\mathrm{~A}-\mathrm{H})$

IMPLICIT REAL* $8(0-Z)$

I MPLICI T INTEGER* $4($ I $-N$ )

VI SCX $=196 . \mathrm{DO} * \mathrm{~T} * \star .71938 \mathrm{DO} * \mathrm{DEXP}(12.451 \mathrm{DO} / \mathrm{T}-295.67 \mathrm{DO} / \mathrm{T} / \mathrm{T}-4.1249 \mathrm{DO})$ RETURN

END

DOUBLE PRECISION FUNCTION DELC(TEMP, RHO)

IMPLICIT REAL*8 (A-H)

I MPLICIT REAL*8 $(\mathrm{O}-\mathrm{Z})$

I MPLICIT INTEGER* $4($ I $-N)$

$\mathrm{C}$

$\mathrm{K}=\mathrm{KZERO} * \operatorname{EXPF}(\mathrm{B}(\mathrm{T}) \star \mathrm{RHO}+\mathrm{C}(\mathrm{T}) \star \mathrm{RHO} * \star 2)$

THIS PROGRAM RETURNS EXPF $(B(T) \star R H O+C(T) \star R H O \star \star 2)$

$1 \quad B B=D L O G(T E M P)$

$\mathrm{CC}=1$. DO $/ \mathrm{TEMP}$

$\mathrm{BETTY}=4.7470660612 \mathrm{D} 0-5.3641468153 \mathrm{DO} * \mathrm{BB}+3.4639703698 \mathrm{DO} * \mathrm{BB} * 2$

$2-1.0702455443 \mathrm{DO} * \mathrm{BB} * 3+0.1571349306 \mathrm{DO} * \mathrm{BB} * 44-.00892140047 \mathrm{DO} * \mathrm{BB} * 5$

$\mathrm{B}=\mathrm{DEXP}(\mathrm{BETTY})$

$\mathrm{C}=2.2109006708 \mathrm{D} 0+187.74174808 \mathrm{DO} * \mathrm{CC}-1281.0947055 \mathrm{D} 0 * \mathrm{CC} * \mathrm{CC}$

$3+3645.2393216 \mathrm{DO} * \mathrm{CC} \star 3 * 3-3986.6937948 \mathrm{DO} * \mathrm{CC} \star 4$

$\mathrm{DELC}=\mathrm{DEXP}(\mathrm{B} \star \mathrm{RHO}+\mathrm{C} \star \mathrm{RHO}$ * $\mathrm{RHO})$

RETURN

END

DOUBLE PRECISION FUNCTION CONZ(TEMP)

I MPLICIT REAL*8 $(\mathrm{A}-\mathrm{H})$

I MPLICI T REAL*8 $(0-Z)$

IMPLICIT INTEGER*4( I-N)

$\mathrm{C}$

KZERO IN MILLIHATTS/CM-K, T IN KELVIN9 22 JUNE 71.

$1 \quad$ ANNE $=$ DLOG ( TEMP)

$\mathrm{PAT}=-4.3611622157 \mathrm{DO}+1.9250159286 \mathrm{DO} * \mathrm{ANNE}-0.52544120165 \mathrm{DO}$ * ANNE**2

$1+.090045763885 \mathrm{DO} \star \mathrm{ANNE} \star * 3-.0054773874708 \mathrm{DO} * \mathrm{ANNE} \star * 4$

CONZ $=$ DEXP( PAT)

RETURN

END

DOUBLE PRECISION FUNCTION VISCDT( DGC, T)

I MPLICIT REAL*8 ( A-H)

IMPLICI T REAL*8 $(0-Z)$

IMPLICIT INTEGER* $4(\mathrm{I}-\mathrm{N})$

W. G. STEHARD, S DATA 23 JUNE 71

INPUT UNITS ARE KELVIN AND MOL/LITER

OUTPUT UNITS ARE MICROPOISE

$T L=D L O G(T)$

$\mathrm{R}=\mathrm{DGC} \star 4.0026 \mathrm{D} 0 / 1000 . \mathrm{DO}$

$\mathrm{ANNE}=-0.135311743 \mathrm{DO} / \mathrm{TL}+1.00347841 \mathrm{DO}+1.20654649 \mathrm{DO} * \mathrm{TL}$

$1-0.149564551 \mathrm{DO} \star \mathrm{TL} \star \mathrm{TL}+0.0125208416 \mathrm{DO} \star \mathrm{TL} \star \star 3$

$\mathrm{BETTY}=\mathrm{R} *(-47.5295259 \mathrm{DO} / \mathrm{TL}+87.6799309 \mathrm{DO}-42.0741589 \mathrm{DO} \star \mathrm{TL}$

$1+8.33128289 \mathrm{DO} * \mathrm{TL} * \mathrm{TL}-0.589252385 \mathrm{DO} * \mathrm{TL} \star \star 3)$

$\mathrm{CAROL}=\mathrm{R} * \mathrm{R} *(547.309267 \mathrm{D} 0 / \mathrm{TL}-904.870586 \mathrm{DO}+431.404928 \mathrm{DO} * \mathrm{TL}$

$1-81.4504854 \mathrm{DO} * \mathrm{TL} * \mathrm{TL}+5.37008433 \mathrm{DO} * \mathrm{TL} * * 3)$

$\mathrm{DAGMAR}=\mathrm{R} * 3 *(-1684.39324 \mathrm{DO} / \mathrm{TL}+3331.08630 \mathrm{DO}-1632.19172 \mathrm{DO} * \mathrm{TL}$ 


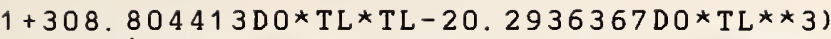

VISCDT $=D E X P(A N N E+B E T T Y+C A R O L+D A G M A R)$

RETURN

END

DOUBLE PRECISION FUNCTION CRITIC( TEMP, RHO)

IMPLICIT REAL* $8(\mathrm{~A}-\mathrm{H})$

IMPLICIT REAL*8 $(0-Z)$

IMPLICIT INTEGER*4( I-N)

C CRITICAL ANOMALY FOR HE THERM. CON., SCALED FROM H-2

C T IN KELVIN, REQUIRES DENSITY IN GRAMS/CC AND CP IN JOULES/MOLE

C

THIS DECK OF 18 SEPT 70, I HAVE USED MCCARTY"S HE DECKS OF $7 / 18 / 70$

$4 \mathrm{~T}=\mathrm{TEMP}$

5 DML $=$ RHO $/ 0.0040026 \mathrm{DO}$

6 IF( T. GE. 11.83DO) GO TO 11

IF( RHO. GT. 0.12DO) GO TO 11

$7 \quad C P 1=C P(D M L, T)$

$8 \quad C P 2=C P(D M L, 11.83 D 0)$

9 CRITIC $=0.0026 \mathrm{DO} *(\mathrm{CP} 1-\mathrm{CP} 2) / 4.0026 \mathrm{D} 0$

10 IF( CRI TIC) $11,12,12$

11 CRI TIC $=0$. ODO

12 RETURN

END

HE L I UM. COF

$-.1509686261900 E-06$

$.6464089890400 \mathrm{E}-06$

$.4136235736700 E-04$

-. $3791019035300 E-03$

. $1380645404900 E-02$

-. $2508541205800 E-02$

. $2369756039800 E-02$

-. $9572646106600 \mathrm{E}-03$

. $3740593182800 \mathrm{E}-04$

$-.6410322033300 E-03$

$.1857936617700 \mathrm{E}-02$

$7400798660600 E-03$

$1479256814800 E-03$

-. $3253135547700 E-02$

$1951873928600 \mathrm{E}-01$

-. $1057181713500 \mathrm{E}+00$ $3316494444900 E+00$

-. $5113002253500 E+00$ $3994000490600 E+00$

-. $1555524447100 \mathrm{E}+00$

$.4906264031000 E-02$

-. $2614800437700 E-01$

. $3422168554500 \mathrm{E}-01$

$5415966262200 E-05$

-. $1068780677700 \mathrm{E}-04$

-. $8948465186900 \mathrm{E}-05$

-. $2500000000000 \mathrm{E}-02$

-. $4228745462600 \mathrm{E}-07$

$4452935441300 E-06$

- $1024615095400 E-04$

$8525460895600 \mathrm{E}-04$

-.2516306925500E-03

$3287770928500 \mathrm{E}-03$

$-.1060195758000 \mathrm{E}-03$

$-.1068773807400 \mathrm{E}-03$ 
-. $3212095063200 E-04$ $1415901897000 E-03$ $1472563070100 E-02$

-. $2618354941000 \mathrm{E}-02$ $2046150111700 E-04$

. $1274699628800 E-02$

-. $2027292958300 \mathrm{E}-01$

. $7464803661500 \mathrm{E}-01$

-. $1721796652100 \mathrm{E}+00$

$.5105343973800 E+00$

-. $4017820269700 \mathrm{E}+00$

. $2682986463200 E+00$ $7906601204000 E-02$

-. $8939348565600 \mathrm{E}-01$

-. $1507658005300 \mathrm{E}+00$ $.2688249432700 E-05$

-. $3379431683500 E-04$

-. $2449595119500 E-04$

-. $5000000000000 E-03$

-. $1480219534800 \mathrm{E}-07$ $4172179111900 \mathrm{E}-06$

-. $2332655327100 E-06$ $4085511088000 \mathrm{E}-06$ $1090056796400 E-04$

-. $5006095277500 \mathrm{E}-04$ . $1131276504300 \mathrm{E}-03$

-. $1253984328700 \mathrm{E}-03$

. $1966138068800 \mathrm{E}-05$

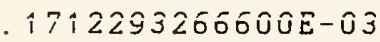

. $2305100056300 E-03$

-. $9656473910000 \mathrm{E}-03$

-. $3602773529200 \mathrm{E}-04$

. $1607994655500 E-02$

-. $2744176361500 E-01$

. $1473950695700 \mathrm{E}+00$

-. $4355934483800 E+00$

. $1344795607800 \mathrm{E}+01$

-. $1704037512500 E+01$ $9026267404000 E+00$

. $5687564411100 E-02$

-. $1443814662500 E+00$ $3376887485100 \mathrm{E}-02$ $1075420121800 E-05$

-. $4526462230800 E-04$ $3859738886400 E-04$

-. $5000000000000 E-03$ 1500 .

2. $17 D 0 \quad 100$.

5. 2014

17. 399

.22746 
NBS-114A (REV. 2.8C)

U.S. DEPT. OF COMM.

BIBLIOGRAPHIC DATA

SHEET (See in struction s)

4. TITLE AND SUBTITLE

INTERACTIVE FORTRAN PROGRAMS FOR MICRO COMPUTERS TO CALCULATE THE THERMOPHYSICAL PROPERTIES OF TWELVE FLUIDS [MIPROPS]
PUBLICATION OR REPORT NO.

NBS/ TN-1097
2. Performing Organ. Report No.

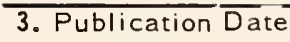

May 1986

\section{5. $A \cup T H O R(S)$}

Robert D. McCarty

6. PERFORMING ORGANIZATION (If joint or other than NBS, see instructions)

\section{NATIONAL BUREAU OF STANDARDS}

DEPARTMENT OF COMMERCE

WASHINGTON, D.C. 20234

9. SPONSORING ORGANHZATHOIH IAAME AHSD COMPLETE ADCRESS (Strat, City, Stute, ZHF)

National Aeronautics and Space Administration

Lyndon B. Johnson Space Center

Houston, Texas 77058

10. SUPPLEMENTARY NOTES

[Document describes a computer program; SF-185, FIPS Software Summary, is attached.

11. ABSTRACT (A 200-word or less factual summary of most significant information. If document includes a significant bibliography or literature survey, mention it here)

The thermophysical and transport properties of selected fluids have been programmed in FORTRAN 77 which is available for micro computers. The input variables are any two of P, p, T (pressure, density, and temperature) in the single phase regions, and either $\mathrm{P}$ or $\mathrm{T}$ for the saturated liquid or vapor states. The output is pressure, density, temperature, internal energy, enthalpy, entropy, specific heat capacities $\left(C\right.$ and $C_{V}$ ), speed of sound and, in most cases, viscosity, thermal conductivity, and dielectric constant.

The fluids included are: helium, hydrogen, nitrogen, oxygen, argon, nitrogen trifluoride, methane, ethylene, ethane, propane, iso- and normal butane. The programs give properties in both the liquid and vapor states over a wide range of temperature and pressure. Copies of the program may be obtained from the office of Standard Reference Data, Room A320, Physics Building, National Bureau of Standards, Gaithersburg, MD 20899.

12. KEY WORDS (Six to twelve entries; alphabetical order; capitalize only proper names; and separate key words by semicolons) argon; computer programs; density; dielectric constant; enthalpy; entropy; equation of state; ethane; ethylene; heat capacity; helium; hydrogen; internal energy; isobutane; methane; nitrogen; nitrogen trifluoride; normal butane; oxygen; pressure; speed of sound; temperature; thermal conductivity; viscosity.

13. AVAILABILITY

XXUnlimited

$\square$ For Official Distribution. Do Not Release to NTIS

XXYrder From Superintendent of Documents, U.S. Government Printing Office, Washington, D.C. 20402.

$\square$ Order From National Technical Information Service (NTIS), Springfield, VA. 2216I
14. NO. OF PRINTED PAGES

$$
90
$$

15. Price 





\section{Technical Publications}

\section{Periodical}

Journal of Research-The Journal of Research of the National Bureau of Standards reports NBS research and development in those disciplines of the physical and engineering sciences in which the Bureau is active. These include physics, chemistry, engineering, mathematics, and computer sciences. Papers cover a broad range of subjects, with major emphasis on measurement methodology and the basic technology underlying standardization. Also included from time to time are survey articles on topics closely related to the Bureau's technical and scientific programs. Issued six times a year.

\section{Nonperiodicals}

Monographs-Major contributions to the technical literature on various subjects related to the Bureau's scientific and technical activities.

Handbooks-Recommended codes of engineering and industrial practice (including safety codes) developed in cooperation with interested industries, professional organizations, and regulatory bodies.

Special Publications-Include proceedings of conferences sponsored by NBS, NBS annual reports, and other special publications appropriate to this grouping such as wall charts, pocket cards, and bibliographies.

Applied Mathematics Series-Mathematical tables, manuals, and studies of special interest to physicists, engineers, chemists, biologists, mathematicians, computer programmers, and others engaged in scientific and technical work.

National Standard Reference Data Series-Provides quantitative data on the physical and chemical properties of materials, compiled from the world's literature and critically evaluated. Developed under a worldwide program coordinated by NBS under the authority of the National Standard Data Act (Public Law 90-396).

NOTE: The Journal of Physical and Chemical Reference Data (JPCRD) is published quarterly for NBS by the American Chemical Society (ACS) and the American Institute of Physics (AIP). Subscriptions, reprints, and supplements are available from ACS, 1155 Sixteenth St., NW, Washington, DC 20056.

Building Science Series-Disseminates technical information developed at the Bureau on building materials, components, systems, and whole structures. The series presents research results, test methods, and performance criteria related to the structural and environmental functions and the durability and safety characteristics of building elements and systems.

Technical Notes-Studies or reports which are complete in themselves but restrictive in their treatment of a subject. Analogous to monographs but not so comprehensive in scope or definitive in treatment of the subject area. Often serve as a vehicle for final reports of work performed at NBS under the sponsorship of other government agencies.

Voluntary Product Standards-Developed under procedures published by the Department of Commerce in Part 10, Title 15, of the Code of Federal Regulations. The standards establish nationally recognized requirements for products, and provide all concerned interests with a basis for common understanding of the characteristics of the products. NBS administers this program as a supplement to the activities of the private sector standardizing organizations.

Consumer Information Series-Practical information, based on NBS research and experience, covering areas of interest to the consumer. Easily understandable language and illustrations provide useful background knowledge for shopping in today's technological marketplace.

Order the above NBS publications from: Superintendent of Documents, Government Printing Office, Washington, DC 20402.

Order the following NBS publications-FIPS and NBSIR's-from the National Technical Information Service, Springfield, VA 22161.

Federal Information Processing Standards Publications (FIPS PUB)-Publications in this series collectively constitute the Federal Information Processing Standards Register. The Register serves as the official source of information in the Federal Government regarding standards issued by NBS pursuant to the Federal Property and Administrative Services Act of 1949 as amended, Public Law 89-306 (79 Stat. 1127), and as implemented by Executive Order 11717 (38 FR 12315, dated May 11, 1973) and Part 6 of Title 15 CFR (Code of Federal Regulations).

NBS Interagency Reports (NBSIR)-A special series of interim or final reports on work performed by NBS for outside sponsors (both government and non-government). In general, initial distribution is handled by the sponsor; public distribution is by the National Technical Information Service, Springfield, VA 22161, in paper copy or microfiche form. 
U.S. Department of Commerce National Bureau of Standards

Gaithersburg, MD 20899

Official Business

Penalty for Private Use $\$ 300$

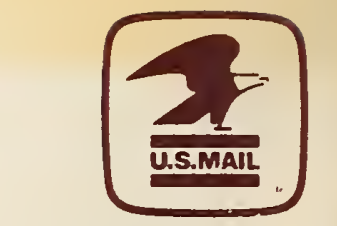

POSTAGE AND FEES PAID U.S. DEPARTMENT OF COMMERCE COM-215

SPECIAL FOURTH-CLASS RATE BOOK 